\title{
The Pulse of Asia
}

April 19-20, 2013, Seoul, Korea

Guest Editor

Jeong Bae Park, Seoul

\section{Contents}

\section{Best Orals}

36 Abstracts B-01-B-05

Moderated Posters

38 Abstracts M-01-M-77 


\section{Best Orals}

\section{B-01 \\ Derivation and Validation of Diagnostic Thresholds for Central Blood Pressure Measurements Based on Long-Term Cardiovascular Risks}

*Hao-Min Cheng 1,2,4,5, *Shao-Yuan Chuang ${ }^{7}$, Shih-Hsien Sung 3,4,6, Wen-Chung Yu ${ }^{3,4,5}$, Alan Pearson ${ }^{7}$, Edward G. Lakatta ${ }^{8}$, *Wen-Harn Pan ${ }^{7}$, *Chen-Huan Chen ${ }^{2,4,5,6}$

*These Authors contributed equally to the present work; * Shao-Yuan Chuang is a co-first author and Wen-Harn Pan is a co-corresponding author.

${ }^{1}$ The Joanna Briggs Institute, Faculty of Health Sciences, The University of Adelaide, Adelaide, Australia; ${ }^{2}$ Department of Medical Research and Education, and ${ }^{3}$ Department of Medicine, Taipei Veterans General Hospital, Taipei, Taiwan; ${ }^{4}$ Cardiovascular Research Center, ${ }^{5}$ Department of Medicine, and ${ }^{6}$ Institute of Public Health and Community Medicine Research Center, National Yang-Ming University, Taipei, Taiwan; ${ }^{7}$ Division of Preventive Medicine and Health Service, Research Institute of Population Health Sciences, National Health Research Institutes, Miaoli, Taiwan; ${ }^{8}$ The Laboratory of Cardiovascular Science in the National Institute on Aging Intramural Research Program in Baltimore, MD, USA

Background: Current guidelines for managing patients with hypertension mainly rely on blood pressure (BP) measured at brachial arteries (cuff BP). However, BP measured at the central aorta (central BP; CBP) may be a better prognostic factor for predicting future $\mathrm{CV}$ events than cuff BP. We derived and validated outcome-driven thresholds for CBP in two independent cohorts with long-term follow-up.

Method and Result: In a Derivation Cohort (1272 individuals and a median follow-up of 17 years), we determined the diagnostic thresholds for CBP by using current guidelineendorsed cutoffs for cuff BP with a bootstrap approach and an approximation method. To evaluate the discriminatory power in predicting cardiovascular outcomes, the derived thresholds were subsequently tested in a Validation Cohort (2501 individuals and a median follow-up of 13 years).

The two analyses yielded similar diagnostic thresholds for CBP. After rounding, systolic/diastolic threshold was $110 / 80 \mathrm{mmHg}$ for optimal BP and 130/90 $\mathrm{mmHg}$ for hypertension. Comparing to optimal BP, the risk of cardio- vascular mortality increased significantly in subjects with hypertension (hazard ratio 3.08, 95\% confidence interval 1.05-9.05). Of the multivariate Cox proportional-hazards model, incorporation of a dichotomous variable by defining hypertension as CBP $\geq 130 / 90 \mathrm{mmHg}$ was associated with the largest contribution to the predictive power.

Conclusion: CBP of $130 / 90 \mathrm{mmHg}$ was determined to be the cutoff limit for normality and was characterized by a greater discriminatory power for long-term events in our Validation Cohort. This report represents an important step toward the application of the CBP concept in clinical practice.

\section{B-02 \\ Four-Limb Blood Pressure as Predictors of Mortality in Elderly Chinese \\ Chang-Sheng Sheng, Ming Liu, Wei-Fang Zeng, Qi-Fang Huang, Yan Li, Ji-Guang Wang \\ Centre for Epidemiological Studies and Clinical Trials, The Shanghai Institute of Hypertension, Ruijin Hospital, Shanghai Jiao Tong University School of Medicine, Shanghai, China}

Background: The predictive value of blood pressure for cardiovascular morbidity and mortality diminishes in the elderly, which may be confounded and compensated by the blood pressure differences across the four limbs, markers of peripheral arterial disease.

Methods: In a prospective elderly ( $\geq 60$ years) Chinese study. we performed simultaneous four-limb blood pressure measurement using an oscillometric device in the supine position, and calculated blood pressure differences between the four limbs.

Results: At baseline, the mean age of the 3133 participants (1383 men) was 69 years. During 4 years (median) of follow-up, all-cause and cardiovascular deaths occurred in 203 and 93 subjects, respectively. In multiple regression analyses, arm blood pressures on the higher arm side of systolic blood pressure did not predict mortality $(\mathrm{P} \geq 0.06)$ except for a negative association between mean arterial pressure and total mortality $(\mathrm{P}=0.04)$. However, in adjusted analyses, the hazard ratios associated with a 1-standard deviation decrease in ankle-brachial blood pressure index or increase in interarm or inter-ankle blood pressure difference were 1.15 to 1.23 for total mortality $(\mathrm{P} \leq 0.01)$ and 1.17 to 1.24 for cardiovascular mortality $(P \leq 0.04)$. In categorical analyses, similar results were observed for a decreased ankle-brachial index ( $\leq$ $0.90, \leq 0.95$ or $\leq 1.00$ ) or increased inter-arm or inter-ankle difference ( $\geq 15 \mathrm{~mm} \mathrm{Hg}$ or $\geq 10 \mathrm{~mm} \mathrm{Hg}$ ). 
Conclusion: In the elderly, above and beyond arm blood pressure level and together with ankle-brachial index, the inter-arm and inter-ankle blood pressure differences improve prediction of mortality. Simultaneous four-limb blood pressure measurement has become feasible with current technology and might be useful in cardiovascular prevention.

\section{B-03 \\ Arterial Stiffness and Wave Reflections in Young and Older Patients with Isolated Diastolic Hypertension}

\section{Fang-Fei Wei, Lu Zhang, Ting-Yan Xu, Yan Li}

From Center for Vascular Evaluations, Shanghai Key Lab of Hypertension, Shanghai Institute of Hypertension, Ruijin Hospital, Shanghai Jiao Tong University School of Medicine, Shanghai 200025, China

Background: Isolated systolic hypertension (ISH) is characterized by stiff artery in the elderly and large pressure amplification in the youth. While little is known about the arterial change associated with isolated diastolic hypertension (IDH).

Methods: A total of 1102 consecutive untreated outpatients in our Hypertension Clinic were investigated. Systolic phase and diastolic phase hypertension was respectively defined as a 24-hour average blood pressure of at least 130 $\mathrm{mm} \mathrm{Hg}$ systolic and $80 \mathrm{~mm} \mathrm{Hg}$ diastolic. Patients were diagnosed as normotension, IDH, ISH or systolic and diastolic biphase hypertension (SDH) according to the presence of diastolic or systolic hypertension. Carotid-femoral pulse wave velocity (cfPWV), and central augmentation index (CAIx) were determined by the SphygmoCor system. We applied analysis of variance to compare the arterial parameters between groups while accounting for age, sex, body mass index, current tobacco and alcohol consumption, 24-hour pulse rate, serum total cholesterol and blood glucose.

Results: IDH was diagnosed in 68, 186 and 31 patients in young (19 40 years), middle-aged (41 60 years) and older (60 81 years) groups, respectively. The corresponding values were 2, 19 and 23 for ISH and 81, 234 and 40 for SDH, respectively. Patients with IDH had a significantly higher CAIx compared with normotensives in young (13.8 vs. 8.3\%, $P=$ $0.0091)$ and middle-aged (26.3 vs. $24.2 \%, P=0.013$ ) group, but not in older group ( 30.2 vs. $27.8 \%, P=0.20$ ). In all three age groups, IDH patients had a smilar cfPWV $(P>0.11)$ as normotensives. Compared with normotensives, patients with ISH had an increased cfPWV in middle-aged ( 8.5 vs $7.5 \mathrm{~m} / \mathrm{s}$, $P<0.001)$ and older ( 9.2 vs $8.3 \mathrm{~m} / \mathrm{s}, P=0.009)$ groups, and patients with SDH had both elevated $(P<0.001)$ cfPWV and CAIx in young and middle-aged groups.

Conclusion: IDH was characterized by an increased systolic augmentation and a normal large arterial stiffness in young and middle-aged subjects. It indicated that IDH might be mainly due to the disturbance in small arteries.

\section{B-04}

The Associations of N-Terminal Pro B-Type Natriuretic Peptide with Day-by-Day Blood Pressure or Heart Rate Variability in a General Population: The Ohasama Study

Michihiro Satoh', Takayoshi Ohkubo², Miki Hosaka?, Takuo Hirose ${ }^{1,3}$, Ryusuke Inoue ${ }^{4}$, Hirohito Metoki ${ }^{5}$, Kei Asayama 1,6, Masahiro Kikuya ${ }^{5}, K^{1}$ oichi Node ${ }^{7}$, Yutaka Imai

${ }^{1}$ Department of Planning for Drug Development and Clinical Evaluation, Tohoku University Graduate school of Pharmaceutical Sciences, Sendai, Japan; ${ }^{2}$ Department of Health Science, Shiga University of Medical Science, Otsu, Japan; ${ }^{3}$ Early Development and Pathologies, Center for Interdisciplinary Research in Biology, Collège de France, Paris, France; ${ }^{4}$ Medical Information Technology Center, Tohoku University Hospital; ${ }^{5}$ Tohoku Medical Megabank Organization, Tohoku University, Sendai, Japan; ${ }^{6}$ Division of Hypertension and Cardiovascular Rehabilitation, Department of Cardiovascular Diseases, University of Leuven, Leuven, Belgium; ${ }^{7}$ Department of Cardiovascular Medicine, Saga University, Saga, Japan

Background: N-terminal pro B-type natriuretic peptide (NT-proBNP) is known to be a useful marker of myocardial damage or stress. Although previous studies reported NT-proBNP, day-by-day blood pressure (BP) and heart rate (HR) variabilities as cardiovascular risk factors, the associations of NT-proBNP with day-by-day BP and HR variabilities are still unknown.

Methods: We obtained NT-proBNP for 664 participants aged $\geq 35$ years with no past history of ischemic heart disease in the general population of Ohasama. We investigated the associations of NT-proBNP with day-by-day BP and HR variabilities by multiple regression analyses after adjusting for possible confounding factors including home BP hypertension ( $\geq 135 / 85 \mathrm{mmHg}$ or antihypertensive treatment). The day-by-day home BP and HR variabilities were calculated as within-participant standard deviation (SD) of the home BP and HR measurements, respectively.

Results: The 664 participants were $61.9 \pm 10.4$ years old, and included 468 (70.5\%) women and 250 (37.7\%) home BP hypertensives. The mean day-by-day systolic / diastolic $\mathrm{BP}$ and HR variabilities were $8.3 \pm 2.8 / 5.7 \pm 2.0 \mathrm{mmHg}$ and $5.3 \pm 2.0 \mathrm{bpm}$, respectively. The median Nt-proBNP (25th to 75th percentiles) was 44.9 (26.7 to 82.2 ) pg/mL. Participants with home BP hypertension had a higher Nt-proBNP than those with normotensives (57.1 vs. $39.7, \mathrm{P}=0.02$ ). The natural log-transformed (ln) Nt-proBNP was positively and significantly associated with day-by-day BP variability (regression coefficient per $1 \mathrm{SD}$ increase in InNTproBNP [ $\beta$ ] for systolic: $0.11, \mathrm{P}=0.0007, \beta$ for diastolic:0.09, $\mathrm{P}=0.005$ ). A significant association of $\operatorname{lnNt}$-proBNP with day-by-day HR variability was also observed after further adjusting for home HR levels ( $\beta=0.09, P=0.003)$. 
Conclusion: This study may raise the hypothesis that high day-by-day BP variability or HR variability may be associated with an elevated NT-pro BNP.

\section{B-05 \\ A Pharmacoepidemiologic Study of the Effects of Fimasartan on Cardiovascular Events and Metabolic Syndrome in Patients with Hypertension}

\author{
Changsoo Kim ${ }^{1,2}$, Dae Ryong Kang ${ }^{2}$, Min Young Kim ${ }^{3}$, \\ Jeong Bae Park ${ }^{3}$ \\ ${ }^{1}$ Division of Preventive Medicine, Department of \\ Medicine, Brigham and Women's Hospital, Boston, \\ MA, USA; ${ }^{2}$ Department of Preventive Medicine, \\ Yonsei University College of Medicine, Seoul, Korea; \\ ${ }^{3}$ Department of Medicine/Cardiology, Cheil General \\ Hospital, Kwandong University, Seoul, Korea
}

Background: A fimasartan, the 8th ARB, which was launched in March 2011, showed an excellent effects and good safety in large population in our previous cross-sectional study (Safe-Kanarb Study). But there is no long-term study to evaluate its efficacy on the major cardiovascular adverse events (MACE) and additional effects. The purpose of this study is to evaluate whether the early reduction of blood pressure (BP) and/or correction of metabolic derangement with fimasartan will affect differently the MACE and diabetes development long after in patients with hypertension (K-MetS study) after 3 years of follow-up.

Methods: The patients $(11,045)$ who were diagnosed as hypertension between October 2011 and October 2012 in Korea (700 private clinics and 11 university hospitals) were recruited. Among patients, 10,515 patients were enrolled and treated with fimasartan at a daily dose of 30-120 mg. Home blood pressure (BP) monitoring was performed in $2 / 3$ of patients in addition to clinic BP. Incidence of MACE (cardiovascular mortality, stroke, myocardial infarction, and hospitalization for heart failure) and the development of diabetes will be estimated after 3, 6, 12, 24, 36 months of follow-up. Baseline assessments including health behavior questionnaire, blood sampling, EKG and blood pressure measurement were conducted prior to fimarsartan treatment and will be repeated after 3, 6, 12 months.

Results: Among 10,514, 48.5\% were women. The mean age ( \pm Standard deviation) was $52.3( \pm 10.9)$ years old. The mean clinic systolic/diastolic BP was 144.8 $\pm 17.0 / 88.7 \pm 11.4$ $\mathrm{mmHg}$ at baseline, and mean home clinic systolic/diastolic BPs was $134.2 \pm 18.0 / 80.2 \pm 11.4 \mathrm{mmHg}$. The proportion of MetS at baseline was 52.3\%; presence of abdominal obesity (51.3\%), elevated fasting glucose (43.9\%), hypertriglyceride $(41.6 \%)$, and low high-density lipoprotein cholesterol (31.0\%). The proportion of complicated hypertension with diabetes, ischemic heart disease, stroke, heart failure and atrial fibrillation was $15.1,3.3,1.0,0.7$, and $0.4 \%$, respectively.
Conclusion: The K-MetS study is expected to provide valuable information about the effects of early BP control and correction of metabolic abnormality on the future cardiovascular outcome relatively in low-risk hypertension in a community.

\section{Moderated Posters}

\section{M-01 \\ Abnormal Deformation of Endothelial Cells in Atherosclerotic Blood Vessels}

Eunseok Seo, Kyung Won Seo, Sang Joon Lee

Pohang University of Science and Technology

Background: In vivo and in vitro experimental models for investigating the basic biophysics of atherosclerotic diseases were developed. In this study, the effects of extracellular matrix (ECM) on the thickness and structural variations of endothelial cells via collagenase treatment were experimentally investigated.

Methods: Zebrafishes fed with a normal diet and a cholesterol-enriched diet for four months were used in in vivo experiment. The morphological variations of endothelial cells and collagen fibers were observed using a transmission electron microscopy. In vitro models of human umbilical vein endothelial cells (HUVECs) were established on collagen films to mimic the experimental results obtained from in vivo study. Normal collagen films simulate the ECM in the blood vessels of a normal zebrafish, and the collagenase-treated (denatured) collagen films mimic the ECM in the blood vessels of an abnormal zebrafish. The cell growth of HUVECs on the ECM of the normal and abnormal blood vessels was monitored using a phase-contrast digital holographic microscopy.

Results: The endothelial cells of the zebrafish fed with a normal diet are tightly attached and aligned in good order. Their collagen layer has a fiber-like structure. By contrast, the endothelial cells of the zebrafish fed with high cholesterol diet are irregularly disorganized and their collagen has a broken structure. The HUVECs cultured on the collagen film are tightly attached, similar to those of a normal zebrafish. In addition, the cells are nearly uniformly distributed in a monolayer and they have similar shapes. However, the cells cultured on the degraded collagen film exhibit abnormal cell growth, such as cell aggregation and cluster formation.

Conclusion: The mechanical and chemical properties of the ECM seem to be closely related to endothelial dysfunction which is one of the main pathogenic factors of atherosclerosis. Better understanding on the outbreak mechanism of atherosclerosis would contribute to the design of novel therapies for clinical treatments of atherosclerosis. 


\section{M-02 \\ Cystein-Rich Angiogenic Protein 61 Promotes Vascular Smooth Muscle Cell Calcification via Metalloproteinase Activation}

\author{
Hae-Young Lee, Yeon-Yee Yoon, Byung-Hee Oh, \\ Hyo-Soo Kim \\ Dept of Internal Medicine, Seoul National University \\ College of Medicine
}

Background: Cystein-rich angiogenic protein 61 (CYR61, CCN1) was reported to be regulated by angiotensin II in vascular smooth muscle cells (SMCs) of atheromatous plaque. Because CYR61 was reported to induce osteoblastic differentiation of mesenchymal stem cells, we hypothesized that the CYR61 may play a role in vascular calcification.

Methods and Results: CYR61 expression was induced by 20.3 folds in SMCs harvested from thoracic aortas of male C57BL6 mouse after 16 hours of adenoviral vector (Ad-CYR61, $50 \mathrm{MOI}$ ) transfection, which significantly induced SMC calcification by $209.5 \pm 54.8 \%$ evaluated by Von Kossa staining after 14 days. In order to evaluate the full range of effects of CYR61 on SMC calcification, we performed microarray analysis. Several metalloproteinases such as MMP-13, -3, -10, and -8 were induced by $55.3,49.9,6.4$ and 5.2 folds, whereas tissue inhibitor of metalloproteinases such as TIMP-3 and -2 were reduced by $52 \%$ and $31 \%$ after 16 hours of Ad-CYR61 transfection, respectively (all p < 0.05). Remarkably, we found overall suppression of procollagen gene expression. Real time PCR confirmed MMP-13 gene induction by $33 \pm 13$ folds compared with control adenovirus transfected SMCs $(\mathrm{p}<0.05)$. Although mRNA or protein expressions of MMP-9 were not found increased, gelatin zymography showed an increased enzymatic activity of MMP-9 (92kDa) by $202 \pm 41 \%$ by Ad-CYR61, which was completely reversed by MMP-13 siRNA. Inhibition of MMP activity by the global MMP inhibitor, doxycycline completely blocked Ad-CYR61-induced calcification to the $87.0 \pm 30.8 \%$ of the control adenovirus transfected SMCs. Also, Ad-CYR61 failed to induce calcification in VSMCs harvested from MMP-9 knock out mouse (100.9 \pm $7.6 \%$ of the control adenovirus transfected SMCs, $\mathrm{p}=0.90$ ).

Conclusion: These findings demonstrate that CYR61 induces SMC calcification through MMP-13 - MMP-9 cascade. Therapies targeting this signaling pathway may regulate vascular calcification.

\section{M-03 \\ Vascular Calcifying Progenitor Cells Possess Bidirectional Differentiation Potentials and Regulate Ectopic and Atherosclerotic Calcification}

Hyun-Ju Cho, Hyun-Jai Cho, Ho-Jae Lee, Myung-Kang Song, Ji-Yun Seo, Yeon-Hee Bae, Ju-Young Kim, Hae-Young Lee, Whal Lee, Bon-Kwon Koo, Hyo-Soo Kim, Young-Bae Park, Byung-Hee Oh

Division of Cardiology, Department of Internal Medicine Seoul National University Hospital

Background: Vascular calcification is an advanced feature of atherosclerosis for which no effective therapy is available. To investigate the modulation or reversal of calcification, we identified calcifying progenitor cells and investigated their calcifying/decalcifying potentials.

Methods and Results: Cells from the aortas of mice were sorted into four groups using Sca- 1 and PDGFR $\alpha$ markers. Sca-1+ (Sca-1+/PDGFR $\alpha+$ and Sca-1+/PDGFR $\alpha$-) progenitor cells exhibited greater osteoblastic differentiation potentials than Sca-1- (Sca-1-/PDGFR $\alpha+$ and Sca-1-/PDGFR $\alpha-$ ) progenitor cells. Among Sca-1+ progenitor populations, Sca-1+/PDGFR $\alpha$ - cells possessed bidirectional differentiation potentials towards both osteoblastic and osteoclastic lineages, whereas Sca-1+/PDGFR $\alpha+$ cells differentiated into an osteoblastic lineage unidirectionally. When treated with a peroxisome proliferator activated receptor $\gamma$ (PPAR $\gamma$ ) agonist, Sca-1+/PDGFR $\alpha$ - cells preferentially differentiated into osteoclast-like cells. Sca-1+ progenitor cells in the artery originated from the bone marrow (BM) and could be clonally expanded. Vessel-resident BM-derived Sca-1+ calcifying progenitor cells displayed non-hematopoietic, mesenchymal characteristics. To evaluate the modulation of in vivo calcification, we established models of ectopic and atherosclerotic calcification. Computed tomography indicated that Sca-1+ progenitor cells increased the volume and calcium scores of ectopic calcification. However, Sca-1+/PDGFR $\alpha$ - cells treated with a PPAR $\gamma$ agonist decreased bone formation 2 -fold compared with untreated cells. Systemic infusion of Sca-1+/ PDGFR $\alpha$ - cells into Apoe-/- mice increased the severity of calcified atherosclerotic plaques. However, Sca-1+/PDGFR $\alpha-$ cells in which PPAR $\gamma$ was activated displayed markedly decreased plaque severity. Immunofluorescent staining indicated that Sca-1+/PDGFR $\alpha$ - cells mainly expressed osteocalcin; however, activation of PPAR $\gamma$ triggered receptor activator for nuclear factor- $\mathrm{\kappa B}$ (RANK) expression, indicating their bidirectional fate in vivo.

Conclusion: These findings suggest that a subtype of BM-derived and vessel-resident progenitor cells offer a therapeutic target for the prevention of vascular calcification and that PPAR $\gamma$ activation may be an option to reverse calcification. 


\section{M-04 \\ Arterial Stiffness and Wave Reflections in Relation to Plasma Advanced Glycation End Products in a Chinese Population

\author{
Qi-Fang Huang, Chang-Sheng Sheng, Ming Liu, \\ Fa-Hong Li, Yan Li, Ji-Guang Wang \\ The Shanghai Institute of Hypertension
}

Background: Accumulation of advanced glycation end products (AGEs) in the human body might engender arterial stiffening. We investigated the relationship of plasma AGEs concentration with arterial stiffness and wave reflections in a Chinese population.

Methods: The study subjects were recruited from a newly established residential area in the suburb of Shanghai in 2009. Using the SphygmoCor system, we measured carotidfemoral pulse wave velocity (cfPWV) and central (cAI) and peripheral augmentation indices (pAI). Plasma AGEs concentration was measured by the ELISA method and logarithmically transformed for statistical analysis.

Results: The 1051 study participants (mean age $55.1 \pm 13.1$ years) included 663 (63.1\%) women, 390 (37.1\%) hypertensive patients and $90(8.6 \%)$ diabetic or prediabetic subjects. Plasma AGEs concentration was higher in men than women (5.62 vs $5.07 \mu \mathrm{g} / \mathrm{mL}, \mathrm{P}=0.02)$ and with older age $(\mathrm{r}=$ 0.13 in both sexes, $\mathrm{P} \leq 0.01)$ and higher serum total-to-high density lipoprotein cholesterol ratio $(r=0.20$ in men and $r=$ 0.15 in women, $\mathrm{P}<0.0001)$. In multiple regression analyses, plasma AGEs concentration was significantly associated with cAI and pAI (1.9\% and 4.0\% increase per 10-time increase in plasma AGEs concentration, respectively, $\mathrm{P} \leq 0.02$ ) but not with cfPWV $(\mathrm{P}=0.62)$. However, there was significant $(\mathrm{P}=$ 0.001 ) interaction between plasma AGEs concentration and age in relation to cfPWV. Only in subjects of 70 years or older, cfPWV increased with higher levels of plasma AGEs concentration (bottom vs. top quintile distributions $8.10 \mathrm{vs.} 8.90 \mathrm{~m} / \mathrm{s}$, $\mathrm{P}=0.02$ ).

Conclusion: AGEs accumulate with aging and high cholesterol and are associated with arterial wave reflections and in an age-dependent manner also with arterial stiffness.

\section{M-05 \\ Lower Leg Muscle Mass is Associated with Augmentation Index but not with Brachial Blood Pressure in an Elderly Population}

\author{
Seung won Lee, Won Joon Lee, Wungrak Choi, \\ YooSik Youm, Yeong-Ran Park, Sang Hui Chu, \\ Hyeon Chang $\mathrm{Ki}$ \\ Department of Public Health, Yonsei University College \\ of Medicine, Seoul, Korea; Department of Preventive \\ Medicine, Yonsei University College of Medicine, Seoul, \\ Korea; Severance Hospital, Yonsei University Health \\ System, Seoul, Korea; Department of Sociology, Yonsei \\ University College of Social Sciences, Seoul, Korea; \\ Division of Silver Industry, Kangnam University, Yongin, \\ Korea; Department of Clinical Nursing Science, Yonsei \\ University College of Nursing, Seoul, Korea
}

Background: Augmentation index (AI), a measure of systemic arterial stiffness, was associated with coronary artery disease and cardiovascular risk. Previous crosssectional studies have shown an inverse relationship between muscle mass and arterial stiffness. The aim of this study was to investigate the association between lower leg muscle mass and the $\mathrm{AI}$ and brachial pressure in an elderly Korean population.

Methods: This study used data from Korean Social Life, Health and Aging Project (KSHAP) in 2011. A total of 814 people agreed to participate in the KSHAP, and 533 participants completed both questionnaire survey and health examinations. Leg muscle mass was measured with bioelectrical impedance analysis method by Inbody370 (Biospace, Seoul, Korea). AI was measured by HEM-9000AI (Omron Healthcare, Kyoto, Japan) and adjusted to heart rate of $75 \mathrm{bpm}$. Brachial pressure and anthropometric data were obtained from the participants. After excluding people missing AI measurements ( $\mathrm{n}=91)$, leg muscle mass $(\mathrm{n}=15)$, or laboratory tests $(\mathrm{n}=17)$, 180 men and 247 women aged 52-95 years were included in this cross-sectional study. The association between leg muscle mass and AI was investigated by multiple linear regression analysis.

Results : Mean (standard deviation) age was 71.7 (6.9) in men and 70.9 (7.2) in women. In male participants, leg muscle mass was inversely associated with AI when adjusted for age $(\beta=-2.641, p=0.001)$, and even after additional adjustment for body mass index, systolic and diastolic blood pressure, total cholesterol, HDL cholesterol, fasting glucose and insulin, smoking and alcohol consumption $(\beta=-1.632, p=0.035)$. In women, leg muscle mass was associated with AI when adjusted for age $(\beta=-1.540, p=0.033)$ but the association disappeared when fully adjusted $(\beta=-1.017, p=0.198)$. Leg muscle mass was not associated with brachial blood pressure in both men and women.

Conclusion: In an elderly Korean population, lower leg muscle mass was independently associated with arterial stiffness but not with brachial blood pressure. 


\section{M-06 \\ Risk of the Metabolic Syndrome in Normal BMI Korean in Prospective Cohort Study}

\author{
Juneyoung Yoon ${ }^{1}$, Juyong Lee ${ }^{2}$, Nam H. Cho ${ }^{3}$ \\ ${ }^{1}$ Department of Internal Medicine, Kurosungsim \\ Hospital, South Korea; ${ }^{2}$ St Elizabeths Medical Center of \\ Boston, USA; ${ }^{3}$ Department of Preventive Medicine, Ajou \\ University School of Medicine, Suwon, Korea
}

Background: The risk factors of the metabolic syndrome is known as obesity and lack of exercise. However, the metabolic syndrome in non-obese are common in Korea, where the prevalence of obesity is less than western. Insulin resistance and chronic inflammation is thought the reason of metabolic syndrome in non-obese people, but pathogenesis is not clear. Therefore, It is important which is the most initial risk factors of metabolic syndrome in normal BMI of the Korean population for screening and prevention. For this reason, we examined the risk factor of the metabolic syndrome in healthy person with normal BMI in prospective cohort study, who are going to have the metabolic syndrome after eight year.

Methods: A total 10,038 participants were recruited from the Ansung-Ansan cohort study. All subjects underwent healthy examination at baseline and at each biennial followup. 414 metabolic syndromes were diagnosed in normal BMI and previous healthy 1680 people during the period of eight years. The Cox proportional hazards model was used to analyze the risk factors of metabolic syndrome at 8 years.

Results: Baseline characteristics of the metabolic syndrome at 8 years follow-ups are age 40-49 163 people, age 50-59 135, age 60-69 116, non-exercise 295, exercise once a week 27 , exercise $2-3$ /week 35 , exercise everyday 57 , non-smoker 274, ex-smoker 48, current smoker ( $<1$ pack/ day) 39, current smoker (>1 pack/day) 53, none drinker 228, ex drinker 22, alcohol < $60 \mathrm{Kcal} 79$, alcohol $>60 \mathrm{Kcal} 85$, blood pressure $\geq 130 / 85 \mathrm{mmHg} 89$, waist circumference $\geq 102 \mathrm{~cm}$ or 40 inches (male), $\geq 88 \mathrm{~cm}$ or 36 inches (female) $144, \mathrm{TG} \geq$ $1.7 \mathrm{mmol} / \mathrm{L}(150 \mathrm{mg} / \mathrm{dl}) 214$, and HDL-C $<40 \mathrm{mg} / \mathrm{dL}$ (male), $<50 \mathrm{mg} / \mathrm{dL}$ (female) 259. The mean BMI is 23.1. First quartile BMI is 22.2. Second quartile and third quartile are 23.4 and 24.2. Age, gender, exercise frequency, family history of hypertension, family history of diabetes, family history of dyslipidemia, alcoho, smoking were not statistically significant. However, fasting plasma glucose $\geq 6.1 \mathrm{mmol} / \mathrm{L}(110 \mathrm{mg} / \mathrm{dl})$ risk ratio 2.60 , blood pressure $\geq 130 / 85 \mathrm{mmHg} 2.42$, central obesity $2.47, \mathrm{TG} \geq 1.7 \mathrm{mmol} / \mathrm{L}$ (150 mg/dl) 1.83, HDL-C $<40$ $\mathrm{mg} / \mathrm{dL}$ (male), $<50 \mathrm{mg} / \mathrm{dL}$ (female) 1.68 were statistically significant $(\mathrm{p}<0.000)$

Conclusion: Normal BMI population of metabolic syndrome is, even who has one criteria, greatly increased risk of developing after 8 years. Elevated in blood glucose levels or elevated blood pressure, as well as central obesity, the risk greatly increased. In conclusion, active lifestyles intervension or treatment should be considered who has only one criteria of metabolic syndrome.

\section{M-07 \\ Difference of Central Hemodynamic Parameters According to the Gender in General Population}

Chee Hae Kim, Byung-Hee Oh, Hae-Young Lee

Department of Internal Medicine, Seoul National

University College of Medicine

Background: Central as well as peripheral pulse pressure rises in both men and women according to natural aging process. It is quite interesting to see that women tend to have higher systolic blood pressure after menopause compared to the same age men, which somewhat explains the higher prevalence of cardiovascular complications in postmenopausal female than men. But the mechanism is not fully understood.

Methods: We used data from Korean Arterial Aging Study (KAAS) registry. KAAS registry enrolled 1,750 healthy subjects aged 17 to 80 years, in 22 university hospitals in Korea. Subjects are free of any medication for hypertension, diabetes and dyslipidemia.

\section{Results:}

1) Mean age is around 47 years old and BMIs are around 24.5 in males and 23 in females.

2) Central and peripheral systolic blood pressure was higher in men in average (central: $116.8 \pm 16.4 \mathrm{mmHg}$ versus $111.5 \pm 18.3 \mathrm{mmHg}, \mathrm{P}<0.001$, brachial: $128.5 \pm 16.7 \mathrm{mmHg}$ versus $120.1 \pm 18.3, \mathrm{P}<0.001$ ). Augmentation index (AIx) and aortic pulse wave velocity (aPWV) increase linearly with age. However, the slope of AIx might be stiffer in younger ages, whereas in case of aPWV, the slope is more stiff in older ages.

3) By multivariate analysis, AIx was independently correlated with age, diastolic blood pressure (BP), pulse pressure, and aPWV with systolic BP and age in men. For women, central AIx were independently correlated with age, diastolic BP and cholesterol level and aPWV with systolic BP, heart rate, fasting sugar, mean BP.

4) But when we combined the gender and the aging effects by dividing the age group into quartiles, there are sharp contrasts between man and women and between peripheral and central blood pressure. In younger ages, both central and peripheral pulse pressures were lower in females than in males. But around 45 years old, the pulse pressures of the women sharply rise, therefore in over fifties, women have higher pulse pressures compared with the men.

5) Same tendency of rapid increase in age associated vascular stiffness in female population was observed in central pulse wave velocity.

Conclusion: Women with smaller height, especially after menopause, have faster arrival time of reflected waves, higher augmentation index, and consequentially higher systolic blood pressure. This might be a fundamental mechanism for explaining the abrupt increase of the cardiovascular complications in postmenopausal women. 


\section{M-08 \\ Blood Hematocrit Level and Carotid Artery Intima Media Thickness in Men}

Sae Young Jae ${ }^{1}$, Kevin S. Heffernan ${ }^{2}$, Yoon-Ho Choi ${ }^{3}$

${ }^{1}$ Department of Sport Science, University of Seoul, Seoul, South Korea; ${ }^{2}$ Department of Exercise Science, Syracuse University, Syracuse, NY, USA; ${ }^{3}$ Center for Health Promotion, Samsung Medical Center, Sungkyunkwan University School of Medicine, Seoul, South Korea

Background: Increased hematocrit (Hct), a manifestation of increased in whole blood viscosity, is associated with the risk of all and cardiovascular disease mortality. Although several potential mechanisms have been put forth to explain the cardiovascular risks of high Hct, little is known on the association between increased Hct and carotid artery intima media thickness (CIMT), an index of subclinical vascular damage and advancing atherosclerosis. We investigated the hypothesis that higher levels of Hct are inversely associated with CIMT in 4684 (age $49 \pm 7$ yrs) healthy men.

Methods: We measured the common carotid artery intima media thickness using an ultrasound machine and carotid atherosclerosis was defined by carotid intima media thickness $>0.8 \mathrm{~mm}$. Fasting hematocrit levels were determined using a quantitative automated hematology analyzer.

Results: Hematocrit was independently associated with CIMT ( $\beta=0.12, p<0.01$ ) in multivariable regression models. Men in the highest quartile of Hct had significantly higher CIMT compared to men in the lowest quartile of Hct $0.59 \pm 0.13$ vs. $0.55 \pm 0.11 \mathrm{~mm}, \mathrm{p}<0.05$ ). The prevalence of carotid atherosclerosis was inversely associated with Hct across quartiles (Q1 (<43.5\%) 2.0\%, Q2 (43.5 45.5\%) 3.6\%, Q3 (45.6 47.3\%) $3.3 \%$, and Q4 (> 47.3\%) 5.0\%; p < 0.01 for trend). The odds ratios (OR) of the prevalence of carotid atherosclerosis in men with the highest quartile of Hct versus men with the lowest quartile of Hct was 2.65 (95\% CI, 1.57-4.47, p < 0.001) after adjustment for age, BMI, waist girth, SBP, TC, HDL-C, WBC, glucose, fibrinogen, HR and smoking. Each increment in Hct (1\%) was associated with a $10 \%$ (OR 1.10, 95\% CI 1.04-1.17, $\mathrm{p}=0.02$ ) increase in the prevalence of carotid atherosclerosis after adjustment for risk factors.

Conclusion: These results suggest that high Hct is associated with common carotid intima media thickening independent of established risk factors, which could contribute to high incidence of cardiovascular diseases in men with increased Hct level.

\section{M-09}

\section{A New Device, Enclosed Zone Flow-Mediated Vasodilation (ezFMD), is Useful for Assessment of Endothelial Function}

Masato Kajikawa ${ }^{1}$, Yukihito Higashi' ${ }^{2}$,Naomi Idei ${ }^{1}$, Shinsuke Mikami ${ }^{1}$, Tatsuya Maruhashi, Yumiko Iwamoto ${ }^{1}$, Takeshi Matsumoto ${ }^{7}$, Kensuke Noma², Toshio Tsuji ${ }^{3}$, Haruka Morimoto ${ }^{4}$, Teiji Ukawa ${ }^{4}$, Yasuki Kihara ${ }^{1}$

${ }^{1}$ Department of Cardiovascular Medicine, ${ }^{2}$ Department of Cardiovascular Regeneration and Medicine, Research Institute for Radiation Biology and Medicine, Hiroshima University, ${ }^{3}$ Department of Artificial Complex Systems Engineering, Hiroshima University, ${ }^{4} \mathrm{NIHON}$ KOHDEN Co.

Background: It is clinically important to estimate the degree of endothelial dysfunction. Recently, we developed a new noninvasive and simple method for measurement of vascular response to reactive hyperemia in the brachial artery, named enclosed zone flow-mediated vasodilation (ezFMD). The purpose of this study was to determine the validity of ezFMD for assessment of endothelial function.

Methods and Results: We measured ezFMD by a new device using an oscillometric method and conventional FMD using ultrasonography in 306 subjects, including patients with hypertension, dyslipidemia, and diabetes mellitus (218 men and 88 women, $30 \pm 16 \mathrm{yr}$ ). Univariate regression analysis revealed that ezFMD significantly correlated with age $(\mathrm{r}=$ $-0.42, \mathrm{P}<0.0001)$, body mass index $(\mathrm{r}=-0.13, \mathrm{P}=0.028)$, systolic blood pressure $(r=-0.15, P=0.009)$, diastolic blood pressure $(r=-0.14, P=0.011)$, fasting glucose level $(r=-0.27$, $\mathrm{P}=0.006)$, smoking $(\mathrm{r}=-0.21, \mathrm{P}=0.007)$ and baseline pulse wave amplitude $(\mathrm{r}=-0.51, \mathrm{P}<0.0001)$. ezFMD significantly correlated with FMD measured by ultrasonography $(\mathrm{r}=$ $0.34, \mathrm{P}<0.0001)$. Multiple regression analysis revealed that age $(\mathrm{P}=0.002)$, body mass index $(\mathrm{P}=0.013)$, systolic blood pressure $(\mathrm{P}=0.009)$, smoking $(\mathrm{P}=0.004)$ and baseline pulse wave amplitude $(\mathrm{P}<0.001)$ were independent predictors of ezFMD.

Conclusions: These findings suggest that measurement of ezFMD, a novel noninvasive and simple method, may be useful for determination of vascular diameter response to reactive hyperemia. Since ezFMD is automatically measured by a device with an oscillometric method, measurement of ezFMD is more simple and less bias than is measurement of conventional FMD. 


\section{M-10 \\ Impact of Diabetes Duration on the Severity and Extent of Coronary Atheroma Burden and Long-Term Clinical Outcome: Analysis by 128-Channel Coronary CT Angiography in Asymptomatic Diabetic Patients}

Jin Jin Kim, Sungmin Lim, Mineok Chang, Ik Jun Choi, Eun ho Choo, Jae Gyung Kim, Donggyu Moon, Byung-Hee Hwang, Pum Joon Kim, Kiyuk Chang, Wook Sung Chung, Ki-Bae Seung

Cardiovascular Center and Cardiology Division, Seoul St. Mary's Hospital, Seoul, Korea

Background: Coronary CT angiography (CCTA) is a highly accurate tool for detection of coronary artery disease. While diabetes may affect the progression of atherosclerosis, the association between diabetes duration, the severity and extent of coronary atheroma burden, and long-term clinical outcome has not been investigated in asymptomatic diabetic patients.

Methods: From 2005 to 2011, 2695 asymptomatic diabetic patients were enrolled. Of those, 924 patients underwent 128-channel CCTA. Patients were divided into three groups by diabetes duration: $<5$ years, $5-10$ years, and $>10$ years. We compared the severity and extent of coronary atheroma burden and major adverse cardiac and cerebrovascular events (MACCE) including cardiac death, nonfatal myocardial infarction (MI), and stroke during 5-year followup according between groups. The severity and extent of coronary artery disease burden was measured with multiple coronary CT angiographic scores, which are coronary artery calcium score (CACS), atheroma burden obstructive score (ABOS), segment involvement score (SIS), and segment stenosis score (SSS). Cox proportional hazards models were used to estimate the predictors of MACCE.

Results: Patients with longer duration of diabetes showed a greater level of CACS ( $p<0.001)$, ABOS ( $p<0.001)$, SIS ( $p<0.001)$, and SSS ( $p<0.001)$ in CCTA, and also showed a higher risk of MACCE $(p=0.001)$. However, in multivariable Cox regression analysis, ABOS (hazard ratio[HR] 1.248, 95\% confidence interval[CI] 1.077-1.445, p = 0.003), SIS (HR 1.136, 95\% CI 1.027-1.156, $\mathrm{p}=0.004$ ) and SSS (HR 0.004, 95\% CI 1.027-1.156, $\mathrm{p}=0.004$ ) except CACS (HR 1.000, 95\% CI $1.000-1.001, p=0.189$ ) in CCTA were significantly associated with the risk of MACCE.

Conclusions: In asymptomatic diabetic patients, longer diabetes duration is proportionately associated with the severity of coronary atheroma burden on CCTA and the risk of MACCE. Furthermore, severe coronary atheroma burden score is closely related with elevated risk of MACCE. Coronary artery atheroma burden score were more closely related with MACCE than CACS.

\section{M-11 \\ Comparison of Accuracy of Three Methods for Measuring Central Systolic Blood Pressure with A Brachial Pressure Waveform}

Hao-Min Cheng 1,2,3, Yuan-Ta Shih ${ }^{5}$, Shih-Hsien Sung 1,3,4, Chen-Huan Chen ${ }^{1,2,3,4}$

${ }^{1}$ Department of Medicine, and ${ }^{2}$ Department of Medical Research and Education, Taipei Veterans General Hospital, ${ }^{3}$ Department of Medicine, ${ }^{4}$ Department of Public Health, Faculty of Medicine, National Yang-Ming University, Taipei, Taiwan, R.O.C.; ${ }^{5}$ Molecular imaging center, National Taiwan University

Background: Central systolic blood pressure (SBP-C) can be estimated from a peripheral blood pressure waveform using generalized transfer function (GTF), pulse waveform analysis (PWA), or N-points moving average (NPMA) methods. The aim of this study was to compare the measurement accuracy of the three methods.

Methods: Simultaneous invasive high-fidelity right brachial, central aortic pressure waveforms and non-invasively left brachial systolic and diastolic pressures from a validated oscillometric blood pressure monitor were recorded in 40 subjects. Measured and estimated values of SBP-C were obtained from the invasive pressure waveforms. Subsequently, the invasive brachial pressure waveforms were recalibrated with the non-invasive systolic and diastolic pressures to introduce a calibration error.

Results: The non-invasive brachial systolic blood pressure underestimated the invasive brachial pressure by $-2.3 \pm 5.8 \mathrm{mmHg}$. The mean differences between the estimated and measured SBP-C by using GTF, PWA and NPMA were -1.2 $\pm 2.6 \mathrm{mmHg}, 0 \pm 3.1 \mathrm{mmHg}, 0 \pm 3.5 \mathrm{mmHg}$, respectively, and $-2.7 \pm 6.1 \mathrm{mmHg},-1.3 \pm 5.3 \mathrm{mmHg}$ and $-1.6 \pm 7.0 \mathrm{mmHg}$ after the recalibration, respectively. SBP-C estimated by GTF was significantly lower than that by PWA and NPMA, before and after the calibration (all $P<0.03$ ). In contrast, the variance of SBP-C difference by NPMA was significantly greater than that by GTF before the calibration, and was significantly greater than that by PWA after the calibration (all $\mathrm{P}<0.05$ ). The error transfer from the calibration was $117 \%$ by GTF, $56 \%$ by PWA, and $69 \%$ by NPMA, respectively.

Conclusions: the GTF method underestimates the invasively measured SBP-C, whereas the NPMA method produces greater variance than the GTF and PWA methods. With calibration to the noninvasive brachial blood pressures, the GTF method may amplify whereas PWA and NPMA may reduce the calibration error. The results have important implications for the application of the cuff-based noninvasive techniques for estimating SBP-C. 


\section{M-12}

\section{The Effect of Mitral Regurgitation and Cardiac} Fibrosis on Ventricular Remodeling and Exercise Capacity in a Murine Model of Left Ventricular Pressure Overload

Kyung-Hee Kim, Yong-Jin Kim, Seung-Pyo Lee, Hyung-Kwan Kim, Dae-won Sohn, Byung-Hee Oh

Seoul National University Hospital, Seoul, Korea

Background: Degenerative mitral regurgitation (MR) is often accompanied by hypertension (pressure overload) with aging. This study determined the contribution of volume overload and cardiac fibrosis on left ventricular (LV) remodeling and exercise capacity in rats with pressure overloaded rats.

Method: Pressure overloaded and left ventricular hypertrophy (LVH) were created by suprarenal aortic constriction (SAC) in SD rats. Serial echocardiographic studies and exercise tests were performed at 2-week intervals and invasive hemodynamic examination by a pressure-volume catheter system was done at 14 weeks after SAC. The rats were divided into 4 groups [SAC, SAC+MR = SAC followed by MR operation, MR = laparotomy followed by MR operation, Control]. MR was created by introducing a needle through LV apex and making a hole on anterior mitral leaflet. SAC was done at 2 weeks before MR formation in SAC+MR group.

Results: LV wall thickness increased in SAC group compared to control group whereas LV ejection fraction (EF) and E/E' did not change. LV end diastolic pressure and the EDPVR slope were greater in SAC group than in control group. LV dilatation and exercise intolerance were developed first in SAC+MR group. However, MR group showed a catch-up of remodeling and exercise intolerance at 10 weeks after MR formation (LV ESD at 10 weeks after MR formation, $4.33 \pm 0.26$ vs. $6.50 \pm 0.40$ vs. $6.59 \pm 1.38 \mathrm{~mm}$ for control vs. MR vs. SAC+MR, $\mathrm{P}<0.05$; LV EDD, $7.68 \pm 0.15$ vs. $10.48 \pm 0.46$ vs. $10.20 \pm 1.26$ $\mathrm{mm}, \mathrm{P}<0.05$ exercise duration, $765.3 \pm 130.1$ vs. $487.8 \pm 49.0$ vs. $434.0 \pm 80.3$ seconds, $\mathrm{P}<0.05$ no statistical differences between SAC+MR and MR). Myocardial fibrosis was greatest in SAC and greater in SAC+MR compared with MR and control.

Conclusion: Induction of volume overload before cardiac fibrosis in pressure overloaded rats affected LV compliance and improved exercise capacity over time in rats. Cardiac fibrosis was correlated with EDPVR and was higher in pressure overloaded rats compared with MR.

\section{M-13 \\ Effects of Aging on Ascending Aortic Flow Waveform}

Michael O'Rourke 1,2, Audrey Adji1,3, Nadjia Kachenoura4, Emilie Bollache ${ }^{4,5}$, Elie Mousseaux ${ }^{4,6}$, Alberto Avolio $^{3}$

${ }^{1}$ St Vincent's Clinic, Sydney, Australia; ${ }^{2}$ University of New South Wales/ VCCRI, Sydney, Australia; ${ }^{3}$ Australian School of Advanced Medicine, Macquarie University, Sydney, Australia; ${ }^{4}$ Laboratoire d'Imagerie Fonctionnelle, Paris, France; ${ }^{5}$ Institut Jean le Rondd'Alembert, Paris, France; ${ }^{6}$ Cardiovascular Imaging Department, Hôpital Européen Georges Pompidou, AP-HP, Paris, France

Background: Limited invasive (electromagnetic) ascending aortic (AA) flow wave contour indicates impaired left ventricular (LV) function in the elderly. However, conventional non-invasive (Doppler) recordings have not been confirmed to show similar aging change. This study aims to illustrate changes in AA flow and carotid pressure waves in an apparently normal cohort, using Cardiac Magnetic Resonance Imaging (CMRI).

Methods: Forty apparently normal subjects (21-70 years, 21 males) underwent CMRI of the thoracic aorta using a 1.5 T system (Signa, GEMS, Waukesha, USA). DR $1 \frac{1}{3}$ was determined as ratio of difference in flow velocity between peak and one-third of deceleration phaseto peak flow velocity (from Miyashita et al, 1994). Comparison was made between young $(<50$ years, $\mathrm{n}=27$ ) and older cohort ( $>50$ years, $\mathrm{n}=13$ ).

Results: Peak AA flow velocity decreased substantially $(\mathrm{p}<0.0001)$ from young $(67.6 \pm 18.3 \mathrm{~cm} / \mathrm{s})$ to older subjects $(43.9 \mathrm{~cm} / \mathrm{s})$. This was largely attributable to aortic dilation. However, late systolic flow was relatively lower in the older than in the younger subjects, with flow concavity in older persons corresponding to greater augmentation of

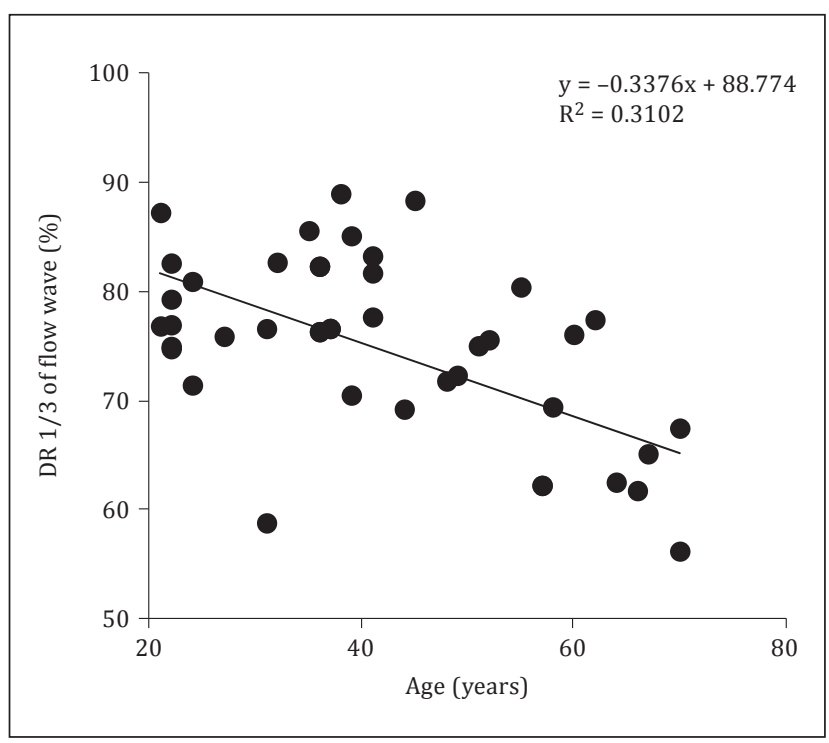

Fig. 1. (for Abstract M-13). 
the pressure waveform, represented as $\mathrm{DR}^{1} \frac{1}{3}$ significant ( $\mathrm{p}=$ 0.003 ) decline from $78 \%(\leq 50 y$ ) to $67 \%$ ( $>50 y$ ). These changes are explicable on the basis of LV impairment from early and increased wave reflection during systole.

Conclusions: AA flow waves recorded by CMRI showed aging changes which are not apparent in conventional Doppler flow patterns. Our finding warrants further use of AA CMRI flow and pressure waveforms to characterise aging changes and ill-effects of stiffening of the central arteries. Findings support the theoretic predictions of Westerhof and O'Rourke (J Hypertens 1995;13:943-952) and the data interpretations of Miyashita et al. (Heart Vessels 1994;9:30-39).

\section{M-14 \\ The Mechanical Properties of Common Carotid Artery Accessed by Speckle Tracking were Associated with Ventricular Dysfunction and Incident Heart Failure}

\author{
Jo-Nan Liao ${ }^{1,3}$, Shih-Hsien Sung 1,3,4*, Wen-Chung $\mathrm{Yu}^{1,3}$, \\ Hao-Min Cheng 2,3, Chen-Huan Chen ${ }^{2,3,4}$ \\ ${ }^{1}$ Division of Cardiology, Department of Medicine, and \\ ${ }^{2}$ Department of Medical Research and Education, Taipei \\ Veterans General Hospital; ${ }^{3}$ Cardiovascular Research \\ Center, and ${ }^{4}$ Institute of Public Health and Community \\ Medicine Research Center, National Yang-Ming \\ University, Taipei, Taiwan
}

Background: Arterial stiffness accessed generally by pulse wave velocity or locally at common carotid artery (CCA) has been introduced as a risk of cardiovascular disease. The present study investigated the correlations of mechanical properties of CCA using speckle-tracking technique with cardiac performance and incidence of heart failure.

Methods: A total of 114 subjects (aged 63.5 \pm 17.5 years) presented with dyspnea to the outpatient clinics were enrolled. Comprehensive echocardiography, carotid artery sonogram, and measures of carotid-femoral pulse wave velocity (cf-PWV), carotid augmentation index (cAI), incremental modulus of elasticity (Einc) of CCA, carotid distensibility (CD), and $\beta$ stiffness index were performed. Carotid circumferential strain (CS) and strain rate (CSr) were also calculated. The mean intra-observer and inter-observer agreements were $99.93 \pm 0.08 \%$ and $99.91 \pm 0.1 \%$ for carotid CS, and $99.92 \pm 0.07 \%, 99.91 \pm 0.09 \%$ for carotid CSr.

Results: Carotid CS was significantly associated with left ventricular ejection fraction (LVEF) $(r=0.287)$, ratio of early to late transmitral flow velocity $(\mathrm{E} / \mathrm{A}, \mathrm{r}=0.224)$, and ratio of transmitral flow velocity to septal mitral annulus tissue velocity at early diastole ( $\left.E / E^{\prime}, r=0.285\right)$. However, carotid CSr significantly correlated with $\mathrm{E} / \mathrm{A}(\mathrm{r}=0.331)$ and $\mathrm{E} / \mathrm{E}^{\prime}(\mathrm{r}=$ -0.222 ) but not LVEF. In the study population, 51 subjects had either left ventricular systolic $(n=25)$ or diastolic dysfunction $(\mathrm{n}=26)$. Carotid CS and CSr, as well as cf-PWV, carotid Einc, $\mathrm{CD}, \beta$ stiffness index, but not cAI, were independently related to the presence of abnormal ventricular function, after accounting for age, sex, and brachial systolic blood pressure. When E/E' was further adjusting for, only carotid CS was the independent predictor of impaired ventricular function. In addition, low carotid CS was associated with high incidence of acute heart failure during a follow period up to 1 year (log rank $\mathrm{p}=0.006$ ).

Conclusion: Mechanical properties of CCA accessed by speckle tracking were associated with cardiac performance, presence of either systolic or diastolic ventricular dysfunction, and incidence of acute heart failure.

\section{M-15 \\ Arterial Stiffness/Central Hemodynamics, Renal Function, and the Development of Hypertension \\ Hirofumi Tomiyama, Chisa Matsumoto, Mari Odaira, Jiko Yamada, Masanobu Yoshida, Kazuki Shiina, Akira Yamashina \\ Department of Cardiology Tokyo Medical University, Japan}

Objectives: This prospective study was conducted in 1229 middle-aged Japanese men with preserved renal function without hypertension, to examine whether the arterial stiffness/central hemodynamic parameters measured at baseline, and the renal function measured at baseline, might be independently associated with the future development (at the end of the 3 years' study period) of hypertension without interaction; we also attempted to clarify that any association of the initial arterial stiffness/central hemodynamic parameters with the future development, 3 years later, of hypertension would be independent of the renal function decline at the end of the 3-year period.

Methods: The estimated glomerular filtration rate calculated based on the serum creatinine or serum cystatin C (eGFRcr, eGFRcys), brachial-ankle pulse wave velocity (baPWV), and radial augmentation index ( $\mathrm{rAI}$ ) were measured at the baseline and at the end of the 3-year study period.

Results: Hypertension was detected at study completion in 127 men. According to the results of multiple logistic regression analysis, eGFRcys, but not the eGFRcr, and either the baPWV or rAI at the initial examination showed a significant independent adjusted odds ratio for the presence of hypertension at the end of the 3-year study period, without any interaction \{adjusted adds ratio of baPWV (per standard deviation increase) = 2.054 (1.551-2.720), $\mathrm{p}<0.001$; that of eGFRcys (per standard deviation decrease $)=1.643(1.186-2.276), p=0.003\}$. The results of the multivariate linear regression analysis showed that neither the initial baPWV nor the initial rAI was related to the renal function at the end of the 3-year study period.

Conclusions: The initial arterial stiffness/central hemodynamic parameters and initial eGFR may be independently associated with the future development of hypertension, without any interaction. Furthermore, the association of the initial arterial stiffness/central hemodynamic parameters 
with the future development of hypertension may be direct, and independent of the renal functional decline detected at the corresponding time-point.

\section{M-16 \\ Different Clinical and Angiographic Characteristics According to Vessel Size in Patients Undergoing Acetylcholine Provocation Test \\ Se Yeon Choi, Seung-Woon Rha, Byoung Geol Choi, Cheol Ung Choi, Chang Gyu Park, Hong Seog Seo, Dong Joo Oh \\ Cardiovascular Center, Korea University Guro Hospital, Seoul, Korea}

Background: There are limited data regarding angiographic and clinical characteristics according to different reference vessel size during intracoronary acetylcholine (Ach) provocation test to evaluate coronary artery spasm (CAS) in patients (pts) with suspected vasospastic angina.

Methods: A total 2021 consecutive pts, who underwent intracoronary Ach provocation test were enrolled. Provocation test was performed by incremental dosages $(20,50,100 \mathrm{ug})$ of Ach until get significant response ( $>70 \%$ narrowing). The study population were divided into small vessel group (< $2.5 \mathrm{~mm}$ ) and large vessel group ( $\geqq 2.5 \mathrm{~mm}$ ) and angiographic and clinical parameters during Ach provocation test were compared between the two groups.

Results: Baseline characteristics were similar between the two groups except that large vessel group had more male gender ( $56 \%$ vs. $47.4, \mathrm{p}<0.001)$, peripheral vascular disease ( $4.1 \%$ vs. $2.3 \%, \mathrm{p}=0.025)$, alcoholics (39.7\% vs. $30.5 \%, \mathrm{p}<$ 0.001 ) and less diabetics (8.5 vs. 13.9, p < 0.001). During Ach provocation test, large vessel group had a higher incidence of baseline spasm (> 30\%) and multi-vessel spasm, whereas small vessel group had higher incidence myocardial bridge and diffuse spasm (table).
Conclusion: According to the current study, in pts with vasospastic angina, large reference vessel was associated with increase basal spasm and multivessel spasm; whereas small reference vessel was associated with diffuse spasm.

M-17

A Randomized Controlled Study to Effects
of Bisoprolol and Atenolol on Sympathetic
Nervous Activity and Central Aortic Pressure
in Patients with Essential Hypertension

Weijun Zhou ${ }^{1,2,3}$, Renying Wang ${ }^{1,2}$, Yan $L i^{1,2}$, Dongrui Chen 1,2, Erzhen Chen ${ }^{4}$, Dingliang Zhu ${ }^{1,2}$, Pingjin $\mathrm{Gao}^{1,2,3}$

${ }^{1}$ State Key Laboratory of Medical Genomics, Shanghai Key Laboratory of Hypertension and Department of Hypertension, Ruijin Hospital, Shanghai Jiao Tong University School of Medicine, Shanghai 200025, China; ${ }^{2}$ Shanghai Institute of Hypertension, Shanghai 200025, China; ${ }^{3}$ Laboratory of Vascular Biology, Institute of Health Sciences, Shanghai Institutes for Biological Sciences, Chinese Academy of Sciences, Shanghai 200025, China; ${ }^{4}$ Department of Emergency, Ruijin Hospital, Shanghai Jiao Tong University School of Medicine, Shanghai 200025, China

Background: $\beta$-blockers (BBs) with different pharmacological properties may have heterogeneous effects on sympathetic nervous activity (SNA) and central aortic pressure (CAP), which are independent cardiovascular factors for hypertension. Hence, we analyzed the effects of bisoprolol and atenolol on SNA and CAP in hypertensive patients.

Methods: This was a prospective, randomized controlled study with 109 never-treated hypertensive subjects randomized to bisoprolol ( $5 \mathrm{mg}$ ) or atenolol (50 $\mathrm{mg}$ ) for $4 \sim 8$ weeks. To evaluate SNA, baroreflex sensitivity (BRS) and heart rate variability (HRV) were measured by power spectral analysis using a Finometer. CAP and related parameters were determined with the SphygmoCor apparatus using pulse wave analysis.

Table 1. Clinical and angiographic parameters of the study groups (for Abstract M-16)

\begin{tabular}{|c|c|c|c|}
\hline Variables, n (\%) & Small vessel $(n=1257)$ & Large vessel $(n=764)$ & P-value \\
\hline \multicolumn{4}{|l|}{ Clinical parameters } \\
\hline EKG change & $60(4.8)$ & $47(6.2)$ & 0.180 \\
\hline Chest pain & $819(65.2)$ & $479(62.7)$ & 0.263 \\
\hline \multicolumn{4}{|l|}{ Angiographic characteristics } \\
\hline Myocardial bridge & $364(29.0)$ & $140(18.3)$ & $<0.001$ \\
\hline Diffuse lesion (> 30mm) & $1079(85.8)$ & $617(80.8)$ & 0.003 \\
\hline Multi vessel spasm & $364(29.0)$ & $299(39.1)$ & $<0.001$ \\
\hline Baseline spasm (> 30\%) & $302(24.0)$ & $222(29.1)$ & 0.012 \\
\hline Reference Diameter-NTG & $2.04 \pm 0.32$ & $3.01 \pm 0.4$ & $<0.001$ \\
\hline
\end{tabular}


Results: Both drugs were similarly effective in reducing brachial BP. However, central systolic BP $(-14 \pm 10 \mathrm{~mm}$ Hg vs $-6 \pm 9 \mathrm{~mm} \mathrm{Hg} ; \mathrm{P}<0.001)$ and aortic pulse pressure $(-3 \pm 10$ $\mathrm{mm}$ Hg vs $+3 \pm 8 \mathrm{~mm} \mathrm{Hg}$; $<0.001$ ) decreased significantly with bisoprolol than with atenolol. The augmentation index at a heart rate (HR) of $75 \mathrm{bpm}$ (AIxatHR75) was significantly decreased $(29 \% \pm 11 \%$ to $25 \% \pm 12 \%$; $\mathrm{P}=0.026)$ only in the bisoprolol group. Furthermore, the change in BRS in the bisoprolol group $(3.99 \pm 4.19 \mathrm{~ms} / \mathrm{mmHg})$ was higher than that in the atenolol group $(2.66 \pm 3.78 \mathrm{~ms} / \mathrm{mmHg})$, although not statistically significant $(\mathrm{P}>0.05)$. BRS was stable when RHR was controlled (RHR $\leq 65 \mathrm{bpm}$ ), and the two treatments had similar effects on low frequency (LF)/high frequency (HF) ratio and on $\mathrm{HF}$.

Conclusion: BBs seem to have dissimilar effects on arterial distensibility and compliance in hypertensive subjects. Compared with atenolol, bisoprolol may have a better effect on CAP.

\section{M-18 \\ Widened Pulse Pressure in True Resistant Hypertension: An Observation from the Chinese Ambulatory and Home Blood Pressure Registry}

Yuan-Yuan Kang, Yan Li, Qi-Fang Huang, Qian Dong, Jie-Song, Ji-Guang Wang

Center for Epidemiological Studies and Clinical Trials, Shanghai Key Lab of Hypertension, Shanghai Institute of Hypertension, Ruijin Hospital, Shanghai Jiao Tong

University School of Medicine, Shanghai, China

Background: Resistant hypertension was associated with adverse cardiovascular outcome. The aim of this study was to investigate if patients with true resistant hypertension would have a stiff artery as measured by pulse pressure.

Methods: From August 2009 to December 2012, 2015 patients were registered in the Chinese Ambulatory and Home Blood Pressure Registry. True resistant hypertension was defined as uncontrolled blood pressure in both office ( $\geq$ $140 / 90 \mathrm{~mm} \mathrm{Hg}$, for diabetic patients, $\geq 130 / 80 \mathrm{~mm} \mathrm{Hg}$ ) and 24-hour ambulatory monitoring ( $\geq 130 / 80 \mathrm{~mm} \mathrm{Hg}$ ) settings despite optimal treatment with at least 3 antihypertensive drugs including a diuretic. The 24-hour pulse pressure was the difference between the 24-hour systolic and diastolic blood pressures. We performed the analysis of variance to compare the means of pulse pressure between groups while accounting for sex, age, body mass index, current smoking and drinking habit, mean blood pressure and the presence of diabetes.

Results: Among the 2015 registered subjects, 18 had missing office blood pressure measurements, leaving 1997 (53.5\% men, average age 56 years) subjects in the current analysis. Among all subjects, 1772 had hypertension, 1334 were treated with antihypertensive drugs, in whom 661 (49.6\%) had both office and 24-hour ambulatory blood pressure controlled, and $40(2.9 \%)$ had true resistant hypertension.
Compared to controlled hypertensive patients, patients with true resistant hypertension had greater body mass index (27.4 vs. 24.4, $\mathrm{P}<0.001)$ and included more smokers $(30.0 \%$ vs. 9.8\%, $\mathrm{P}<0.001)$. After multivariate adjustment, patients with true resistant hypertension had significantly higher 24-hour pulse pressure (54.9 vs. $46.6 \mathrm{~mm} \mathrm{Hg}, \mathrm{P}<0.001$ ) than patients with controlled hypertension. Logistic regression analysis showed that each $5 \mathrm{mmHg}$-increase in 24-hour pulse pressure was associated with $87 \%$ (95\%CI: $22 \%-186 \%, \mathrm{P}=$ 0.0039 ) higher odds of true resistant hypertension.

Conclusion: Patients with true resistant hypertension had widened pulse pressure than patients with controlled hypertension. Stiff arteries might render hypertension more difficult to control.

\section{M-19 \\ The Parasympathetic Activity Modulating Arterial Stiffness is Associated with Orthostatic Hypotension \\ Dai-Yin Lu, Shih-Hsien Sung, Wen-Chung Yu, Hao-Min Cheng, Shao-Yuan Chuang, Chen-Huan Chen \\ Taipei Veterans General Hospital}

Background: The baroreflex-mediated autonomic responses, which are essential to maintain blood pressure during postural changes, are controlled in part by both the sympathetic and parasympathetic nervous systems. We therefore investigated how autonomic system involves the presence of orthostatic hypotension $(\mathrm{OH})$.

Methods: A total of 427 subjects $(67.7 \pm 15$ years, $76.81 \%$ men) were enrolled in this study. Measurements of carotid systolic blood pressure (SBP) and pulse pressure (PP), carotid-femoral (cf-PWV) and brachial-ankle pulse wave velocities (ba-PWV), augmentation index (AI), carotid augmented pressure (CAP), and amplitude of the reflected pressure wave from a decomposed carotid pressure wave $(\mathrm{Pb})$ by carotid and femoral tonometry were obtained. Frequency domain measures of heart rate variability, including very low frequency (VLF), low frequency (LF), high frequency (HF), and the ration of low to high frequency measures (LHR) were calculated by five-minutes recording of heart rate at supine.

Results: The 50 patients with $\mathrm{OH}$ were more likely to be diabetic, had lower estimated glomerular filtration rate and more anti-hypertensives comparing to the other 377 subjects without $\mathrm{OH}$. However, the distributions of age, gender, hypertension, stroke, and coronary artery disease were similar. Furthermore, subjects with $\mathrm{OH}$ had higher baseline brachial and carotid SBP and PP, cf-PWV, ba-PWV, cAP, and Pb (all P < 0.05), butnotAI.Theheartratevariability, includingVLF, LF, and $\mathrm{HF}$, but not LHR was significantly lower in individuals with $\mathrm{OH}$. With adjustment of age, both VLF and HF (marker of parasympathetic modulation) were negatively related to cf-PWV (standardized coefficients, $\beta=-0.113$ and $-0.120, P=0.023$ and 0.016 , respectively. In multivariate analysis adjusting for age, DM and eGFR, only HF was significantly associated with 
the presence of OH (HR 0.643, 95\% CI 0.427-0.969), an association that went away after further correcting for cf-PWV.

Conclusion: The parasympathetic rather than the sympathetic activity was significantly associated with $\mathrm{OH}$ through its modulation of arterial stiffness.

\section{M-20 \\ Effects of Nifedipine and Valsartan versus High Dose Monotherapy on Central Hemodynamics in Patients with Inadequately Controlled Hypertension: FOCUS Study}

Jeong Bae Park ${ }^{7}$, Jong-won Ha ${ }^{2}$, Hae-Ok Jung ${ }^{3}$, MooYong Rhee 4 , FOCUS Investigators

${ }^{1}$ Division of Cardiology, Cheil General Hospital, Kwandong University College of Medicine, Seoul; ${ }^{2}$ Division of Cardiology, Severance Hospital, Yonsei University College of Medicine, Seoul; ${ }^{3}$ Division of Cardiology, The Catholic University of Korea, St. Mary's Hospital, Seoul; ${ }^{4}$ Division of Cardiology, Dongguk

University Hospital, Goyang-si, Gyeonggi-do, Korea

Objectives: This study investigated whether the low-dose combination of nifedipine GITS and valsartan is more effective in reducing central blood pressure (BP) than high-dose monotherapy with either agent in essential hypertension inadequately controlled by low-dose monotherapy.

Background: Measures of central BP and arterial stiffness hold prognostic information beyond conventional peripheral BP. Calcium channel or renin-angiotensin system blockers have shown better efficacy in improving central hemodynamics among antihypertensives, but there are currently no direct comparisons.

Methods: This is a prospective, open-label, randomized, active-controlled study, including 203 patients with uncontrolled hypertension. Patients were randomized to receive 30 $\mathrm{mg}$ nifedipine GITS plus $80 \mathrm{mg}$ valsartan (N30+V80), $60 \mathrm{mg}$ nifedipine GITS (N60), or 160 mg valsartan (V160) for 8 weeks. Central hemodynamics were measured by applanation.

Results: The primary efficacy variable, central systolic $\mathrm{BP}$, compared to baseline at week 8 was significantly reduced by $27.2 \pm 14.7 \mathrm{~mm} \mathrm{Hg}$ in the N30+V80 group and by $27.1 \pm 16.5$ $\mathrm{mm} \mathrm{Hg}$ with $\mathrm{N} 60$ group compared to $14.4 \pm 16.6 \mathrm{~mm} \mathrm{Hg}$ with V160 group. The decrease in augmentation index in the N60 group was statistically significant compared to V160 alone ( $\mathrm{P}=0.0464)$, without significant differences between combination therapy and either high-dose monotherapy. Pulse pressure amplification was significantly increased in the N30+V80 group, but not in N60 or V160 group. By multivariate regression analysis, changes in central systolic BP are determined by baseline pressure and drugs $(\mathrm{P}<0.001)$.

Conclusions: Low-dose combinations of nifedipine GITS plus valsartan or high-dose nifedipine were more effective in improving central hemodynamics than high-dose valsartan in patients with hypertension.

\section{M-21 \\ Central Blood Pressure, Pulse Wave Velocity and Carotid Artery Intima-Media Thickness During Strict or Mild Home Blood Pressure Control with Amlodipine- or Losartan- Based Regimen in Hypertensive Patients: Hypertension Control Based on Home Systolic Pressure (HOSP) Substudy \\ Yuko Ohta, Hideaki Takata, Yoshio Iwashima, Shinichiro Hyashi, Fumiki Yoshihara, Satoko Nakamura, Yuhei Kawano \\ Division of Hypertension and Nephrology, National Cerebral and Cardiovascular Center}

Objectives: To compare the effect of central blood pressure (BP), brachial-ankle pulse wave velocity (baPWV) and carotid artery intima-media thickness (IMT) during strict and mild control of morning home systolic BP (SBP) with amlodipine- and losartan-based regimen in hypertensive patients

Methods: Subjects included 80 hypertensive outpatients who participated in the HOSP pilot study. After a one-month drug-free period, patients were randomly assigned to either the strict control group (target morning home SBP $<130$ $\mathrm{mmHg}$ ) or the mild control group (130-139 $\mathrm{mmHg}$ ) and to either the amlodipine or losartan group. Additional antihypertensive drugs were added if target BP was not achieved with monotherapy. Central BP and baPWV were measured after 5 years in 32 patients and carotid IMT was measured after 5 years in 54 patients. PWV was measured using form $\mathrm{ABI} / \mathrm{PWV}$ (Omron-Colin), and central BP was measured using HEM-9000AI (Omron-Colin).

Results: Morning home SBP achieved target levels during 5 years in the strict control group as well as in the mild control group, while it was comparable between amlodipine and losartan groups. Central BP and changes in PWV were lower in the strict control group than the mild control group, however, the difference was not statistically significant at year 5 . Those measures were not different between amlodipine and losartan groups. In all patients, mean and max carotid IMT increased significantly. Changes in carotid IMT did not differ between strict and mild control groups. The increase in mean carotid IMT in the amlodipine group were smaller than those in the losartan group at year 1 , but were not different between the two groups at year 5 .

Conclusion: Central BP and PWV tended to decrease in strict control group compared to mild control after five years. Carotid IMT increased over time in hypertensive patients in spite of the strict control of home BP. Amlodipine may slow the progression of IMT compared to losartan, although the difference was not obvious after five years. 


\section{M-22 \\ CPAP Improves Visit-to-Visit Blood Pressure Variability in Patients with Severe Obstructive Sleep Apnea}

Kazuki Shïna, Hirofumi Tomiyama, Yoshifumi Takata, Masanobu Yoshida, Mari Odaira, Kota Kato,

Nishihata Yosuke, Tasuku Yamaguchi, Yasuhiro Usui, Akira Yamashina

Department of Cardiology, Tokyo Medical University, Tokyo, Japan

Background and Aim: Recent studies have shown that visit-to-visit blood pressure variability (VVV) is powerful risk factor for cardiovascular disease. Obstructive sleep apnea (OSA) was associated with high blood pressure variability hypertension (eg, mornig surge, masked hypertension, resistant hypertension) and cardiovascular risk. The aim of this study was to investigate the effect of continuous positive airway pressure (CPAP), standard therapy for severe OSA, on VVV in patients with obstructive sleep apnea.

Method: Thirty seven OSA patients (mean age $57 \pm 11$ ) assessed by Polysomnography (mean AHI 53 $\pm 22 / \mathrm{h}$ ) were enrolled. Blood pressure was measured 10 times at sleep outpatient clinic every 1 month before and after CPAP. VVV were expressed as the standard deviation (SD) of systolic blood pressure and coefficient of variation (CV).

Result: CPAP significantly reduced systolic blood pressure and maximum systolic blood pressure $(137.3 \pm 13.9 \rightarrow$ $128.8 \pm 9.0 \mathrm{mmHg}, \mathrm{P}<0.001,155.4 \pm 21.5 \rightarrow 134.7 \pm 9.9 \mathrm{mmHg}$, $\mathrm{P}<0.001$, respectively). VVV parameters, SD and CV, were significantly decreased $(14.1 \pm 7.7 \rightarrow 4.8 \pm 2.8 \mathrm{mmHg}, \mathrm{P}<0.001$, $10.2 \pm 5.2 \rightarrow 3.8 \pm 2.2 \%, \mathrm{P}<0.001$, respectively).

Conclusion: CPAP significantly reduced systolic blood pressure, maximum systolic blood pressure and VVV in patients with OSA. Next logical step is to clarify whether this improvemen of VVV by CPAP therapy is associated with the improvement of the prognosis in subjects with OSA.

\section{M-23 \\ Statin Improves Flow-Mediated Dilation in Patients with Chronic Kidney Diseases}

Tsuneo Takenaka, Yoichi Ohno, Hiromichi Suzuki

Department of Nephrology, Community Health Science Center, Saitama Medical University, Japan

Background and Aim: Many drugs are required to manage patients with chronic kidney diseases (CKD). Thus, drug compliance is relatively poor in this population. The impact of combination drug on compliance was examined. Subjects were 15 (7 males) CKD patients with hypertension and dyslipidemia, who were visiting our outpatient office and taking amlodipine $5 \mathrm{mg}$ and atorvastatin $10 \mathrm{mg}$ daily. The influences of exchanging to a combination drug (Caduet) containing equivalent doses of amlodipine and atorvastatin were observed.

Method: Central blood pressure was estimated with radial artery tonometry, and flow-mediated dilation (FMD) was measured using echography on brachial artery.

Result: Patient backgrounds were as follows: mean age $63 \pm 3$ y/o, serum creatinine $1.37 \pm 0.11 \mathrm{mg} / \mathrm{dl}$, protein excretion $0.49 \pm 0.06 \mathrm{~g} / \mathrm{gCr}$, blood pressure $134 \pm 2 / 74 \pm 1 \mathrm{mmHg}$ and pulse rate $74 \pm 2 \mathrm{bpm}$. Following to exchanging to combination drug, blood pressure, pulse rate, serum creatinine, protein excretion were not altered. Six month later, total cholesterol $(197 \pm 5$ to $183 \pm 3 \mathrm{mg} / \mathrm{dl}, \mathrm{p}<0.01)$ and triglyceride $(142 \pm 14$ to $129 \pm 10 \mathrm{mg} / \mathrm{dl}, \mathrm{p}<0.05$ ) were decreased and high density lipoprotein cholesterol $(53 \pm 3$ to $56 \pm 3 \mathrm{mg} / \mathrm{dl}, \mathrm{p}<0.05)$ was increased. Although central blood pressure was not altered significantly ( $124 \pm 4$ to $122 \pm 4 \mathrm{mmHg}$ ), FMD was slightly albeit significantly improved from $2.4 \pm 0.3$ to $2.7 \pm 0.3 \%$ (p $<0.05)$. There was a significant relationship between the improvement of FMD and decreases in total cholesterol $(\mathrm{R}=$ $-0.60, \mathrm{p}<0.01)$.

Conclusion: Our data indicate that combination drug is effective for CKD patients who have to take many pills, improving compliance. The present results suggest that atorvastatin refines endothelium function, partly due to improving lipid profiles.

\section{M-24 \\ Arterial Stiffness, Fatty Liver and the Presence of Coronary Artery Calcium in a Large Population Cohort}

Ki-Chul Sung

Division of Cardiology, Department of Medicine, Kangbuk Samsung Hospital, Sungkyunkwan University

School of Medicine, Seoul, Republic of Korea

Background: We tested whether fatty liver, brachialankle PWV (baPWV) and conventional cardiovascular risk factors were associated with a coronary artery calcium (CAC) score $>0$ (as a marker of the presence of early atherosclerosis) in a cohort of healthy Korean adults.

Methods: The study population consisted of individuals who underwent a comprehensive health examination in 2010 at Kangbuk Samsung Hospital, College of Medicine, Sungkyunkwan University in South Korea. The population included 7371 participants who had an assigned CAC score following coronary CT scanning and baPWV.

Results: Among the study subjects, $39.2 \%$ of the population had evidence of fatty liver by ultrasound and $4.6 \%$ of the population had evidence of CAC score $>0$. Among individuals with a CAC score $=0,38 \%$ of the individuals had fatty liver compared with $58 \%$ of the individuals with a CAC score $>0$. The individuals with a CAC score $>0$ also had higher blood pressure and had more metabolic abnormalities. The prevalence of CAC score $>0$ was increased according to baPWV quartiles and was higher in the fatty liver group in 
comparison with those without fatty liver. The OR for CAC score $>0$, after adjusting for clinical risk factors, showed a significant elevation with increasing quartiles of baPWV and the presence of fatty liver.

Conclusion: We showed that both fatty liver and baPWV are independently associated with the presence of CAC, a marker of preclinical atherosclerosis. These associations are independent of conventional risk factors and medical history.

\section{M-25 \\ In Korean Hypertension/Prehypertension Group Serum C-Reactive Protein is not Associated with Presence of Coronary Artery Calcium \\ Ki-Chul Sung \\ Division of Cardiology, Department of Medicine, Kangbuk Samsung Hospital, Sungkyunkwan University School of Medicine, Seoul, Republic of Korea}

Background: The levels of High-sensitive C-reactive protein (hs-CRP), a valid marker of cardiovascular disease (CVD), are higher in patients with subclinical atherosclerosis. Coronary artery calcium (CAC) scoring with cardiac computed tomography (CT) is a sensitive method to demonstrate the presence of pre-clinical atherosclerosis and the use of CAC scores may be also be useful in identifying individuals at increased risk of CVD. However, it is unclear that the association between hs-CRP and coronary calcium in hypertensive subjects.

Methods: Data were analysed from a South Korean occupational cohort of 12033 who underwent a cardiac CT estimation of CAC score and measurements of multiple cardiovascular risk factors. Patients were classified into three group; normal (4340 patients), prehypertension (5098 patients) and hypertension (2595 patients). Then prevalence and odds ratio (OR)s of presence of coronary calcium were calculated according to hs-CRP in each group.
Results: In normal and prehypertension group, the rate of presence of coronary calcium is higher in patients with high hs-CRP. After adjusting age, gender, past medical history, life style and obesity, the calculate ORs were 1.50 in normal group $(\mathrm{P}=0.022), 1.29$ in prehypertension group $(\mathrm{p}=0.089)$ and, $0.97(\mathrm{p}=0.82)$ in hypertension group.

Conclusion: High hs-CRP was associated with presence of coronary calcium in normal group. In such group of patients, hs-CRP predicts the presence of coronary calcium and provides information regarding CVD. However, it is not applied in hypertension/prehypertension group and the relationship between hs-CRP and the burden of coronary atherosclerosis is not clear in hypertensive subjects.

\section{M-26 \\ Acute Resistance Exercise Attenuates the Central Hemodynamic Response to Cold Pressor Test}

Eun Sun Yoon ${ }^{7}$, Soo Hyun Park', Yong Hee Lee', Jun Sung Kil', Ji Hun Yoon ${ }^{1}$, Hyun Jung Kim ${ }^{1}$, Jae Ho Park' ${ }^{1}$ Kevin Heffernan ${ }^{2}$, Bo Fernhall ${ }^{3}$, Sae Young Jae 1

${ }^{1}$ Department of Sport Science, University of Seoul, Seoul, South Korea; ${ }^{2}$ Department of Exercise Science, Syracuse University, Syracuse, NY, USA; ${ }^{3}$ Department of Kinesiology and Community Health, University of Illinois Urbana-Champaign, IL, USA

Background: Increased cardiovascular reactivity to sympathetic challenge is associated with increased risk of hypertension and cardiovascular disease. Acute aerobic exercise blunts stress related hemodynamic reactivity. Whether acute resistance exercise exerts a similar favorable hemodynamic effect is unknown. We tested the hypothesis that acute resistance exercise will attenuate central hemodynamic reactivity to a cold pressor test in healthy adults.

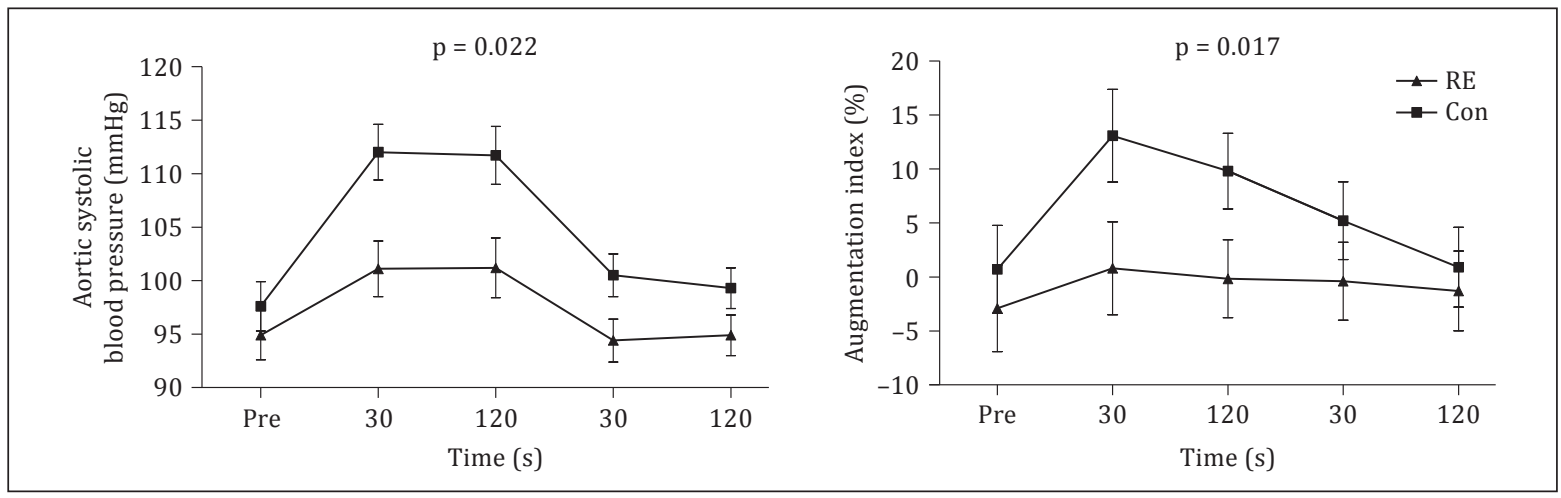

Fig. 1. Aortic systolic blood pressure and augmentation index responses to cold pressor test in the control (Con) and the resistance exercise (RE). Data presented as mean \pm SE. P values for interact effects (for Abstract M-26). 
Methods: Using a randomized cross-over trial, 15 healthy adults (male 9, female 6; age 26 \pm 5 yrs) completed two testing sessions (separated by one week). Sessions consisted of either a control condition (cold pressor test) or acute resistance exercise prior to cold pressor testing (2 set, 8 exercises, $40-60 \%$ of 1 RM for upper and lower extremity). Aortic blood pressure and pressure from wave reflections (augmentation index, AIx) were measured pre and $30 \mathrm{sec}$ and $120 \mathrm{sec}$ during a $3 \mathrm{~min}$ cold pressor test (ice water $4^{\circ} \mathrm{C}$ ), and $30 \mathrm{sec}$ and $120 \mathrm{sec}$ post-cold pressor testing, respectively.

Results: During the cold pressor test, there were significant increases in heart rate and rate pressure product $(\mathrm{p}<$ 0.05 ) in both group. However, acute resistance exercise attenuated increases in brachial systolic blood pressure, aortic systolic blood pressure, and AIx compared to the cold pressor testing alone (all $\mathrm{p}<0.05$ for interact effects).

Conclusion: These findings show that the cold pressor test significantly increases blood pressures and pressure from wave reflections in healthy adults, but an acute bout of resistance exercise attenuates the central hemodynamic response to the cold pressor test. Therefore, resistance exercise may have a favorable effect on cardiovascular reactivity to stress in healthy adults.

\section{M-27 \\ Changes of Potassium Channel Gene Expressions after Human Umbilical Mesenchymal Stem Cells Transfusion in Pulmonary Hypertension Rat Models}

Hyeryon Lee ${ }^{1}$, Kwan Chang Kim², Yoon Sun Yang ${ }^{3}$, Wonil $\mathrm{Oh}^{3}$, Soo Jin Choi ${ }^{3}$, Young Mi Hong ${ }^{1}$

${ }^{1}$ Department of Pediatrics, ${ }^{2}$ Department of Thoracic and Cardiovascular Surgery Ewha Womans University; ${ }^{3}$ Biomedical Research Institute, MEDIPOST, Co.3, Seoul, Korea

Background: Pulmonary arterial hypertension (PAH) is difficult to treat and is characterized by increased pulmonary arterial pressure, right heart failure and death. PAH has been shown to be refractory to most of the conventional pharmacological therapies. Human umbilical cord blood-derived mesenchymal stem cells (hUCB-MSCs) are regarded as an alternative source of bone marrow-derived mesenchymal stem cells. hUCB-MSCs have recently been studied for evaluation of their potential as a source of cell therapy. The purposes of this study were to investigate changes of pulmonary pathology, haemodynamics and gene expressions of $\mathrm{K}+$ channels, especially Kv1.7, Kir6.2.

Methods: The rats were grouped as follows: control group (C group), subcutaneous injection of saline; M group, subcutaneous injection of MCT (60 mg/kg); hUCB-MSCs transfusion (U group). hUCB-MSCs (3X106/mL/ $\mathrm{cm}^{2}$ ) were transfused by intraperitoneal injection 1 week after MCT injection.
Results: The mean right ventricular pressure (RVP) significantly decreased in the $U$ group compared with the M group in weeks 2 and 4 . RV weight and the ratio of $\mathrm{RH} /$ LH+septum significantly decreased in the U group compared to the $\mathrm{M}$ group. The number of muscular pulmonary arteriole significantly decreased in the U group compared with the $\mathrm{M}$ group in weeks 2 and 4. Medial wall thickness of the pulmonary arteriole significantly decreased in the $U$ group compared to the M group in week 2. Gene expressions of Kv1.7 was significantly decreased in the M group compared to the $\mathrm{C}$ group and increased in the $\mathrm{U}$ group compared to the $\mathrm{M}$ group in week 4. Gene expression of Kir6.2 was significantly increased in the $\mathrm{M}$ group and significantly decreased in the $\mathrm{U}$ group in week 4. Protein expression of Kv1.7 was significantly decreased in the $M$ group compared with the $C$ group and increased in the $\mathrm{U}$ group compared with the Mroup.

Conclusion: After hUCB-MSCs transfusion, there was improvement of RVH, mean RVP and survival rate. Kv1.7 gene expressions were decreased in the $M$ group and increased in the $U$ group in week 4. Additional research on the dose and frequency of hUCB-MSCs infusion is needed to determine the optimal parameters for PAH treatment.

\section{M-28 \\ Simultaneous Estimation of Flow Velocity and RBC Aggregation Using Ultrasound Speckle Analysis}

Eunseop Yeom, Sang Joon Lee

Center for Biofluid and Biomimic Research, Department of Mechanical Engineering, POSTECH, Pohang, South Korea

Background: Accurate estimation of hemodynamic parameters is important for early detection and diagnosis of cardiovascular diseases. Measurement of centerline velocity in a blood vessel dose not correctly offer wall shear stress information due to the non-Newtonian behavior of blood. Aggregation of red blood cells (RBCs) is a phenomenon that RBCs form rouleaux and rouleaux networks by aggregating and disaggregating in a reversible manner. The RBC aggregation intensifies the shear-thinning feature of blood flows. The variation of hemodynamic parameters affects the hemorheological parameters and vice versa.

Methods: The flow velocity and RBC aggregation were simultaneously estimated from ultrasound signals. The velocity distribution was obtained by applying cross-correlation algorithm to a pair of ultrasound images scattered by RBC aggregates. RBC aggregation was estimated by analyzing an echogenicity which is the ultrasound signal. To generate a pulsatile flow, a rat extracorporeal bypass model which connects a loop between the jugular vein and the abdominal aorta was used. A 35-MHz probe was used to detect the blood flows in the loop.

Results: The echogenicity profile varies largely according to the flow velocity, because it is highly dependent on hemo- 
dynamic condition. The pulsatile flow in the loop has a sharp acceleration, but with relatively gentle deceleration. The spatial distribution of RBC aggregation exhibits cyclic variations due to pulsatility of the flow. The centerline velocity has an inverse relationship with the echogenicity in the near-wall region. This implies that the high shear rate in the acceleration phase disaggregates the RBC aggregates and the decreased flow speed induces re-aggregation of RBCs. The velocity profile of the pulsatile flow is also systematically changed. In the systolic phase, it becomes sharper than the parabolic profile.

Conclusion: The velocity profile and RBC aggregation were measured simultaneously using the ultrasound speckle analysis. The rat extracorporeal model is useful to build up a realistic pulsatile flow without the change of blood properties. The echogenicity profile is changed according to the variation of hemodynamic conditions. The variation of bluntness seems to be closely related with RBC aggregation. These results would be helpful to understand the hemodynamic characteristics of pulsatile blood flows.

\section{M-29 \\ Association Between Carotid Intima Media Thickness and Pulse Wave Velocity in Middle-Aged Men}

\author{
Hyun Jeong Kim ${ }^{1}$, Eun Sun Yoon ${ }^{1}$, Soo Hyun Park', \\ Yong Hee Lee ${ }^{1}$, Kevin Heffernan ${ }^{2}$, Yoon-Ho Choi ${ }^{3}$, \\ Sae Young Jae ${ }^{1}$ \\ ${ }^{1}$ Department of Sport Science, University of Seoul, Seoul, \\ South Korea; ${ }^{2}$ Department of Exercise Science, Syracuse \\ University, Syracuse, NY, USA; ${ }^{3}$ Center for Health \\ Promotion, Samsung Medical Center, Sungkyunkwan \\ University School of Medicine, Seoul, South Korea
}

Background: Both carotid intima media thickening and arterial stiffening as early markers of atherosclerosis have been associated with cardiovascular events and mortality, but little and partly controversial information is available on the relationship between carotid intima media thickness and arterial stiffness.

Methods: We tested the cross-sectional and longitudinal associations of carotid intima media thickness with arterial stiffness in 107 middle aged men (mean age $52 \pm 6 \mathrm{yrs}$ ). We measured common carotid intima media thickness using an ultrasound machine and arterial stiffness was derived from brachial-ankle pulse wave velocity and brachial pulse pressure on two separate occasions. The changes in outcome variables were calculated as the difference between the first and second examinations (mean interval 402 \pm 122 days).

Results: Carotid intima media thickness was significantly correlated with age $(r=0.23, p=0.02)$, brachial-ankle pulse wave velocity $(r=0.27, p<0.01)$ and brachial pulse pressure $(\mathrm{r}=0.22, \mathrm{p}=0.02)$ at baseline. However, the change in carotid intima media thickness over 1 year was not significantly asso- ciated with the change in brachial-ankle pulse wave velocity $(\mathrm{r}=-0.13, \mathrm{p}=0.18)$ and brachial pulse pressure $(\mathrm{r}=-0.05, \mathrm{p}=$ 0.63 ) over that same time period.

Conclusion: These results demonstrate that carotid intima media thickening was not associated with arterial stiffening over time. Therefore, carotid intima media thickness and brachial-ankle pulse wave velocity may be different and independent stages of atherosclerotic process in middle aged men.

\section{M-30 \\ Longitudinal Assessment of Plaque Inflammation on Statin Therapy Evaluated by ${ }^{18}$ F-FDG PET/CT: A Prospective Interventional Study \\ Mineok Chang ${ }^{1}$, Joo Hyun O², Eun Ji Han' ${ }^{2}$, Eun-Ho Chu', Chun-Yeong Baek ${ }^{1}$, Byung-Hee Hwang ${ }^{1}$, Yoon-Seok Koh ${ }^{3}$, Pum-Joon Kim ${ }^{1}$, Ki-Bae Seung ${ }^{1}$, Sung Hoon Kim², Kiyuk Chang ${ }^{1}$ \\ ${ }^{1}$ Department of Cardiovascular Medicine, Seoul St. Mary's Hospital, The Catholic University of Korea, Seoul, Republic of Korea; ${ }^{2}$ Department of Radiology, The Catholic University of Korea, Seoul, Republic of Korea; ${ }^{3}$ Department of Cardiovascular Medicine, Uijeonbu St. Mary's Hospital, The Catholic University of Korea, Uijeongbu, Republic of Korea}

Background: Statin has beneficial effects in the primary and secondary prevention of cardiovascular disease by reducing plasma LDL-cholesterol (LDL-C) level and exerting pleiotropic actions on vessel walls, including anti-inflammation. No data exist on the longitudinal alterations in plaque inflammation by long-term statin therapy, while metabolic and inflammatory biomarkers are stabilized within a few months after initiation of statin therapy. The aim of this study is to investigate whether statin modulate plaque inflammation during 1 year of therapy by tracking and quantifying plaque inflammation with the use of serial ${ }^{18} \mathrm{~F}$-FDG PET/CT imaging.

Methods: This is a prospective, interventional study in statin-naïve stable angina patients with carotid plaques. After obtaining initial ${ }^{18} \mathrm{~F}$-FDG PET/CT of carotid arteries, 41 patients received atorvastatin $20 \mathrm{mg} /$ day, followed by a second ( $\mathrm{n}=28$; at 3 months of therapy) and a third FDG PET/CT ( $=9$; at 1 year of therapy). Primary observational endpoint was the percent change in arterial wall inflammation from baseline to second and second to third FDG PET/ CT assessed by the difference in target-to-background ratio (TBR) within the index carotid artery.

Results: Atorvastatin reduced mean LDL-C level by $36.4 \%$, under $70 \mathrm{mg} / \mathrm{dL}$, within 3 months ( $p=0.001$ ) and the level was lasted plateau for next 9 months $(\mathrm{p}=0.516)$. Through the study, mean HDL-C increased over $40 \mathrm{mg} / \mathrm{dL}$, by 3 months ( $p=0.041)$ and maintained it for the last months of therapy $(p=0.715)$ and mean serum hs-CRP levels showed a 
pattern of decrease within 3 months without clinical significance $(p=0.093)$. The percentage change of TBR in the index vessel showed continued reduction in plaque inflammation over 1 year of statin therapy: from baseline to 3 months $4.4 \%(p=0.015)$ and 3 months to 1 year $6.2 \%(p=0.009)$. In addition, the percentage change of TBR in bilateral carotid arteries and ascending aorta also showed continuous, significant reduction pattern.

Conclusion: Statin continues to exert anti-inflammatory effect on carotid atherosclerosis until 1 year even after achieving stable plasma LDL-C level at 3 months. ${ }^{18} \mathrm{~F}$-FDG PET/CT is a better tool to monitor the alterations in plaque inflammation than plasma lipid and inflammatory biomarkers.

\section{M-31 \\ Association between Cardio-Ankle Vascular Index (CAVI) and Diameter Change of Ascending Aorta during Systolic and Diastolic Phase on the Basis of Echocardiography \\ Sang Min Park, Hyun Hee Choi, Kyung Soon Hong, Byoung Ho Kim \\ Chuncheon Sacred Heart Hospital, Hallym University College of Medicine}

Background: Cardio-Ankle Vascular Index (CAVI) is a noble index of the overall stiffness of the artery from the origin of the aorta to ankle without influence of blood pressure at the time of measurement. We intended to investigate the echocardiographic aorta size difference between systole and diastole phase as a marker of arterial stiffness using simultaneous performing CAVI.

Methods: More than two hundred consecutive all comer patients $(62.3 \pm 14.9$ years old, $53 \%$ of male) undergoing transthoracic echocardiography during 6 months due to any cause were enrolled. We performed simultaneously CAVI to evaluate the degree of association between percent diameter change of ascending aorta (PDCA) during systolic and diastolic phase and CAVI.

Results: According to the age, the PDCA and average of both left and right CAVI increased while the average of both left and right Ankle-brachial index (ABI) decreased [Pearson coefficient: -0.156 ( $\mathrm{p}=0.027) ; 0.512(\mathrm{p}<0.001) ;-0.324(\mathrm{p}$ $<0.001)$ ]. The value of PDCA was higher in relative young age (Regression coefficient: $-0.086, p=0.005$ ) and female group $(1.49, p=0.035)$. However, it was not associated with the average value of CAVI $(0.325, p=0.179)$ the presence of hypertension, diabetes, dyslipidemia, and previous coronary or cerebrovascular history.

Conclusion: The value of PDCA was associated with age and sex and not associated with CAVI in this study. Large scaled trial for optimizing our findings would be warranted.

\section{M-32 \\ Protocatechuic Acid from Rubus Coreanus Miquel Suppresses the Expression of Adhesion Molecules TNF- $\alpha$-Stimulated Human Aortic Smooth Muscle Cells}

\author{
Hyunjung Koo ${ }^{7}$, Se-Chan Kang ${ }^{1}$, Seung Namkooing ${ }^{2}$, \\ Eun-Hwa Sohn ${ }^{3}$ \\ ${ }^{1}$ Department of Life Science, Gachon University, \\ Seongnam 461-701, Korea; ${ }^{2}$ Department of Physical \\ Therapy, Kangwon National University, Samcheok, \\ 245-710,Korea; ${ }^{3}$ Department of Herbal Medicine \\ Resource, Kangwon National University, Samcheok, \\ 245-710, Korea
}

Background: Activation of vascular smooth muscle cells (SMCs) contributes importantly to vascular inflammation and atherogenesis. The expression of adhesion molecules on aortic smooth muscle cells facilitates the accumulation of transmigrated leukocytes within the atherosclerotic vascular wall. Protocatechuic acid (PCA) from Rubi Fructus, the fruit of Rubus coreanus Miqel, is a polyphenolic compound which has been known that has antioxidant, anti-inflammaorty, antihyperglycemic, and anti-cancer properties.

Method: In the present study, we investigated the effect of PCA on the expression of adhesion molecules and focused on the transcription factor, NF- $\kappa \mathrm{B}$ in tumor necrosis factor $\alpha$ (TNF- $\alpha$ )-induced human aortic smooth muscle cells (HASMCs).

Result: It was shown that PCA significantly decreased mRNA and protein expression levels of ICAM-1 and VCAM-1 in TNF- $\alpha$-stimulated HASMCs and inhibited monocyte adhesion to the activated HASMCs. Moreover, PCA suppressed TNF- $\alpha$ induced NF-kB (p65) nuclear translocation.

Conclusion: Based on these results, we suggested that PCA has anti-inflammatory and anti-atherosclerotic properties through regulation of TNF- $\alpha$-induced expression of adhesion molecules by suppressing the NF-kB signaling pathway. 


\section{M-33 \\ Confounding Factors for Flow-mediated Vasodilation: FMD-Japan Study}

Takeshi Matsumoto', Yukihito Higashi, ${ }^{2,3}$, Masato Kajikawa ${ }^{1}$, Yumiko Iwamoto ${ }^{1}$, Shinsuke Mikami', Tatsuya Maruhashi ${ }^{1}$, Ayumu Nakashima3, Kensuke Noma 2,3, Hirofumi Tomiyama ${ }^{4}$, Bonpei Takase ${ }^{5}$, Akira Yamashina ${ }^{4}$, Yasuki Kihara ${ }^{7}$

${ }^{1}$ Department of Cardiovascular Medicine, Hiroshima University, ${ }^{2}$ Department of Cardiovascular Regeneration and Medicine, Research Center for Radiation Genome Medicine, Research Institute for Radiation Biology and Medicine, Hiroshima University, ${ }^{3}$ Division of Regeneration and Medicine, Hiroshima University Hospital, ${ }^{4}$ Second Department of Internal Medicine, Tokyo Medical University, ${ }^{5}$ Division of Biomedical Engineering, National Defense Medical College Research Insutitute

Background: Endothelial dysfunction is the initial step in the pathogenesis of atherosclerosis, resulting in cardiovascular outcomes. Recently, measurement of flow-mediated vasodilation (FMD) has been widely used to assess endothelial function in humans. The purpose of this study was to determine the relationships between FMD and cardiovascular risk factors, and evaluate confounding factors for measurement of FMD in a large general population.

Methods: A high-resolution ultrasonography, an automated edge tracking system, and an arm holding device were used to measure brachial artery diameter in response to reactive hyperemia (FMD, cuff inflated to $50 \mathrm{mmHg}$ above systolic blood pressure for $5 \mathrm{~min}$ ) in 5314 subjects ( $46 \pm 13 \mathrm{yr}$; 4131 men and 1183 women), including those with cardiovascular risk factor, such as hypertension, diabetes mellitus, and dyslipidemia, and those with cardiovascular disease, enrolled in FMD-Japan registry.

Results: Univariate regression analysis revealed that FMD correlated with age $(\mathrm{r}=-0.27, \mathrm{P}<0.001)$, body mass index $(r=-0.14, P<0.001)$, systolic blood pressure $(r=-0.18$, $P<0.001)$, diastolic blood pressure $(r=-0.13, P<0.001)$, total cholesterol $(\mathrm{r}=-0.07, \mathrm{P}<0.001)$, triglycerides $(\mathrm{r}=-0.10, \mathrm{P}$ $<0.001$ ), high-density lipoprotein cholesterol $(\mathrm{r}=0.06, \mathrm{P}<$ $0.001)$, low-density lipoprotein cholesterol $(\mathrm{r}=-0.04, \mathrm{P}=$ $0.01)$, glucose level $(\mathrm{r}=-0.14, \mathrm{P}<0.001), \mathrm{HbA1c}(\mathrm{r}=-0.14, \mathrm{P}$ $<0.001)$, and baseline brachial artery diameter $(\mathrm{r}=-0.43, \mathrm{P}<$ 0.001 ) as well as Framingham risk score, a cumulative cardiovascular risk index for heart attack $(\mathrm{r}=-0.29, \mathrm{P}<0.001)$. Multivariate analysis revealed that age, sex, BMI, systolic blood pressure, diabetes mellitus, smoking, and baseline brachial artery diameter were independent predictors of FMD

Conclusion: These findings suggest that FMD may be a marker of the grade of atherosclerosis and may be used as a surrogate marker of cardiovascular outcomes. Age, sex, body mass index, systolic blood pressure, diabetes mellitus, smoking, and particularly baseline brachial artery diameter are potential confounding factors in the measurement of FMD.

\section{M-34 \\ Experience of Brachial-Ankle Pulse Wave Velocity (baPWV) Measurement with Automated Device (VP-2000) for 4 Years in Cardiovascular Center of Dongguk University}

Deuk-Young Nah' ${ }^{1}$ Kwan Lee ${ }^{1}$, Jun-Ho Bae ${ }^{1}$, Jin-Wook Chung ${ }^{1}$, Ji-Hyun Kim², Yong-Seok Kim², Moo-Yong Rhee ${ }^{2}$, Young-Kwon Kim², Myoung-Mook Lee ${ }^{2}$

${ }^{1}$ Dongguk University College of Medicine, Gyeongju Hospital, Division of Cardiology, gyeongju, Republic of Korea; ${ }^{2}$ Dongguk University, Illsan Hospital, Cardiovascular Center, Goyang, Republic of Korea

Background: Even though carotid-femoral pulse wave velocity (PWV) is a standard method for measuring PWV. Recently, many investigators have been reported that the brachial-ankle PWV (baPWV) is also reliable measurement for arterial stiffness. We have experienced 4,311 measurement of baPWV between January 2007 and December 2010.

Methods: Patients with Diabetes Mellitus (DM) or Hypertension (HTN) (Group I, $\mathrm{n}=3,074,1,322$ men, $60 \pm 13$ years) and patients without coronary artery disease risk factors like a DM, HTN and Hypercholesterolemia (Group II, n = 1,237, 411 men, $50 \pm 16$ years) who undergone testing a baPWV were included. The measurement of the baPWV were performed in a quiet controlled $\mathrm{w}\left(22 \pm 1^{\circ} \mathrm{C}\right)$ in the supine position with using an automated device (VP-2000, Colin, Co. Ltd, Komaki, Japan).

Results : baPWV was positively correlated with age $(\mathrm{r}=$ 0.493, $\mathrm{p}<0.01)$, Systolic blood pressure $(\mathrm{r}=0.449, \mathrm{p}<0.01)$, Diastolic blood pressure $(r=0.269, \mathrm{p}<0.01)$, Heart rate $(\mathrm{r}=$ $0.250, \mathrm{p}<0.01)$ and hfPWV $(\mathrm{r}=0.670, \mathrm{p}<0.01)$. Multivariate linear regression analysis showed significant association of baPWV with age $(\beta=11.9, \mathrm{p}<0.01)$, systolic blood pressure $(\beta=7.7, p<0.01)$, BMI $(\beta=-14.7, p<0.01)$, heart rate $(\beta=$ $6.8, \mathrm{p}<0.01)$ and group of DM or HTN $(\beta=28.0, \mathrm{p}<0.01)$, $\mathrm{R} 2=0.520$ in all study subjects. The area under the ROC curve

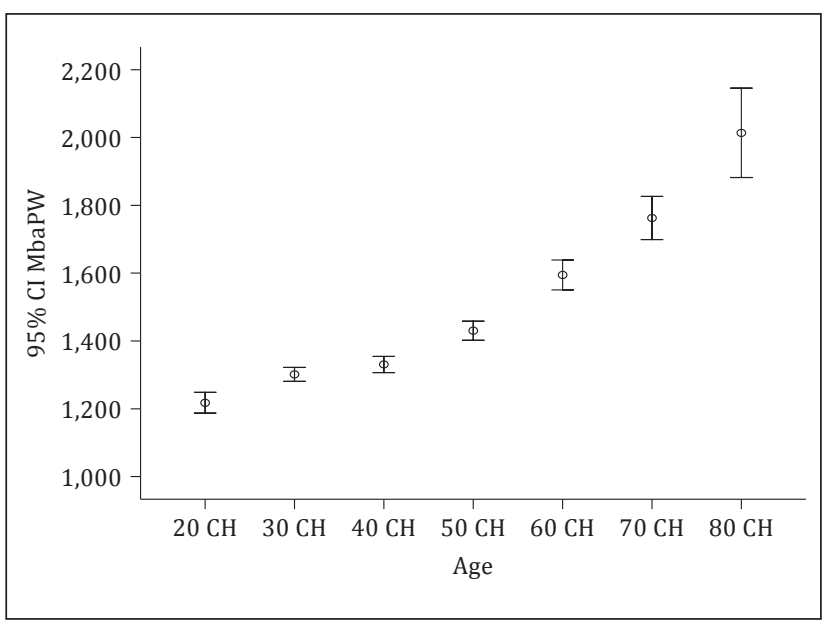

Fig. 1. (for Abstract M-34). 
Table 1. (for Abstract M-34)

\begin{tabular}{lll}
\hline & $\begin{array}{l}\text { Group I } \\
(\mathrm{n}=3,074)\end{array}$ & $\begin{array}{l}\text { Group II } \\
(\mathrm{n}=1,237)\end{array}$ \\
\hline Age (yrs) & $60 \pm 13^{*}$ & $50 \pm 16$ \\
Systolic blood pressure (mmHg) & $134 \pm 20^{*}$ & $126 \pm 19$ \\
Diastolic blood pressure (mmHg) & $79 \pm 13^{*}$ & $76 \pm 12$ \\
Heart rate (min) & $72 \pm 13^{*}$ & $68 \pm 12$ \\
baPWV (cm/sec) & $1,665 \pm 400^{*}$ & $1,439 \pm 318$ \\
hfPWV (cm/sec) & $1,052 \pm 254^{*}$ & $854 \pm 196$ \\
\hline
\end{tabular}

${ }^{*} \mathrm{p}<0.01$ by student t-test, hfPWV: heart-femoral pulse wave velocity.

Table 2. (for Abstract M-34)

\begin{tabular}{lll}
\hline & Group I $(\mathrm{n}=3,074)$ & $\begin{array}{l}\text { Group II } \\
(\mathrm{n}=1,237)\end{array}$ \\
\hline 3rd decade & $1352 \pm 234^{*}$ & $1218 \pm 178$ \\
4th decade & $1358 \pm 187^{*}$ & $1301 \pm 165$ \\
5th decade & $1412 \pm 285^{*}$ & $1320 \pm 217$ \\
6th decade & $1534 \pm 280^{*}$ & $1411 \pm 267$ \\
7th decade & $1724 \pm 386^{*}$ & $1555 \pm 371$ \\
8th decade & $1872 \pm 497^{*}$ & $1737 \pm 428$ \\
9th decade & $1969 \pm 673^{*}$ & $1930 \pm 550$ \\
\hline
\end{tabular}

${ }^{*} \mathrm{p}<0.01$ by student t-test

was 0.698 , and the cut-off value of baPWV for presence of coronary artery disease risk factors was $1470 \mathrm{~cm} / \mathrm{sec}$. at this cut-off value, sensitivity and specificity were $65 \%$ and $66 \%$. According to the decade, baPWV was shown in table 2.

This figure showed the baPWV according to the decade in Group II.

Conclusion: In this study, baPWV was higher in patients with DM and HTN than patients without DM and HTN and these results suggested that age, systolic blood pressure, BMI, Heart rate and patients with DM or HTN play a significant role in the arterial stiffness. 


\section{M-36}

Characterization and Quantification of Aortic Vortex Flow in Normal and Patients with Stroke Using Transesophageal Contrast Echocardiography

Jang-Won Son ${ }^{1}$, Chi Young Shim², Hyuk-Jae Chang ${ }^{2}$, Jong-Won Ha², Namsik Chung ${ }^{2}$, Helene Houle ${ }^{3}$, Mani A. Vannan ${ }^{4}$, Geu-Ru Hong ${ }^{2}$

${ }^{1}$ Division of Cardiology, Yeungnam University Medical Center, Daegu, Korea; ${ }^{2}$ Severance Cardiovascular Hospital, Yonsei University Health System, Seoul, South Korea; ${ }^{3}$ Siemens Medical Solution, Mountain View, CA; ${ }^{4}$ Piedmont Heart Institute Department of Cardiovascular Imaging, Atlanta, GA

Background: The flow in the aorta has specific geometry and forms vortex which is critical determinants of flow dynamics in aorta. Arteriosclerosis can alter blood flow pattern of aorta and may cause characteristic alteration of vortex. However, flow and vortex change in the aorta has not been studied yet. The aim of this study was to characterize aortic vortex flow pattern by transesophageal contrast echocardiography in normal and patients with stroke.

Methods: A total of 80 patients with stroke and 11 normal controls were recruited for this study. The 11 normal control subjects were designated as group 1 and 80 stroke patients were designated as group 2. All the subjects underwent contrast transesophageal echocardiography (TEE) and particle image velocimetry was used for assessing the aortic vortex flow. Both qualitative and quantitative analysis for vortex flow morphology, location, phasic variation and pulsatility were analyzed and compare between the groups.

Results: In group 1, multiple irregular shaped vortices with peripheral location were observed in descending thoracic aorta. In contrast, group 2 showed single, round merged and more centrally located vortex flow in aorta. In quantitative analysis of vortex, phasic variation of aortic vortex size is significantly decreased in group 2 compared with group 1 ( $58.8 \pm 23.5$ vs. $37.5 \pm 15.4, p=0.001)$. Vortex depth, which represents the location of major vortex in aorta, was significantly higher in group $1(0.64 \pm 0.15$ vs. $0.52 \pm 0.10$, $\mathrm{p}<0.001$ ). Vortex relative strength (VRS), which is the pulsatility parameter only for vortex itself, was significantly higher in group $2(0.30 \pm 0.97$ vs. $0.38 \pm 0.16, p=0.035)$. Figure shows example of aortic vortex flow pattern between two groups.

Conclusion: It was feasible to visualize and quantify the characteristic morphology and pulsatility of aorta vortex flow by contrast TEE, and the aortic vortex pattern was significantly different between normal and stroke patients. The vortex flow change in aorta can be surrogate marker for cardiovascular event and further prospective study is warranted.

\section{M-37 \\ Peripheral Arterial Tonometry is Useful in the Measurement of Endothelial Function in Korean Patients}

Weon Kim

Kyung Hee University Hospital

Background: A non-invasive flow-mediated vasodilation (FMD) predicted cardiovascular events in multi-ethnic, population-based studies. This study compares FMD and pulse amplitude tonometry (PAT), which is a novel technique that is less operator-dependent, in patients with coronary artery disease.

Methods: We analyzed the data from peripheral endothelial function assessments and clinical information from 80 consecutive patients with stable angina pectoris in our hospital, in a prospective observational study. All vasoactive medications were withheld for at least 24 hours before the measurements. Endothelial function was measured by a digital PAT, using the Endo-PAT, and by a standard FMD.

Results: In the overall study group (mean age $62 \pm 5$ years, $57 \%$ males) the mean FMD was $8.7 \pm 5.6 \%$ and the mean Endoscore, the value derived from the Endo-PAT, was $3.0 \pm 1$.7. There was a significant correlation between FMD and Endoscore in patients with stable angina pectoris $(\mathrm{R} 2=$ $0.36, \mathrm{p}<0.05$ ). A progressively higher quartile of FMD was associated with a higher Endoscore $(p<0.05)$. Also, there was a significant relationship between FMD and PAT in the diabetes group ( $R 2=0.29, \mathrm{p}<0.05)$, and in the non-diabetes group (R2 $=0.42, \mathrm{p}<0.05)$.

Conclusion: An assessment of PAT demonstrates patterns of abnormality, similar to those of FMD. These findings indicate that an analysis of PAT may be used to study vascular endothelial function in patients with coronary artery disease.

\section{M-38 \\ Association of Platelet Reactivity, Endothelial Function, and Inflammation in Patients with Stable Coronary Artery Disease}

Jong-Shin Woo

Kyung Hee University Hospital

Background: Platelets are actively involved in endothelial dysfunction and vascular atherosclerosis. We aimed to investigate the relationship between high on-treatment platelet reactivity (HOPR), systemic inflammation, and endothelial function of the peripheral arteries. In addition, we evaluated factors independently associated with ischemic cardiac events after elective percutaneous coronary intervention (PCI).

Methods: Ninety-one patients who planned to take follow-up angiography because of chest discomfort were 

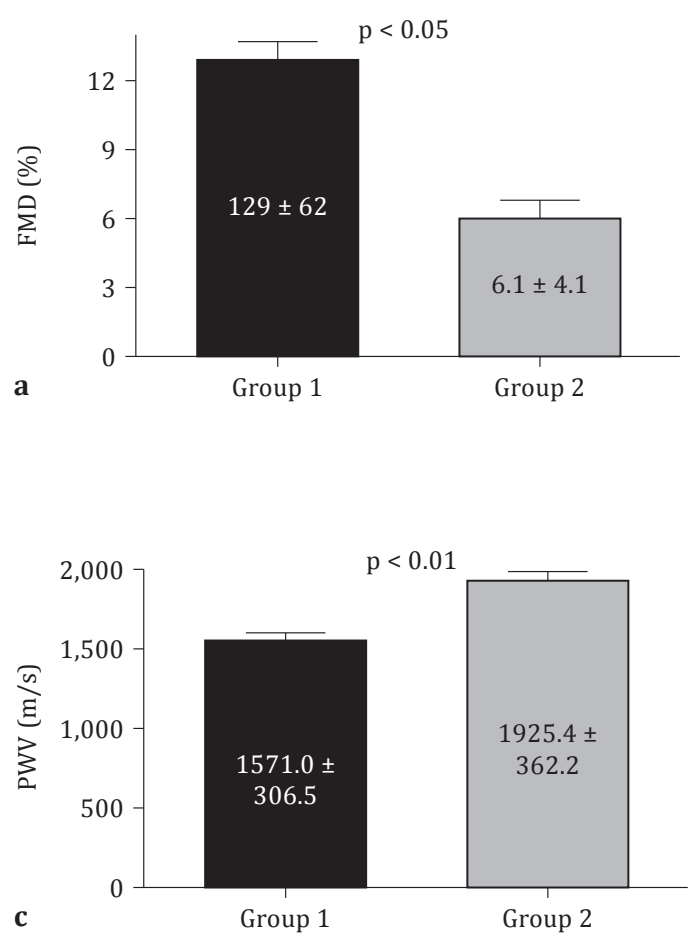
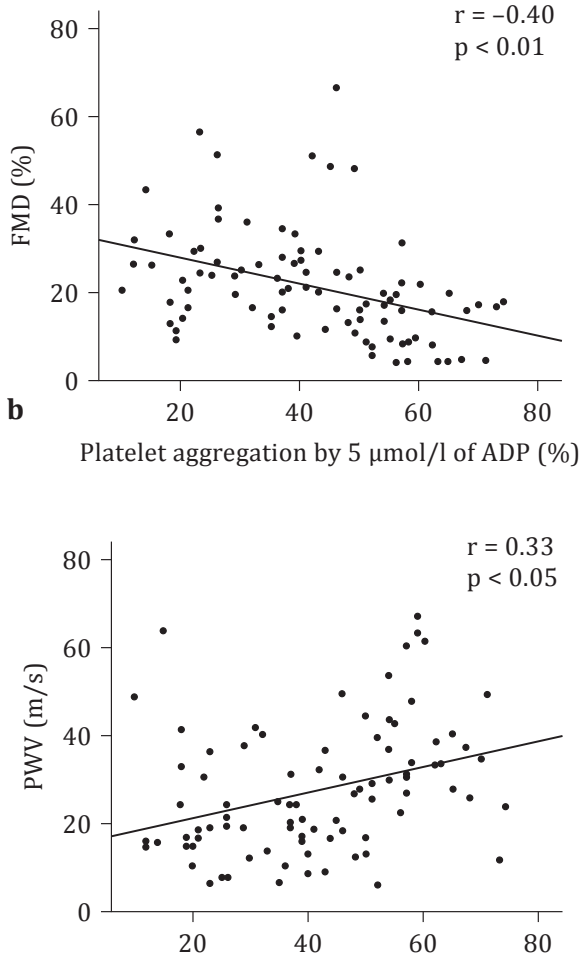

d Platelet aggregation by $5 \mu \mathrm{mol} / \mathrm{l}$ of ADP (\%)

Fig. 1. (for Abstract M-38).

consecutively enrolled. High-sensitivity C-reactive protein (hsCRP) was examined before coronary angiography. Platelet aggregation was assessed using light transmittance aggregometry [maximal platelet aggregation by $5 \mu \mathrm{mol} / \mathrm{L}$ of adenosine diphosphate $\leq 50 \%$ as group 1 and $>50 \%$ as group 2 (HOPR)]. Flow-mediated dilation (FMD) of the brachial artery and brachial-ankle pulse wave velocity (PWV) were assessed before angiography. Repeated PCI rates were evaluated.

Results: There were no differences in baseline characteristics and previous PCI data between group $1(\mathrm{n}=59)$ and $2(\mathrm{n}=32)$. When group 2 and 1 were compared, significantly lower FMD (6.1 \pm 4.1 vs $12.9 \pm 6.2 \%$, respectively) and higher PWV (1925.4 \pm 362.2 vs $1571.0 \pm 306.5$ ms, respectively) values were noted. Platelet reactivity negatively correlated with FMD and positively correlated with PWV. Significantly higher hsCRP levels were observed in group 2. Elevated hsCRP level, but not HOPR, was independently associated with repeated PCI.

Conclusion: Endothelial dysfunction and elevated hsCRP were significantly associated with HOPR in patients receiving chronic clopidogrel treatment.

\section{M-39 \\ Air Pollution Induces Endothelial Dysfunction via Oxidative Stress in Myocardial Injured Rats by Isoproterenol}

Weon Kim

Kyung Hee University Hospital

Background: Air pollution is an environmental health risk factor that is associated with increased cardiovascular morbidity and mortality. Here, we hypothesize that air pollution-mediated oxidative stress induces endothelial dysfunction.

Methods: Sprague Dawley Rats were injected with isoproterenol (ISO, $75 \mathrm{mg} / \mathrm{kg}$ ) subcutaneously, once daily, for 2 days. Two weeks after ISO administration, rats were exposed air pollutant in the specially designed car which measured the particulate air pollutants and exposed to animals in traffic areas in Seoul for 19 days, 4 hours a day. Aortic rings were embedded in Matrigel and photographed on day 5 with an inverted microscope. Data were constituted by counting the number of branch points in each image. To understand the role of air pollution to oxidative stress, aorta homogenates were used for in vitro ROS/RNS assay, which 
detects hydrogen peroxide, peroxylradical and peroxynitrite anion. Malondialdehyde (MDA) and nitric oxice (NO) levels were also assessed in aorta homogenates.

Results: ISO and concomitants with air pollution exposure impaired availabilities of tube formation in aorta. Total numbers of branch points were reduced by $64 \%$ and $79 \%$ in ISO-treated and air pollution exposure after ISOtreated groups respectively. ROS in aorta tissue was increased by ISO and ISO+AP by 1.3 and 1.45 fold as compared to no-treat group. In addition, MDA concentration was also increased by ISO and further pollutant exposure $(40.883 \pm 4.42$ in no-treat, $65.15 \pm 17.11$ in ISO and $74 \pm 12.76 \mathrm{nM} \mathrm{MDA} / \mathrm{mg}$ in ISO+AP). However total NO levels were not changed.

Conclusions: Air pollution impairs aorta endothelial function via ROS generation, with NO-independent manner in ISO-treated rats.

\section{$\mathrm{M}-40$ \\ Analysis of Risk Factors for Patients with Essential Hypertension Combining Coronary Heart Disease

\author{
Ze-Min Kuang ${ }^{1,2}$, Hong Yuan ${ }^{1,2}$, Zhi-Jun Huang ${ }^{1}$, Ying $\mathrm{Li}^{1}$
} \\ ${ }^{1}$ Hypertension Research Center of Human Province, Changsha 410013, China; ${ }^{2}$ Department of Cardiovascular, third Xiangya hospital, Central South University, Changsha 410013, China}

The aim of study is, 1) to investigate the characteristics of blood pressure and its relationship with the incidence of cardiovascular event in patients with essential hypertension (EH) combining coronary heart disease (CHD), 2) to identify the relationship between serum uric acid (UA) and coronary heart disease as well as hypertension, and 3) to explore the prediction of the combination serum uric acid level and left venticualr mass index (LVMI) in occurrence of cardiovascular events.

Background: Effective prevention of cardiovascular disease (CVD) requires the early detection and correction of predisposing conditions and risk factors in susceptible patients. Hypertension is a common risk factor for CVD, and the cardiovascular prognosis in patients with hypertension depends not only on the level of blood pressure (BP), but also on the presence of associated risk factors. Hyperuricemia is frequently encountered in hypertensive patients. Several large epidemiologic studies have identified an association between increased serum uric acid (UA) and cardiovascular risk in the general population and among patients with hypertension. On the other hand, the level of serum UA is affected by or linked to many factors, such as obesity, insulin resistance, dyslipidemia, and hypertension, all of which are also associated with left ventricular hypertrophy (LVH). In hypertension, LVH is initially a compensatory process against abnormal loading conditions, but it is also the first step toward the development of overt clinical disease, such as CVD. In essential hypertension, the risk of future CVD complications is higher in patients with LVH on echocardiography than in those with normal left ventricular (LV) mass. Thus, assessment of LV mass by echocardiography is a well-established procedure to estimate the risk of CVD in hypertensives.

Methods: The relationships between the coronary angiography diagnosis and clinical data were analyzed with 546 essential-hypertensive patients who received coronary angiography. Blood pressure in all patients with EH or EH combining CHD was measured with ambulatory blood pressure monitoring (ABPM), patients were followed up for six months. The correlativity between parameters of ambulatory blood pressure and the risk of cardiovascular events were analyzed. All patients underwent coronary angiography. The severity of coronary artery stenosis was evaluated by the number of impaired arteries and Gensini scores. The levels of fasting plasma glucose (FPG), triglyeride (TG), total cholesterol (TC), low density lipoprotein-cholesterol (LDL-C), high density lipoprotein-cholesterol (HDL-C), serum uric acid (SUA), and serum creatinine (SCr) were measured by HITACHI 7080 automatic biochemistry analyzer in 546 patients with coronary heart disease and hypertension.

Results: The incidence of cardiovascular event was higher in patients with essential hypertension (EH) combining coronary heart disease (CHD) than that in patients with EH. Age, Gensini scores, $24 \mathrm{~h}$ systolic blood pressure (SBP), day SBP, night SBP, 24 h pulse pressure (PP), day PP and night PP were higher in patients with $\mathrm{EH}$ combining CHD than that in patients with only $\mathrm{EH}$. In the Logistic multivariate analysis, age, Gensini score, $24 \mathrm{~h} \mathrm{SBP}$ and night PP were the best predictor for the incidence of cardiovascular event in patients with EH combining CHD. Patients with essential hypertension combining coronary heart disease had strongly higher concentration of UA compared to that of patients with essential hypertention solely. The ratios of hyperuricemia concomitant hypercholesterolemia and hypertriglyceridemia were significantly elevated than those of non-hyperuricemia concomitant hypercholesteremia and hypertriglyceridemia.

Conclusion: Male, high salt intake, hypercholesterolemia and typic angina pectoris may be the predictors of the occurrence of cadiovascular disease for patients with essential hypertension combining caoronary heart disease. Age, Gensini scores, $24 \mathrm{~h}$ SBP and night PP were the risk factors for the incidence of cardiovascular event in patients with EH combining CHD. To control these factors might reduce the incidence of cardiovascular events. Hyperuricemia and LVMI has relationship with many risk factors for $\mathrm{CHD}$ and is an independent risk factor for incidence of hypertension with CHD. 


\section{M-41 \\ Thoracic Aortic Circumferential Strain and Atherosclerosis in Patients with Cerebral Infarction

\author{
Mi-Seung Shin, Jin-Kyu Kang, Hyun Mi Park, \\ Jeonggeun Moon, Wook-Jin Chung, Tae Hoon Ahn, \\ Eak Kyun Shin \\ Department of Cardiology, Gachon University Gil \\ Hospital
}

Background: Crebral infarction is related with atherosclerosis. Circumferential carotid artery strain can be used as a screening tool for subclinical atherosclerosis. The purpose of this study was to evaluate the relationship between thoracic aortic circumferential strain and atherosclerosis in patients with cerebral infarction.

Methods: Seventy two patients with cerebral infarction who had examined both transthoracic and transesophageal echocardiography for cerebral infarction and 49 controls were enrolled. Atrial fibrillation, patent foramen ovale, severe valvular heart disease and left atrial thrombus were excluded. After the exclusion, 43 patients with cerebral infarction and 31 controls were included. We analyzed left ventricular systolic and diastolic function, and global circumferential strain of descending thoracic aorta (DTA). Thoracic aortic circumferential strain was obtained from the ratio of change in circular length during the cardiac cycle. Atherosclerotic change of DTA and aortic arch was graded by 5 grading system.

Results: Global circumferential strain of DTA showed significant positive correlation with thoracic aortic luminal distensibility $(\mathrm{r}=0.46, \mathrm{p}<0.01)$ and fractional area change $(\mathrm{r}=0.65, \mathrm{p}<0.001)$ in patients with cerebral infarction. Global circumferential strain of DTA showed significant negative correlation with $\beta$-stiffness of thoracic aorta $(r=-0.47, p=$ 0.001 ) in patients with cerebral infarction. Also global circumferential strain of DTA showed positive correlation with mitral annular early diastolic tissue velocity $(r=0.49, \mathrm{p}=0.001)$ and E/A ratio ( $\mathrm{r}=0.45, \mathrm{p}=0.003)$, and showed negative correlation with the grade of atherosclerotic change $(r=-0.41, p=$ $0.006)$ in patients with cerebral infarction. However, global circumferential strain of DTA showed additional positive correlation with left ventricular ejection fraction $(r=0.36, p=$ 0.043 ), and showed no significant correlation with the grade of atherosclerotic change in normal controls.

Conclusion: Thoracic aortic circumferential strain was well correlated with mechanical indices reflecting aortic stiffness and cardiac diastolic function. Furthermore, Thoracic aortic circumferential strain can be used as an objective and quantitative marker of atherosclerosis in patients with cerebral infarction.

\section{M-42 \\ Supplementation of a Palm Oil Based Tocotrienol Rich Vitamin E Preparation in Young Smokers and Its Effects on Central Aortic Systolic Pressure}

\author{
Azli Shahril Othman, Abdul Rashid Abdul Rahman \\ Cyberjaya University College of Medical Sciences
}

Background: The effects of tocotrienol on cardiovascular health have been previously assessed and discussed. In this study, we looked at the effects of a palm oil based, tocotrienol-rich vitamin E preparation on central aortic systolic pressure (CASP) and augmentation index (AIx), which are now considered robust predictors of cardiovascular risk and clinical outcome, on a population of young cigarette smokers with no associated co-morbidities.

Methods: A total of 42 young, healthy, male smokers (mean age 23.19 \pm 3.08 ) and 48 young, healthy, male nonsmokers (mean age 22.58 \pm 3.04 ), randomized into four groups i.e. smokers on intervention, smokers on placebo, non-smokers on intervention and non-smokers on placebo, completed the three-month study. The commercially available Tocovid SupraBio (Hovid, Ipoh, Malaysia) on a $200 \mathrm{mg}$ once daily dosing were used as the intervention. CASP, AIx and brachial blood pressure values, obtained using BPro (Healthstats, Singapore), were recorded during the baseline visit, and again at the end of study. Subjects with associated cardiovascular comorbidities, known food or drug allergies, or were on medications or supplements, were excluded.

Results: There was a small, non-significant reduction in CASP in the smokers on intervention group (104.60 \pm 4.89 to $103.25 \pm 4.90, P=0.33$ ). Similarly there were no significant differences between baseline and end-of study values for the other parameters of interest i.e. AIx, brachial systolic pressure and brachial diastolic pressure. Following statistical adjustment of data for age, heart rate, height, weight, total cholesterol and glucose, the mean differences of all parameters of interest remained statistically insignificant.

Conclusion: Supplementation of tocotrienol-rich vitamin E in young, healthy, cigarette smokers yielded no statistically significant improvement in CASP and AIx. However the trend of CASP improvement in young smokers supplemented with tocotrienol-rich vitamin $\mathrm{E}$ is promising and may warrant larger trials/dose optimisation studies. 


\section{M-43 \\ Different Patterns of Aortic Stiffness and Vasodilation Caused by $\beta$-Blockers in Spontaneously Hypertensive Rats}

Wu-Gang Zhou, Ke Zhang, Dong-Rui Chen, Wei-Li Shen, Ding-Liang Zhu, Ping-Jin Gao

Departement of Hypertension, RuiJin Hospital, Shanghai Key Lab of Vascular Biology, Shanghai Institute of Hypertension, Shanghai Jiao Tong University, School of Medicine, Shanghai,China

Backgroud: Compared with conventional $\beta$-blockers, it is not clear whether vasodilating $\beta$-blockers have beneficial effects on arterial stiffness, including decreased pressure wave reflection, and improvement of vascular wall components. In this study, we investigated different parameters of vascular function in spontaneously hypertensive rats treated with $\beta$-blockers, either with or without vasodilator properties, by determining their biochemical mechanisms. Furthermore, another aim of the present study was to evaluate whether $\beta$-blockers that stimulate NO production are more advantageous as anti-hypertensive agents when compared to a $\beta$-blockers, with respect to vasodilating $\beta$-blockers.

Methods: Spontaneously hypertensive rats (SHRs) were treated with metoprolol $\left(200 \mathrm{mg} \bullet \mathrm{kg}^{-1} \bullet \mathrm{d}^{-1}\right)$, arotinolol (30 $\left.\mathrm{mg} \bullet \mathrm{kg}^{-1} \bullet \mathrm{d}^{-1}\right)$, and nebivolol $\left(15 \mathrm{mg} \bullet \mathrm{kg}^{-1} \bullet \mathrm{d}^{-1}\right.$ ) for 8 weeks. Age-matched Wistar-Kyoto rats and untreated SHRs were used as controls. Central arterial pressure and pulse wave velocity were evaluated via catheter pressure transducers. The rings of rat aorta, and renal and mesenteric arteries were evaluated by isometric force recording. Collagen was assessed by immunohistochemistry, while endothelial nitric oxide synthase (eNOS) and eNOS phosphorylation (p-eNOS) were analyzed by western blotting. Nitric oxide and reactive oxygen species were measured in human aortic endothelial cells by using fluorescent probes.

Results: Nebivolol and arotinolol, rather than metoprolol, markedly decreased central arterial pressure and pulse wave velocity at week 8 , and compared to metoprolol, both nebivolol and arotinolol obviously increased vasorelaxation, aortic vasorelaxation to acetylcholine and p-eNOS/eNOS, and the effects of nebivolol were more pronounced. Besides, aortic collagen depositions in the nebivolol and arotinolol groups were reduced compared with those in the metoprolol group or untreated SHRs. Nebivolol and arotinolol produced more nitric oxide and had higher oxygen radical-scavenging capacities compared to metoprolol in the endothelial cells. Intriguingly, a potassium channel inhibitor (4-aminopyridine) caused a significant reduction in artinolol-induced vasorelaxation, but not nebivolol.

Conclusions: Vasodilating $\beta$-blockers with nitric oxide production may cause increased reduction of arterial stiffness via different mechanisms.

\section{M-44 \\ Iron Metabolism Disorder May Contribute to Hypoxia-Induced Pulmonary Hypertension}

Li Ying ${ }^{1}$, Yuan Hong ${ }^{1}$, Li Xiao-Hui ${ }^{2}$

${ }^{1}$ The Third Xiangya Hospital, Central South University, Changsha, China; ${ }^{2}$ Department of Pharmacology, School of Pharmaceutical Science, Central South University, Changsha, China

Background: Iron supplement is efficient to inhibit the increase of pulmonary arterial systolic pressure (PASP) induced by hypoxia. And recently, iron deficiency is normally observed in idiopathic pulmonary arterial hypertension (IPAH) patients, while the situation of iron metabolism and its regulatory mechanism under hypoxic pulmonary hypertension (HPH) isseldom known. The aim of is study is to observe is there exists iron metabolism disorder and discuss the possible mechanism in HPH rat.

Methods: HPH rats was induced by 4-weeks hypoxia. Beside blood regular test, blood samples were collected for determination of several factors related to iron metabolism including iron, ferritin, transferritin and hepcidin, which is synthesised in liver and plays a key role in inhibiting iron absorption, release and storage. Furthermore, RNA and protein were extracted from liver tissues to evaluate the transcriptional level of hepcidin and protein expression of the upstream regulator of hepcidin, BMP6.

Results: Iron concentration $(12.5 \pm 2.41$ vs $5.78 \pm 2.86$, $\mathrm{P}=0.033$ ) was significantly decreased in HPH rats, while the plasma level of transferritin $(1.6 \pm 0.59$ vs $5.78 \pm 2.86, \mathrm{P}=$ $0.012)$ and hepcidin $(1.6 \pm 0.59$ vs $5.78 \pm 2.86, \mathrm{P}=0.048)$ were increased. Plasma level of ferritin was also decreased but the change is not significant $(1.6 \pm 0.59$ vs $5.78 \pm 2.86, \mathrm{P}=0.051)$. WBC $(1.6 \pm 0.59$ vs $5.78 \pm 2.86,=0.013)$ and MCV $(57.58 \pm 2.39$ vs $61.28 \pm 2.01, \mathrm{P}=0.029)$ were decreased in HPH rats showed from blood regular test. Q-PCR and western blot experiments showed that hepcidin mRNA level and BMP6 protein level were both significantly increased in liver.

Conclusion: Disorder of iron metabolism generally exists in $\mathrm{HPH}$ rats and an up-regulatedBMP6/hepcidin signalling pathway in liver may contribute to this progress.

\section{M-45 \\ Ambulatory Blood Pressure Variability: An Independent Determinant of Arterial Stiffness \\ Moo-Yong Rhee, Sungha Park, Ji-Hyun Kim \\ Dongguk University Ilsan Hospital, Yonsei University}

Objectives: There are many factors associated with arterial stiffness. Among them, blood pressure (BP) is a major determinant of arterial stiffness. The present study evaluated the effect of ambulatory blood pressure variability (ABPV) on arterial stiffness. 
Methods: Brachial-ankle pulse wave velocity (baPWV), augmentation index corrected for heart rate (AI75) and $24 \mathrm{Hr}$ ABP were measured from 606 subjects $(47.2 \pm 9.3$ years, range 20-70 years) who were randomly selected from general population (NCT01438619) and volunteers (NCT01237717), excluding subjects taking antihypertensive medications. Standard deviation and average real variability of 24 hour, daytime and nighttime period were calculated as ABPV.

Results: All ABPV parameters were well correlated with baPWV. ABPV of $24 \mathrm{Hr}$ systolic BP and daytime BP were significantly correlated with AI75. Partial correlation and multivariate regression analysis adjusted with age, gender, body mass index, smoking status, presence of diabetes, level of LDL, and heart rate of each time period showed significant association of $24 \mathrm{Hr}$ and daytime ABPV with baPWV and AI75. Nighttime ABPV was not associated with baPWV and AI75. Although the association between ABPV and baPWV was not persistent after further adjustment with ambulatory BP of each time period, the association between ABPV and AI75 was persistent. Further analysis for quartiles of ABPV also showed significant linear trend association of ABPV quartiles with baPWV and AI75. Most of highest quartile showed significantly higher value of baPWV and AI75, compared to the lowest quartile. Among the ABPV variables, $24 \mathrm{Hr}$ systolic $\mathrm{ABPV}$ has the strongest association with baPWV and AI75.

Conclusion: The present study revealed ABPV as an independent determinant of arterial stiffness.

\section{M-46 \\ Urine Albumin Creatinine Ratio Could Predict Coronary Heart Disease Risk in Real World}

Seoung-Pyo Hong Young-Soo Lee, Yeun-Woong Park, Kyung-Ryun Bae, Jin-Bae Lee, Jae-Kean Ryu, Ji-Yong Choi, Kee-Sik Kim, Sung-Gug Chang

Department of Cardiology, Daegu Catholic University Medical Center

Background: Urine albumin creatinine ratio (UACR) is known as maker of subclinical target organ damage. We wonder if UACR can help us to assess cardiovascular risk using Framingham coronary heart disease (CHD) risk score (FRS) in real world populations.

Methods: We enrolled the 1177 patients (30-74 years, mean age 57 years, male $49.5 \%$ ) visiting outpatient clinic from March 2009 to Feb. 2012. The patients with coronary revascularization, stroke and heart failure were excluded. The FRS was estimated using age, sex, smoking, systolic blood pressure, total and HDL cholesterol. Also, UACR, use of angiotensin converting enzyme inhibitor (ACE inhibitor) and statin and echocardiographic parameters like left ventricular (LV) ejection fraction, LV mass index (LVMI) and left atrial volume index (LAVI) were assessed. The absolute 10-year CHD risk was classified as relative low risk $(<15 \%)$ and relative high risk ( $\geq 15 \%)$.
Results: The 268 patients (male 48.1\%) with relative high risk were significantly older than the 909 patients with relative low risk (male 54.5\%). The patients with relative high risk had significantly higher UACR than the patients with relative low risk $(0.137 \pm 0.383$ vs. $0.019 \pm 0.034 \mathrm{mg} / \mathrm{mg}$, respectively). Also, LVMI and LAVI were significantly larger in patients with relative high risk. However, LVEF and use of ACE inhibitor and statin was similar between both groups. The cutoff value of UACR for predicting the relative high risk was $0.0125 \mathrm{mg} / \mathrm{mg}$ with $64 \%$ of sensitivity and $62 \%$ of specificity.

Conclusions: UACR might be significantly associated with coronary heart disease risk. Moreover UACR could help us to discriminate the patients with high coronary heart disease risk in real world populations.

\section{M-47 \\ Severity of Ophthalmic Artery Flow Might be Useful Predictor for Coronary and Carotid Artherosclerosis}

Seoung-Pyo Hong, Yeun-Woong Park, Seung-Woon Jeon, Kyung-Ryun Bae, Young-Soo Lee, Jin-Bae Lee, Jae-Kean Ryu, Ji-Yong Choi, Kee-Sik Kim, Sung-Gug Chang

Department of Cardiology, Daegu Catholic University Medical Center

Background: Doppler imaging of the Ophthalmic artery (OA) have provided new insights into various vascular disorders including carotid stenosis, coronary artery disease (CAD). The aim of the investigation was to assess the clinical usefulness of doppler waveform indices of OA for estimating the severity of carotid and coronary artherosclerosis.

Methods : The study was a retrospective analysis of the findings in 140 patients performed coronary angiography (CA) for evaluation of CAD between July 2010 and October 2011 at our hospital. CAD was defined as confirmed above $50 \%$ obstructive of major coronary arteries in CA. For evaluation of $\mathrm{OA}$ blood flow velocity and carotid maximum intimamedia thickness (c-IMT) and carotid plaque, we performed the pulsed Doppler of OA and carotid ultrasound before CA.

Results: The patients were 90 men and 50 women with mean age of 60 years. CAD was 87 patients $(62.1 \%)$ and carotid plaque was 39 patients (27.9\%). Mean systolic velocity (MSV)/ mean systolic velocity (MDV) and resistance index (RI) was the independent predictor for CAD after adjustment for CAD-associated factors. (MSV/MDV > 2.0, OR 4.3, 95\%CI 1.6-11.1, $\mathrm{p}=0.003, \mathrm{RI}>0.71$, OR 2.6, 95\%Cl 1.1-6.4). Also, MSV/MDV (OR 3.4, 95\%CI 1.2-9.4, p = 0.021) and RI (OR 2.5, $95 \% \mathrm{Cl} 1.0-6.4, \mathrm{p}=0.049$ ) were the independent predictor for carotid plaque after adjustment for CAD-associated factors. And, MSV/MDV ( $\mathrm{r}=0.475, \mathrm{p}<0.001)$ and RI ( $\mathrm{r}=0.326, \mathrm{p}<$ 0.001 ) were a moderate positive correlation with c-IMT. After adjustment for CAD-associated factors, only MSV/MDV ( $\mathrm{r}=$ $0.380, p=0.046$ ) was a moderate positive correlation with c-IMT. 
Conclusion: Severity of ophthalmic artery flow might be useful predictor for CAD, carotid plaque and carotid artherosclerosis.

\section{M-48 \\ Intima-Media Thickness of Brachial Artery is a Useful Marker of Atherosclerosis}

Tatsuya Maruhashi', Yumiko Iwamoto ${ }^{7}$, Yuichi Fujii', Naomi Idei', Noritaka Fujimura', Shinsuke Mikami', Masato Kajikawa', Takeshi Matsumoto?, Yasuki kihara', Kazuaki Chayama ${ }^{2}$, Kensuke Noma 3,4, Ayumu Nakashima ${ }^{4}$, Yukihito Higashi3,4

${ }^{1}$ Department of Cardiovascular Medicine, ${ }^{2}$ Department of Medicine and Molecular Science, Graduate School of Biomedical Sciences, ${ }^{3}$ Department of Cardiovascular Regeneration and Medicine, Research Center for Radiation Genome Medicine, Research Institute for Radiation Biology and Medicine, Hiroshima University, ${ }^{4}$ Division of Regeneration and Medicine, Hiroshima University Hospital, Hiroshima, Japan

Background:Cardiovascular diseases are associated with impaired flow-mediated vasodilation (FMD) and increase in carotid intima-media thickness (IMT). Both FMD and IMT are independent predictors for cardiovascular outcomes. When measuring FMD and nitroglycerine-induced vasodilation in the brachial artery, IMT can also be simultaneously assessed in the same brachial artery. The purpose of this study was to determine the relationships between IMT of the brachial artery, vascular function, and cardiovascular risk factors.

Methods: We measured brachial IMT, FMD, and nitroglycerine-induced vasodilation by ultrasound in 388 subjects who underwent health examination (mean age, $45 \pm 22$ years; age range, 19-86), including patients with cardiovascular diseases.

Results: Univariate regression analysis revealed that brachial IMT significantly correlated with age $(\mathrm{r}=0.71$; $\mathrm{P}<$ $0.001)$, body mass index $(\mathrm{r}=0.27 ; \mathrm{P}<0.001)$, systolic blood pressure $(r=0.40 ; P<0.001)$, diastolic blood pressure $(r=$ 0.31 ; $\mathrm{P}<0.001)$, heart rate $(\mathrm{r}=0.15 ; \mathrm{P}=0.002)$, glucose level $(\mathrm{r}=0.18 ; \mathrm{P}=0.01)$, and smoking pack-years $(\mathrm{r}=0.42$; $\mathrm{P}<0.001$ ), as well as Framingham risk score, a cumulative cardiovascular risk index for heart attack $(r=0.49$; $\mathrm{P}<0.001)$. FMD and nitroglycerine-induced vasodilation were inversely associated with brachial IMT ( $r=-0.39, \mathrm{P}<0.001 ; \mathrm{r}=-0.32$, $\mathrm{P}<0.001$, respectively). In addition, there was a significant relationship between brachial IMT and carotid IMT $(r=0.58$; $P<0.001)$. Multivariate analysis revealed that age, sex, hypertension, and brachial artery diameter were independent predictors of brachial IMT.

Conclusion: These findings suggest that brachial IMT may be a marker of the grade of atherosclerosis and may be used as a marker of vascular function, providing additive information for stratifying subjects with cardiovascular risk factors.

\section{M-49 \\ The Change of the Blood Pressure Depending on the Exercise Type in the Hypertensive Population With or Without Medication}

Juneyoung Yoon', Juyong Lee ${ }^{2}$, Sun Hyo Park', Hui-Kyoung Sun ${ }^{1}$, Moon Chan Choi ${ }^{1}$, Sang Dae Choi', Dae-Hee Shin ${ }^{3}$

${ }^{1}$ Department of Internal Medicine, Kurosungsim Hospital, South Korea; ${ }^{2}$ Department of Cardiology, St Elizabeths Medical Center of Boston, USA; ${ }^{3}$ Gangneung Asan Hospital, University of Ulsan College of Medicine, Gangneung, South Korea

Background: Healthy lifestyle modification (LSM) such as exercise or weight loss is an important first step for lowering blood pressure (BP) in hypertension. Some people who found themselves have high BP just tend to try lower BP with some LSM to avoid or delay taking medication initially. However, only lifestyle modification is difficult to predict the outcome. The aim of the study was to show the change of mean systolic blood pressure depending on the exercise patterns in the hypertensive patients with or without medication.

Methods: Data were from three rounds of nationally representative cross-sectional surveys for Korean men and women adult. We selected the adult target population 6646 patients who already diagnosed HTN or whose systolic BP (SBP) measured twice $>=140$ or diastolic blood BP (DBP) $>=$ 90. In order to see difference of the exercise effect independent of the medication, we divided 3802 patients with drug treated group (TG) and 2843 patients with non-drug treated group (NTG) of hypertension and compared the mean systolic blood pressure depending on the types of exercise. We calculated the mean SBP with general linear model using SPSS 18.

Results: The number of hypertension patient were 3803 in TG (57.2\%) and 2843 in NTG (42.8\%). The distribution of the type of exercise in TG and NTG revealed 4 types including no-exercise/walking exercise /intense exercise/ both walking and intense exercise: $24.0 / 15.6 / 4.2 / 5.6 \%$ in TG, 24.8/18.4/3.3/4.1\% in NTG, respectively. The mean SBP of the TG and NTG was 132.4 and $138.2 \mathrm{mmHg}$, respectively. The mean SBP of the TG and NTG depending on the type of exercise was 132.6 and $138.0 \mathrm{mmHg}$ at no exercise, 133.3 and $139.7 \mathrm{mmHg}$ at walking, 132.4 and $138.9 \mathrm{mmHg}$ at intense exercise, 131.3 and $136.3 \mathrm{mmHg}$ at walking/intense exercise both, respectively $(p<0.000)$. The mean DBP of the TG and NTG was 81.2 and $92.0 \mathrm{mmHg}$, respectively. The mean DBP of the TG and NTG depending on the type of exercise was 80.9 and $91.9 \mathrm{mmHg}$ at no exercise, 80.7 and $92 \mathrm{mmHg}$ at walking, 83.5 and $92.9 \mathrm{mmHg}$ at intense exercise, 83.1 and $92.1 \mathrm{mmHg}$ at walking/intense exercise both, respectively $(\mathrm{p}<0.000)$.

Conclusion: In both group, the walking and intense exercise showed the best systolic blood pressure lowering effect. The walking/intense exercise group was the only group that has systolic BP close to $135 \mathrm{mmHg}$ in NTG. However, exercise did not show DBP lowering effect regardless of the 
exercise type. When the therapeutic life style modification is used for the early hypertension patients, the combination of walking and intense exercise is the best option for lowering DBP.

\section{M-50 \\ Pulse and Hemodynamic Energy}

Ho Sung Son, Chi Bum Ahn, Jung Joo Lee, Kuk Hui Son, Jae Seung Jung, Hyun Koo Kim, Choon Hak Lim, Kyung Sun

Thoracic and Cardiovascular Surgery, Anesthesia and Pain Medicine, Korea Artificial Organ Center, Korea

University, Seoul, Korea

Background: Pressure gradient is not the driving force to move the fluid between two points but energy gradient is. And, pulse pressure is not a definite index for blood pulsatility but hemodynamic energy difference is. We have compared the physiologic effects of pulsatile vs. non-pulsatile blood flow in various cardiovascular situations by using the concepts of hemodynamic energy of energy equivalent pressure (EEP; $\mathrm{mmHg}$ ) and surplus hemodynamic energy (SHE; erg/cm3).

Methods: An pulsatile ECLS and a centrifugal pump were used to generate pulsatile and nonpulsatile blood flow. Experiments were performed under the condition of cardiopulmonary bypass.

Results: As for the kidney tissue perfusion, pulsatile blood flow showed a better effect than nonpulsatile flow ( $47.5 \pm 18.3$ vs. $83.4 \pm 28.5 \mathrm{ml} / \mathrm{min} / 100 \mathrm{~g} ; \mathrm{p}=0.026$ ). Coronary blood flow was higher with pulsatile blood flow than with nonpulsatile blood flow. As for the pump output, the parallel circuit configuration provides higher flow than the serial circuit configuration by doubling the pulse rate at a given pump-setting rate. (at 40 and 50 BPM pump-setting rates, $3.1 \pm 0.2 \& 3.7 \pm 0.2 \mathrm{~L} / \mathrm{min}$ vs. $2.2 \pm 0.1 \& 2.5 \pm 0.1 \mathrm{~L} / \mathrm{min}$, respectively, $\mathrm{p}=0.01$ ). Although a centrifugal pump alone was nonpulsatile, adding IABP to a centrifugal pump converted the blood flow to pulsatile and increased pulse pressure (from $9.1 \pm 1.3 \mathrm{~mm} \mathrm{Hg}$ to $54.9 \pm 6.1 \mathrm{mmHg}, \mathrm{p}=0.012$ ). It also increased the percent change from mean arterial pressure to EEP (from $0.2 \pm 0.3 \%$ to $23.3 \pm 6.1 \%, p=0.012$ ) and SHE (from $133.2 \pm 234.5$ to $20,219.8 \pm 5842.7 \mathrm{erg} / \mathrm{cm} 3, \mathrm{p}=0.012$ ). Combination of centrifugal pump and IABP showed same effects in coronary flow and hemodynamic energy compared to a pulsatile ECLS. Non pulsatile pump required $25 \%-28 \%$ higher pump flow than the pulsatile pump to maintain equivalent coronary blood flow in the fibrillating heart. the change in hemodynamic energy induced by dopamine, esmolol, and epinephrine may be expressed in terms of the EEP and SHE. In addition, there was a strong correlation between the EEP and flow. During ischemic-reperfusion, SHE was found to be a more sensitive hemodynamic energy parameter during ischemia-reperfusion injury than PP or EEP ( $p<0.05$ ). SHE and EEP was disappeared with $75 \%$ stenosis of vessel diameter.
Conclusion: The precise quantifications of pressure flow waveforms, EEP and SHE are valuable tools for evaluating pulsatility in the circulatory system. They are expected to be used as additional performance indexes of a blood pump.

\section{M-51 \\ In Comparison of Normotensive Subjects, Left Atrial Volume Index Independently Predicts Hypertensive Response to Exercise in Patients with Hypertension}

Ah-Young Ji, Jong-Chan Youn, Yongsung Suh, Jaewon Oh, Sungha Park, Sang-Hak Lee, Yangsoo Jang,

Seok-Min Kang

Yonsei college of medicine

Background: A hypertensive response to exercise (HRE) is associated with higher risk of future cardiovascular events. We investigated what factors are relevant to HRE in both hypertensive patients and normotensive control subjects.

Method: 95 hypertensive patients ( 44 men, $59 \pm 10$ years) and 60 normotensive control subjects ( 24 men, $54 \pm 8$ years) were consecutively enrolled. HRE was defined by maximum $\mathrm{SBP} \geq 210 \mathrm{mmHg}$ in males and $\geq 190 \mathrm{mmHg}$ in females. Clinical characteristics, echocardiographic and laboratory findings were assessed at the time of enrollment.

Results: HRE was more prevalentin hypertensive patients compared with normotensive control subjects (48.4\% vs. $21.7 \%, p=0.001)$. Peak oxygen uptake was lower in hypertensive patients ( $27.8 \pm 65 \mathrm{vs} .30 .5 \pm 6.0 \mathrm{~mL} / \mathrm{kg} / \mathrm{min}, \mathrm{p}=0.002)$, however there was no significant difference according to HRE. Age and body mass index were shown to be associated with HRE in normotensive control subjects. Multivariate analysis revealed that left atrial volume index (LAVI) was found to be an independent predictor of HRE in hypertensive patients $(\mathrm{p}=$ 0.003 ) but not in normotensive control subjects $(p=0.926)$ when controlled for age, gender, body mass index and renal function.

Conclusion: Higher LAVI, a surrogate marker of chronic diastolic dysfunction is independently related to HRE in hypertensive patients. 


\section{M-52}

\section{Factors Influencing the Time-course of Flow-Mediated Dilatation at Brachial Artery}

\author{
Lu Zhang, Ting-Yan Xu, Fang-Fei Wei, Yan Li
}

Center for Epidemiological Studies and Clinical Trials and Center for Vascular Evaluations, Ruijin Hospital, Shanghai Institute of Hypertension, Shanghai Jiao Tong University School of Medicine

Background: Flow-mediated dilatation (FMD) is an endothelium-dependent process in response to increased shear stress. It has been well accepted that the magnitude of FMD is an independent predictor for cardiovascular risk. However, attention paid to the time-course of FMD was limited. The aim of our current study was to explore which factors may influence the time-course of FMD.

Methods: From Dec 2011 to March 2012, 74 consecutive outpatients from our Hypertension Clinic were invited to participate in our study if they were not on antihypertensive medication for at least 2 weeks. Standardized questionnaires were administered to collect information on lifestyle, medications and disease history. FMD was measured with a dedicated ultrasonography system (UNEXEF18G, UNEX, Nagoya, Japan) coupled with a computer-assisted analysis software. The magnitude of FMD (\%FMD) was calculated as the percentage of maximum dilatation to the baseline diameter. The time to the maximum dilatation (Tmax) was log-transformed for analysis.

Results: The 74 participants included 43 (58.1\%) men and $51(68.9 \%)$ hypertensive patients. Age averaged 50 years. 19 subjects (all men) reported tobacco use and 26 alcohol consumption (23 men). The mean $\%$ FMD was $5.9 \pm 2.7 \%$. The median Tmax was 47 (interquartile range, 42 to 66) seconds. Women had higher Tmax than men (53 vs. 47 seconds, $\mathrm{P}=$ 0.12 ), but the difference did not reach statistical significance. In multivariate regression model, age ( 1.1 seconds per 10 year increase, $P=0.048$ ) and current alcohol intake $(P=0.002)$ were significantly associated with Tmax, after accounting for sex, body mass index, current smoking, blood glucose, total cholesterol, mean blood pressure, baseline diameter and shear stress. Drinkers had a significantly greater Tmax than non-drinkers ( 61 vs. 46 seconds, $P=0.003$ ), and this trend ( 60 vs. 47 seconds, $\mathrm{P}=0.08$ ) remained true when the multivariateadjusted analysis was limited in men.

Conclusions: Older age and alcohol consumption delayed the maximum dilation at brachial artery in response to the stimulus. It highlighted the necessity of continuous monitoring of the dilatation during the measurement of FMD. Future studies are warranted to explore if the parameters related to the time-course of FMD would have any value in risk stratification.

\section{M-53}

Age-Associated Increase in Arterial Stiffness Measured by Cardio-Ankle Vascular Index Without Blood Pressure Change in Healthy Adults

Su-Yeon Choi ${ }^{1}$, Byung-Hee Oh${ }^{2}$, Jeong Bae Park', Dong-Ju Choi ${ }^{4}$, Moo-Yong Rhee ${ }^{5}$, Sungha Park ${ }^{6}$

${ }^{1}$ Department of internal medicine, Healthcare system gangnam center, Seoul national university hospital, Seoul, Korea, ${ }^{2}$ Department of internal medicine, Seoul national university hospital, Seoul, Korea, ${ }^{3}$ Department of internal medicine, Kwandong university college of medicine, Cheil general hospital, Seoul, Korea, ${ }^{4}$ Department of internal medicine, Seoul national university bundang hospital, Seongnam-si, Gyeonggido, Korea, ${ }^{5}$ Department of internal medicine, Dongguk university ilsan hospital, Goyang-si, Gyeonggi-do, Korea, ${ }^{6}$ Department of internal medicine, Yonsei university college of medicine, Cardiovascular hospital, Seoul, Korea

Background : The cardio-ankle vascular index (CAVI) reflects arterial stiffness from aorta to the ankle, less dependent of blood pressure (BP). We investigated the agestratified CAVI in healthy people to evaluate the effect of age on arterial stiffness.

Methods: CAVI and peripheral BP were determined in healthy and normotensive Koreans aged 20 to 79 years. Subjects had no history of cardiovascular disease and were free from taking medications for hypertension, diabetes mellitus or dyslipidemia ( $\mathrm{N}=1,380 ; 44.1 \%$ in males).

Results: Mean systolic blood pressure (SBP), diastolic blood pressure (DBP) and pulse pressure (PP) were 117, 75, and $42 \mathrm{mmHg}$ respectively. CAVI increased linearly with age. CAVI was determined using the following equation; CAVI $=5.0$ $+0.048 \mathrm{x}$ age (year) in males $(\mathrm{r} 2=0.395, \mathrm{p}<0.001)$, CAVI $=4.8$ $+0.045 \mathrm{x}$ age (year) in females ( $\mathrm{r} 2=0.450, \mathrm{p}<0.001$ ). However, SBP, DBP and PP did not change progressively with age. Age emerged as the major determinants of CAVI in stepwise multiple regression ( $\mathrm{r} 2$ change $=43.1 \%$ ).

Conclusion: CAVI score increased with age in healthy and normotensive individuals, whereas SBP, DBP and PP did not. Age was the dominant risk factor for the progression of arterial stiffness. These data suggest that CAVI might be a sensitive marker of arterial aging process above and beyond conventional upper arm BP. 


\section{M-54 \\ The Correlation of Carotid Artery Stiffness with Heart Function in Hypertensive Patients}

Hye-Sun Seo, Nae-hee Lee, Jon Suh, Jae Huk Choi

Soonchunhyang University Hospital, Bucheon, Korea,

Republic of Korea

Background: The strength of each heart beat and the stiffness of large arteries contribute to blood pressure. When the large arteries are stiff and their resistance greater, the afterload increases and this may change the functioning of the heart. However, the relation between common carotid artery stiffness and heart function in hypertensive patients has not been clarified.

Methods: Two hundred and twenty hypertensive patients underwent transthoracic and carotid echocardiography. Measurements of local arterial stiffness were taken at the right common carotid artery level and stiffness parameter $(ß)$, pressure-strain elasticity modulus (Ep) and intimamedia thickness (IMT) were calculated. Brachial cuff BP was measured just before starting the carotid study. The patients with any cardiovascular disease, diabetes mellitus (DM), stroke, transient ischemic attack (TIA), or carotid stenosis were excluded.

Results: Carotid artery stiffness parameter ( $($ ) was correlated with age and left ventricular (LV) mass index ( $\mathrm{p}<0.005)$. Even though $ß$ was not correlated with LV systolic function, it was inversely well correlated with diastolic function. When the artery was stiffer, early mitral annular velocity ( $\left.E^{\prime}\right)$ decreased $(\mathrm{p}<0.001)$ and the index of left atrial (LA) pressure (early diastolic mitral inflow E velocity / E') increased ( $p=0.001)$. In logistic regression, diastolic dysfunction was affected by age (beta $-0.385, p=0.001$ ), LA volume index (beta $0.175, p=$ 0.013 ) and $\beta$ (beta $-0.273, p=0.019$ ).

Conclusion: In hypertensive patients, changes in carotid artery stiffness can affect the diastolic function, independent of age and LA volume index. Therefore, measurements and control of carotid stiffness can play an important role in the prevention of diastolic heart failure.

\section{M-55 \\ Estimation of Central Aortic Systolic Pressure by Applying an $\mathrm{N}$-point Moving Average on a Cuff-Derived Brachial Pressure Waveform}

Hao-Min Cheng 1,2,3, Yuan-Ta Shih ${ }^{5}$, Shih-Hsien Sung ${ }^{1,3,4}$, Chen-Huan Chen 1,2,3,4

${ }^{1}$ Department of Medicine, and ${ }^{2}$ Department of Medical Research and Education, Taipei Veterans General Hospital, ${ }^{3}$ Department of Medicine, ${ }^{4}$ Department of Public Health, Faculty of Medicine, National Yang-Ming University, Taipei, Taiwan, R.O.C.; ${ }^{5}$ Molecular imaging center, National Taiwan University

Background: An N-point moving average (NPMA) is a mathematical low-pass filter that can smooth a peaked noninvasively acquired tonometric radial pressure waveform to provide an estimate of central aortic systolic blood pressure (SBP-C), when using a common denominator of $n / 4$ (where $\mathrm{n}=$ tonometer sampling frequency). The aim of the present study was to investigate if the NPMA method can be applied to a brachial pulse volume plethysmography (PVP) waveform.

Methods: 1) Development study: Simultaneously recorded invasive high-fidelity brachial and central aortic pressure waveforms from 40 subjects were analyzed to identify the best common denominator; 2) Validation study: Simultaneously recorded invasive high-fidelity central aortic pressure waveforms and noninvasive brachial PVP waveforms from 140 subjects were analyzed. PVP waveforms were calibrated to the cuff systolic and diastolic blood pressures.

Results: In the development study, a denominator of $n / 6$ was identified. The mean difference between the estimated (with the invasive brachial pressure waveforms by the NPMA, $\mathrm{n} / 6$ ) and measured SBP-C was $0.1 \pm 3.5 \mathrm{mmHg}$. In the validation study, the mean difference between the estimated (with the non-invasively calibrated PVP waveforms by the NPMA, n/6) and the measured SBP-C was $-0.7 \pm 7.6 \mathrm{mmHg}$. For comparison, the mean difference was $-2.1 \pm 7.6 \mathrm{mmHg}$, when SBP-C was estimated by a generalized transfer function method.

Conclusions: This study demonstrates that a NPMA with a common denominator of $n / 6$ can be applied to the PVP waveforms to accurately estimate SBP-C. Brachial pressure waveforms require a less strong low-pass filter than radial pressure waveforms to define SBP-C. A NPMA with a denominator of $\mathrm{n} / 6$ can easily be incorporated into an oscillometric blood pressure monitor to measure SBP-C. 


\section{M-56 \\ Effects of Nicorandil on Aorta Stiffness in Patients with Acute Coronary Syndrome}

Jeong-Sook Seo, Young Ah Park, Jae Hyun Park, Uk Kang, Yeo Jung Song, Kyu-Nam Choi, Han Young Jin, Jae-Sik Jang, Tae-Hyun Yang, Dae-Kyeong Kim, Dong-Soo Kim

Division of Cardiology, Department of Internal Medicine, Busan Paik Hospital, Inje University, Busan, Republic of Korea

Background: The different classes of vasodilators have different mechanisms affecting systemic vessels. Therefore, they produce different changes in aortic stiffness. The purpose of this study was to assess the vasodilator effects of nicorandil on aortic stiffness and to compare these effects with nitrate in patients with acute coronary syndrome (ACS) after percutaneous transcatheter angioplasty (PTCA).

Methods: The study was designed as a single-blind, randomized prospective study. Nineteen patients (18 Men, mean age $62.5 \pm 12.2$ years) with ACS underwent PTCA were divided into 2 groups. The effects after 8 weeks of treatment with nicorandil (3x $5 \mathrm{mg} /$ day) and isosorbide mononitrate (ISMN, 1 x 60mg/day) on aortic stiffness were evaluated. Aortic stiffness was measured with pulse wave velocity (PWV) and augmentation index (AIx) using VP-2000.

Results: Five patients discontinued the study in treatment period due to headache, four with ISMN and one with nicorandil. At baseline, age, systolic blood pressure (BP), diastolic BP, heart rate, branchial-ankle PWV and AIx were not different between two groups. After 8 weeks, systolic BP, diastolic $\mathrm{BP}$, heart rate, $\mathrm{PWV}$ and $\mathrm{AIx}$ were not change in each group. The differences of hemodynamic parameters, PWV and AIx were not different in both groups.

Conclusion: Aortic stiffness was not improved both with nicorandil and ISMN. No differences between ISMN and nicorandil were found for mid-term effects on aortic stiffness.

\section{M-57 \\ Microvascular Findings in Patients with Raynaud's Disease: Assessment by Nailfold Capillaroscopy and Flow Mediated Dilatation}

Kyoung-Im Cho ${ }^{7}$, Jung-Ho Heo ${ }^{7}$, Hyun-Soo Kim ${ }^{7}$, Tae-Joon Cha ${ }^{1}$, Jae -Woo Lee ${ }^{1}$, Ji-Hyun Lee ${ }^{2}$

${ }^{1}$ Department of Intenal Medicine, Kosin University School of Medicine, Busan, Korea, ${ }^{2}$ Department of Internal Medicine, Maryknoll Medical Center, Busan, Korea

Background: Nailfold capillaroscopy (NC) is a method to analyze microvascular abnormalities in autoimmune rheumatic diseases. However, the pathophysiological link between microvascular derangement seen in $\mathrm{NC}$ and endothelial function has yet to be elucidated. We investigated the association between endothelial function, assessed by brachial artery flow-mediated dilatation (FMD), and NC patterns in patients with Raynaud's phenomenon (RP).

Methods: Postmenopausal women ( $\mathrm{n}=37$ ) with secondary RP and women with hand numbness (controls, $\mathrm{n}=$ 23) were evaluated with NC using a digital microscope at 400x and $100 \times$ magnifications. Microvascular alterations were classified into early, active, and late patterns. Endothelial function was examined by brachial FMD (endothelium dependent) and response to $40 \mu \mathrm{g}$ of sublingual nitroglycerine (NTG-induced dilatation, endothelium independent).

Results: There was significant capillary loop dilatation (apical width; $14.1 \pm 5.6$ vs. $10.4 \pm 1.7 \mu \mathrm{m}, \mathrm{p}=0.001$ and total width; $40.6 \pm 15.1$ vs. $31.6 \pm 4.6 \mu \mathrm{m}, \mathrm{p}=0.002$ ) and lengthening ( $316.0 \pm 78.5$ vs. $270.4 \pm 34.7 \mu \mathrm{m}, \mathrm{p}=0.004$ ) in secondary RP compared to controls. Additionally, giant capillaries, loss of capillaries, hemorrhage, and background pallor were much more prevalent in secondary RP as compared to controls ( $\mathrm{p}$ $<0.05$ for all variables). Although there were no significant differences in NTG-induced dilatation between secondary RP and controls (16.1 $\pm 5.9 \%$ vs. $19.6 \pm 9.0 \%, p=0.091)$, significant decreases in the FMD value $(6.1 \pm 3.5 \%$ vs. $9.0 \pm 2.2 \%, \mathrm{p}<0.001)$ were noted. Both FMD and NTG-induced dilatation showed a significant inverse association with severity of NC findings $(\mathrm{r}=-0.355, \mathrm{p}=0.005$ and $\mathrm{r}=-0.285, \mathrm{p}=0.28)$.

Table 1. (for Abstract M-56)

\begin{tabular}{llllll}
\hline & \multicolumn{2}{l}{ ISMN group (n=7) } & & \multicolumn{2}{l}{ Nicorandil group (n=7) } \\
\cline { 2 - 3 } \cline { 5 - 6 } & Baseline & 8 weeks & & Baseline & 8 weeks \\
\hline Systolic BP, mmHg & $133.3 \pm 13.9$ & $131.0 \pm 19.2$ & & $129.4 \pm 17.4$ & $137.6 \pm 23.4$ \\
Diastolic BP, mmHg & $79.1 \pm 10.1$ & $71.4 \pm 13.2$ & & $78.43 \pm 9.2$ & $78.9 \pm 12.4$ \\
Heart rate, bpm & $75.6 \pm 24.0$ & $72.6 \pm 17.5$ & & $73.0 \pm 9$ & $66.6 \pm 12.6$ \\
baPWV & $1683.7 \pm 336.1$ & $1455.4 \pm 216.6$ & & $1798.3 \pm 471.6$ & $1577.7 \pm 362.7$ \\
faPWV & $1066.4 \pm 169.2$ & $977.9 \pm 170.5$ & & $1105.3 \pm 150.5$ & $1004.2 \pm 106.1$ \\
hfPWV & $1285.3 \pm 488.6$ & $1061.3 \pm 235.0$ & & $1106.3 \pm 310.1$ & $1139.8 \pm 256.4$ \\
hcPWV & $1077.4 \pm 367.7$ & $875.3 \pm 245.5^{*}$ & & $895.2 \pm 174.2$ & $793.5 \pm 148.9$ \\
AIx, carotid, \% & $16.3 \pm 11.9$ & $5 \pm 23.7$ & & $12.0 \pm 12.1$ & $21.50 \pm 13.9$ \\
AIx, brachial, \% & $12.6 \pm 16.8$ & $9 \pm 13.1$ & & $19.1 \pm 14.9$ & $12.9 \pm 17.4$
\end{tabular}


Conclusions: Significantly impaired endothelial function was found in secondary RP, and capillary enlargement was significantly associated with endothelial dysfunction. This suggests that the recognition of abnormal capillaroscopic patterns in secondary RP might be prognostic for early detection of microvascular heart complications.

\section{M-58 \\ Clinical Validation of Korean Version of Peripheral Artery Questionnaire in Peripheral Artery Disease: Its Relationship with Ankle- Brachial Index - KPEARL Study: A Cross- sectional, Multi-center, Observational Study \\ Kyoung-Im Cho ${ }^{1}$, Bo-Hyun Kim², Jae-Sik Jang ${ }^{3}$, Yong Hyun Park ${ }^{4}$, Hyoung-Gon Je ${ }^{5}$, Mi-Seong Shin', Ji-Hyun Lee ${ }^{7}$ \\ ${ }^{1}$ Department of Intenal Medicine, Kosin University School of Medicine, Busan, Korea, ${ }^{2}$ Department of Internal Medicine, Pusan National University Hospital, Busan, Korea, ${ }^{3}$ Department of Internal Medicine, Busan Paik Hospital, University of Inje College of Medicine, Busan, Korea, ${ }^{4}$ Department of Internal Medicine and ${ }^{5}$ Department of Cardiovascular Surgery, Pusan National University Yangsan Hospital, Yangsan, Korea, ${ }^{6}$ Department of Intenal Medicine, Gil Hospital, Gacheon University School of Medicine, Incheon, Korea, ${ }^{7}$ Department of Internal Medicine, Maryknoll Medical Center, Busan, Korea}

Background: Peripheral Artery Questionnaire (PAQ) was developed to monitor health status of patients with peripheral arterial disease (PAD) patients. However, to date, its correlation with traditional screening test, ankle brachial index (ABI) is unknown. The aim of this study was to establish the further clinical validity of the PAQ by examining its association with $\mathrm{ABI}$ in patients with exertional leg symptoms.

Methods: The Korean PAQ (KPAQ) was completed by 567 patients with suspicious intermittent claudication in this multicenter, cross-sectional, non-interventional study. Demographics, medical history, physical examination findings, and ABI of the patients were analyzed. The KPAQ was analyzed according to the six domains and the summary score. The number of patients with low ABI (0.90) was analyzed and assessed the relationship between summary score of PAQ and ABI.

Results: The frequency of low ABI was $43 \%$ in the whole study population and hypertension and dyslipidemia were present in $53 \%$ and $49.7 \%$ of patients, respectively. Summary score of PAQ was significantly lower in patients with low ABI compared to those normal ABI (37.6 \pm 19.0 vs. $70.1 \pm 22.7, \mathrm{p}<$ $0.001)$. There was significant association between summary score of PAQ and ABI ( $r=0.558, \mathrm{p}<0.001)$, and the cut off value of summary score for predicting low ABI was 50.3 (AUC $=0.857)$. Older age, greater number of total risk factors, and presence of PAD-related physical findings were associated with increased likelihood of lower summary score of PAQ $(\mathrm{p}<0.001)$.
Conclusion: Our results indicate that summary score of PAQ were associated with increased likelihood of presence of PAD in patients with exertional leg symptoms, and low summary score (50.3) might have a good predictive power for low ABI (0.90).

\section{M-59 \\ Quantifying Improvement in Health Status Measure After Peripheral Revascularization Treatment Using Peripheral Artery Questionnaire (PAQ) and Ankle-Brachial Index (ABI); A Cross-Sectional, Single-Center, Observational Study

\author{
Kyoung-Im Cho ${ }^{1}$, Bo-Hyun Kim², Jae-Sik Jang ${ }^{3}$, \\ Yong Hyun Park ${ }^{4}$, Hyoung-Gon Je \\ ${ }^{1}$ Department of Intenal Medicine, Kosin University \\ School of Medicine, Busan, Korea, ${ }^{2}$ Department of \\ Internal Medicine, Pusan National University Hospital, \\ Busan, Korea, ${ }^{3}$ Department of Internal Medicine, Busan \\ Paik Hospital, University of Inje College of Medicine, \\ Busan, Korea, ${ }^{4}$ Department of Internal Medicine and \\ ${ }^{5}$ Department of Cardiovascular Surgery, Pusan National \\ University Yangsan Hospital, Yangsan, Korea
}

Background: The current study aims to quantify improvement of health status after peripheral revascularization using peripheral artery questionnaire (PAQ) and anklebrachial index (ABI) and to evaluate correlation between two methods.

Methods: Between January 2009 and August 2012, 218 consecutive patients underwent peripheral revascularization treatment for symptomatic peripheral arterial disease. In this cross-sectional, descriptive-analytic research, 149 patients fulfilled pre and post-revascularization PAQ were enrolled. The PAQ scores range from 0 to 100; higher scores represent fewer symptoms and better health status. Of these, pre and post ABI were evaluated in 81 patients. Characteristics of studied subjects including demographics, familial history, past medical history and atherosclerotic risk factors such as diabetes mellitus, hypertension, hyperlipidemia and smoking were obtained using a standard questionnaire.

Results: Mean age was $70 \pm 10$ years, $83.9 \%$ were men. Hypertension, diabetes, dyslipidemia and smoking were present in $63.8 \%, 38.9 \%, 61.7 \%$ and $46.3 \%$ of patients, respectively. Mean PAQ summary scores improved significantly after revascularization from $32.1 \pm 11.3$ to $81.4 \pm 16.4$ at 4 weeks (P $<0.001$ ) and the improvement of PAQ summary scores was comparable according to revascularization method (intervention; $50.0 \pm 14.0$ vs. surgery $47.7 \pm 19.0, p>0.1$ ). The change after revascularization was biggest in symptoms category $(60.5 \pm 25.2)$ and smallest in treatment satisfaction (32.6 \pm 17.5$)$. There was significant change on mean ABI of affected limb $(0.31 \pm 0.23, p<0.01)$. The changes of score in physical function $(\mathrm{r}=0.326, \mathrm{p}=0.003)$ and quality of life $(\mathrm{r}=0.220, \mathrm{p}=0.048)$ domain showed significant correlation to changes of $\mathrm{ABI}$. 
Conclusion: In this study, PAQ scores and ABI well document numerical improvements after peripheral revascularization regardless of revascularization methods. The improvement of PAQ scores is associated with changes of ABI.

\section{M-60 \\ Uncommon Cause of Acute Deep Vein Thrombosis - The Case Report of May- Thurner Syndrome (May-Thurner Syndrome: An Unexpected Finding in a Patient with Swelling Left Leg)}

Nongnooch Poowanawittayakom, Haq Wajid, Prangthip Chareonpong, Ravi Narra, Samee Mohammed

Department of Medicine, Advocate Illinois Masonic Medical Center, Chicago, IL (60657)

May-Thurner syndrome is an unusual condition of deep venous thrombosis (DVT) in the illiofemoral vein that is caused by anatomical malformation of the right common iliac artery overlying on the left common illiac vein. It is still questionable whether this vascular abnormality will be recognized as a pathological or normal variant because this condition is found approximately $2-5 \%$ in lower extremity venous disorders and occurs only after precipitating incidents such as minor trauma or orthopedic surgery.

A 46-year-old Caucasian woman was presented at ED after fall at home with left leg swelling for 2 days. The patient denied recent travel and oral contraceptive use. Physical examination showed severe swelling, warm, bluish and redness along the left calf.. The Duplex ultrasonography was done to exclude DVT and revealed extensive DVT of left iliac vein, common femoral vein, superficial femoral vein, and popliteal vein. Laboratory results for hypercoagulable states, including factor $\mathrm{V}$ Leiden, antithrombin III, protein C deficiency, protein $\mathrm{S}$ deficiency, and D-dimer were negative. Therefore, CT abdomen was done to rule out possible abdominal cancer or obstruction. Unexpectedly, the finding showed a rare vascular abnormality, which was the absence of left common iliac vein with the dilation of distal left iliac vein. The patient was treated with catheter-directed thrombolysis, stent replacement at left iliac vein, and temporary IVC filter and was sent home with warfarin for at least 6 months and lifelong aspirin $81 \mathrm{mg}$. daily.

May-Thurner syndrome is a rare disease of the vascular malformation that requires specific treatments, both anticoagulation therapy and stent placement, to prevent the recurrence of the symptoms. It is important for the physicians to include May-Thurner syndrome in the differential diagnosis when taking care of patients presenting with swelling left leg.

\section{M-61 \\ The Impact of Metabolic Syndrome on Coronary Artery Spasm as Assessed with Intra Coronary Acetylcholine Provocation Test}

\author{
Se Yeon Choi, Seung-Woon Rha, Byoung Geol Choi, \\ Cheol Ung Choi, Chang Gyu Park, Hong Seog Seo, \\ Dong Joo Oh \\ Cardiovascular Center, Korea University Guro Hospital, \\ Seoul, Korea
}

Background: Metabolic Syndrome (MS) has been known to be an important cardiovascular risk factor. We investigated whether the presence of MS can impact on coronary artery spasm (CAS) as assessed with intracoronary acetylcholine (Ach) provocation test.

Methods: A total 3490 consecutive patients (pts) who underwent coronary angiography with Ach provocation test were enrolled. National Cholesterol Education Program Adult Treatment Panel III (NCEP-ATP III) was used for the definition of the MS. The pts were divided into two groups; MS group ( $n=680,19.5 \%)$ and non-MS group $(n=2810,80.5 \%)$ groups. Ach provocation test was performed by incremental dosage $(20,50,100 \mathrm{ug})$ into left coronary artery. Significant CAS was defined more than $>70 \%$ luminal narrowing visually with chest pain with/without ST segment changes.

Results: Baseline characteristics were balanced between the two groups except the MS group had more elderly (53.92 \pm 12.7 vs. $59 \pm 10.6, \mathrm{p}<0.001)$, hypertension $(83.1 \%$ vs. $36.6 \%, \mathrm{p}<0.001$ ), diabetes ( $35.6 \%$ vs. $5.7 \%, \mathrm{p}<0.000$ ), dyslipidemia $(24.1 \%$ vs. $14.1 \%, \mathrm{p}<0.001)$, and cerebrovascular accident (3.5\% vs. $2.1 \%, \mathrm{p}=0.024)$, whereas the male gender ( $40.7 \%$ vs. $47.5, \mathrm{p}=0.001)$, peripheral vascular disease $(1.3 \%$ vs. $2.7 \%, \mathrm{p}=0.040)$, current smoking $(17.4 \%$ vs. $21.1 \%, \mathrm{p}=$ 0.028 ) and alcohol (26.3\% vs. $32.6 \%, p=0.001$ ) were lower in the MS group. Angiography characteristics during Ach provocation test, the MS group showed higher incidence of QCA measured severe CAS, mean \% narrowing and smaller mean diameter than non-MS group (Table).

Conclusion: According to our study, the MS pts had more coronary risk factors and worse angiographic characteristics during Ach provocation test, suggesting more careful medical therapy and follow up would be required.

Table 1. Angiographic parameters of the study groups (for Abstract M-61)

\begin{tabular}{llll}
\hline Variables, $\mathrm{n}(\%)$ & $\begin{array}{l}\text { Non-metabolic } \\
\text { syndrome } \\
(\mathrm{n}=2810)\end{array}$ & $\begin{array}{l}\text { Metabolic } \\
\text { syndrome } \\
(\mathrm{n}=680)\end{array}$ & P-value \\
\hline Ach induce spasm (visual > 70\%) & $1614(57.4)$ & $407(59.9)$ & 0.252 \\
Myocardial bridge & $535(19)$ & $115(16.9)$ & 0.201 \\
Diffuse lesion (> 30mm) & $1357(84.1)$ & $339(83.3)$ & 0.700 \\
Multivessel spasm & $537(33.3)$ & $126(31)$ & 0.374 \\
Baseline spasm (> 30\%) & $606(21.6)$ & $135(19.9)$ & 0.327 \\
QCA assessed spasm & & & \\
$\quad$ QCA, <50\% & $80(5)$ & $18(4.4)$ & 0.654 \\
$\quad$ QCA, 50-70\% & $650(40.3)$ & $130(31.9)$ & 0.002 \\
$\quad$ QCA, > 70\% & $882(54.6)$ & $258(63.4)$ & 0.001 \\
Narrowing diameter, mm & $0.71 \pm 0.36$ & $0.65 \pm 0.33$ & 0.001 \\
Mean \% narrowing, \% & $70.47 \pm 13.05$ & $72.02 \pm 12.35$ & 0.027 \\
\hline
\end{tabular}




\section{M-62}

Relationship Between Resolution of STSegment Elevation and Microvascular Dysfunction in Patients Treated with Primary Percutaneous Coronary Intervention (PPCI); Index of Microvasculatory Resistance (IMR) Study

Byung gyu Kim, Hye Young Lee, Byung Ok Kim, Jeong Hoon Kim, Young-Sub Byun, Choong-Won Goh

Inje University Sanggye-Paik Hospital, Seoul, Republic of Korea

Background: and objectives The usefulness of ST-segment elevation resolution (STR) for predicting epicardial reperfusion is well established. However, it is not known how the ST-segment changes are related with microvascular obstruction. The aim of this study was to evaluate the relationship between the degree of ST segment resolution (STR) and the extent of microcirculatory dysfunction, which was assessed by TIMI myocardial perfusion (TMP) grade and Index of microvasculatory resistance (IMR).

Methods: A total of 55 consecutive patients with ST-segment elevation myocardial infarction (STEMI), who underwent successful reperfusion therapy (PPCI), were evaluated. Serial 12-lead electrocardiography (ECG) was performed at baseline and at 90 minutes after reperfusion therapy. Microvascular dysfunction was evaluated according to TIMI myocardial perfusion (TMP) grade and index of microvasculatory resistance (IMR), which was assessed immediately after reperfusion using thermodilution curves obtained during maximal hyperemia by intracoronary adenosine injection with a pressure/temperature sensor-tipped guide.

Results: Mean age of patients was 58 years. History of hypertension and diabetes under treatment was $60 \%$ and $24 \%$, respectively. Culprit lesions of IRA include 29 left anterior descending artery (52.7\%), 3 left circumflex artery (5.5\%), 22 right coronary artery (40\%), and 1 ramus intermedius (1.8\%). While the magnitude of STR was positively correlated with LVEF ( $r=0.321, p=0.03$ ), it was correlated neither with TMP $(\mathrm{r}=0.097, \mathrm{p}=0.484)$ nor with IMR $(\mathrm{r}=-0.034, \mathrm{p}=0.834)$. There was significant strong positive correlation between IMR and TMP grade ( $\mathrm{r}=0.421, \mathrm{p}=0.007)$.

Conclusions: STR reflects the left ventricular dysfunction (EF) after reperfusion rather than the extent of microcirculatory dysfunction (IMR and TMP).

\section{M-63 \\ Relationship Between Arterial Stiffness and Variability of Home Blood Pressure Measurements}

Jin-Sun Park', Joon-Han Shin ${ }^{7}$, Jeong-Bae Park ${ }^{2}$, Dong-Ju Choi ${ }^{3}$, Ho-Joong Youn ${ }^{4}$, Chang-Gyu Park ${ }^{5}$ Jun Kwan ${ }^{6}$, Young-Keun Ahn', Dong-Woon Kim ${ }^{8}$, Se-Joong Rim ${ }^{9}$, Seung-Woo Park ${ }^{10}$, Jidong Sung ${ }^{10}$, Jang-Ho Bae ${ }^{11}$ on behalf of the Korean Hypertension Research Network

${ }^{1}$ Ajou University School of Medicine, ${ }^{2}$ Cheil General Hospital, Kwandong University College of Medicine, ${ }^{3}$ Seoul National University Bundang Hospital, ${ }^{4}$ The Catholic University of Korea, ${ }^{5}$ Korea University Guro Hospital, ${ }^{6}$ Inha University College of Medicine, ${ }^{7}$ The Heart Center of Chonnam National University Hospital, ${ }^{8}$ Cardiovascular Center of Chungbuk National University Hospital, ${ }^{9}$ Yonsei University College Medicine, ${ }^{10}$ Samsung Medical Center, Sungkyunkwan University School of Medicine, ${ }^{11}$ Heart Center, College of Medicine, Konyang University

Background: Variability of blood pressure (BP) is known as a prognostic value for the subsequent target organ damage in hypertensive patients. Arterial stiffness is a risk factor for cardiovascular morbidity and mortality. The relationship between the arterial stiffness and the BP variability has been controversial. The objective of the present study was to investigate the relationship between arterial stiffness and home BP variability in patients with pre-hypertension (pre-HTN) and hypertension (HTN).

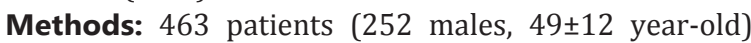
were enrolled. Using radial applanation tonometry, by pulse wave analysis (PWA) was performed for evaluation of systemic arterial stiffness. All patients underwent both home BP measurements and PWA. Patients were asked to measure their seated BP once every morning within 1 hour of waking, and once every evening 1 hour before going to bed, after more than 2 minutes of rest each time. Home BP variability was calculated as the standard deviation (SD) of 7 HBP measurements. Among these patients, 329 patients underwent 24-hour ambulatory BP monitoring (ABPM) for evaluating the additional effects of circadian variability. Multiple linear regression analysis was performed to estimate and test the independent effects of home BP variability on the arterial stiffness.

Results: Mutivariate analysis showed that both systolic and diastolic morning BP variabilities were correlated with arterial stiffness expressed as augmentation pressure (AP, $\beta=1.538, p=0.01$ and $\beta=1.374, p=0.035)$. The SDs of systolic and diastolic of evening were also associated with AP ( $\beta=$ $1.565, p=0.01$ and $\beta=1.397, p=0.036)$. The SDs of morning and evening systolic BP were associated with augmentation index (AI, $\beta=2.37, \mathrm{p}=0.02$ and $\beta=2.402, \mathrm{p}=0.001$ ) and heart rate $(75 \mathrm{bpm})$ adjusted $\mathrm{AI}(\beta=2.099, \mathrm{p}=0.01$ and $\beta=$ $2.115, p=0.001)$. No statistical difference between circadian $\mathrm{BP}$ variability and arterial stiffness was demonstrated. 
Conclusion: In present study, the variability of systolic BP was closely related with arterial stiffness in patients with HTN and pre-HTN. The home BP variability might be more significant indicator of arterial stiffness than circadian BP variability.

\section{M-64 \\ Half Marathon Was Better Than Marathon in Post-Exercise Hypotension}

Jae Hyoung Park, Su Jin Jung, Mi Na Lee, Hyung Joon Joo, Chul-Min Ahn, Soon Jun Hong, Do-Sun Lim

Korea University Anam Hospital, Cardiology

Background: Central blood pressure is independent predictors of cardiovascular risk. We investigated the acute effects of marathon and half marathon on central and peripheral blood pressures.

Methods: We studied 17 trained male amateur marathoners (mean age; 52.3 \pm 8.9 years). We measured augmentation index (AIx), flow mediated dilation (FMD), brachial ankle pulse wave velocity (ba-PWV), central and brachial blood pressure before and immediate after the Seoul international marathon (18th March 2012). And, 1 month later, we measured them again after half marathon. AIx, central and brachial blood pressure were measured using HEM9000AI (Omron Healthcare Co., Ltd., Kyoto, Japan). Repeated measured ANOVA and post-hoc Bonferroni correction were used to compare the difference between marathon and half marathon for AIx and blood pressure.

Results: There was no significant change of brachial systolicand diastolic blood pressure before andafter marathon. ( $p=0.369,0.903$, respectively) However, there was a significant reduction of brachial systolic blood pressure after half marathon (127.9 \pm 17.1 to $115.1 \pm 10.3 \mathrm{mmHg}$; $=0.004$ ). Both marathon and half marathon caused a significant reduction of central blood pressure ( $\mathrm{p}=0.019, \mathrm{p}<0.001$, respectively). However, there is no significant difference between marathon and half marathon in central blood pressure. There was no significant change of FMD and ba-PWV in marathon and half marathon.

Conclusion:Thepresentstudy found a reduction incentral systolic blood pressure in marathon and half marathon. There
Table 1. Changes of blood pressures in marathon and half marathon (for Abstract M-64)

\begin{tabular}{|c|c|c|c|}
\hline $\begin{array}{l}\text { Variables } \\
\text { (mmHg) }\end{array}$ & $\begin{array}{l}\text { Baseline } \\
(\text { mean } \pm S D)\end{array}$ & $\begin{array}{l}\text { Marathon } \\
\text { (mean } \pm \text { SD) }\end{array}$ & $\begin{array}{l}\text { Half marathon } \\
\text { (mean } \pm \text { SD) }\end{array}$ \\
\hline Central SBP & $130.2 \pm 19.4$ & $116.6 \pm 17.4^{*}$ & $109.3 \pm 9.3^{* * *}$ \\
\hline Brachial SBP & $127.9 \pm 17.1$ & $121.5 \pm 14.5$ & $115.1 \pm 10.3^{* *}$ \\
\hline Brachial DBP & $77.5 \pm 12.4$ & $74.9 \pm 9.6$ & $73.9 \pm 7.8$ \\
\hline
\end{tabular}

${ }^{*} \mathrm{p}<0.05$ versus baseline, ${ }^{* *} \mathrm{p}<0.01$ versus baseline, ${ }^{* * *} \mathrm{p}<0.001$ versus baseline.

was significant reduction in brachial systolic blood pressure in half marathon, but, not in marathon. Both marathon and half marathon had acute beneficial effects on cardiovascular system contrary to our concerns. However, half marathon was better than marathon in post-exercise hypotension.

\section{M-65 \\ Endothelial Progenitor Cells (EPCS) Increased Immediately After Full Course Marathon Race}

Jae Hyoung Park, Su Jin Jung, Ji-Hyun Choi, Hyung Joon Joo, Chul-Min Ahn, Soon Jun Hong,

Do-Sun Lim

Korea University Anam Hospital, Cardiology

Background: Exercise is thought to stimulate the release of hematopoietic and endothelial progenitor cells (EPCs) from the bone marrow. The aim of this study was to investigate the influence of a marathon race on the amount of circulating progenitor cells immediately after the race.

Methods: We studied 30 trained amateur marathoners (male $\mathrm{n}=28$, mean age $=51.6 \pm 8.3$ years). We measured flow mediated dilation (FMD) before and immediate after the Seoul international marathon (18th March 2012). Circulating numbers of $\mathrm{CD}_{3} 4^{+} / \mathrm{KDR}^{+}, \mathrm{CD} 34^{+} / \mathrm{CD} 117^{+}$and $\mathrm{CD} 34^{+} / \mathrm{CD} 133^{+}$ EPCs were quantified by flow cytometry at baseline and again after race.

Results: A marathon race led to a significant increase in white blood cell count $(5613 \pm 1804$ to $13148 \pm 3584$ cells/

Table 1. Changes of FMD and EPCs after marathon (for Abstract M-65)

\begin{tabular}{|c|c|c|c|}
\hline Variables & Pre-race $($ mean \pm SD) & Post-race (mean \pm SD) & $P$ value \\
\hline FMD (\%) & $16.6 \pm 12.0$ & $9.7 \pm 7.2$ & $\mathrm{P}=0.024$ \\
\hline \multirow[t]{2}{*}{ WBC (cells/uL) } & $5613.3 \pm 1804$ & $13148 \pm 3484$ & $\mathrm{P}<0.001$ \\
\hline & Pre-race (mean \pm SE) & Post-race (mean \pm SE) & \\
\hline \multicolumn{4}{|l|}{ Double staining } \\
\hline $\mathrm{CD} 34^{+} / \mathrm{KDR}^{+}(\mathrm{xWBC})$ & $113.7 \pm 12.5$ & $335.8 \pm 52.2$ & $P<0.001$ \\
\hline $\mathrm{CD} 4^{+} / \mathrm{CD} 117^{+}(\mathrm{xWBC})$ & $123.0 \pm 21.6$ & $571.3 \pm 86.6$ & $\mathrm{P}<0.001$ \\
\hline $\mathrm{CD} 4^{+} / \mathrm{CD} 133^{+}(\mathrm{xWBC})$ & $67.0 \pm 15.6$ & $275.9 \pm 108.3$ & $P=0.006$ \\
\hline
\end{tabular}


$\mathrm{uL} ; \mathrm{p}<0.001)$. Flow cytometry analysis revealed a significant increase of $\mathrm{CD} 4^{+} / \mathrm{KDR}^{+}$cells (113.7 \pm 13 to $335.8 \pm 52$ cells/uL; $\mathrm{p}<0.001), \mathrm{CD}^{+} 4^{+} / \mathrm{CD} 117^{+}$cells $(123 \pm 22$ to $571.3 \pm 87$ cells $/$ $\mathrm{uL} ; \mathrm{p}<0.001)$ and CD $34^{+} / \mathrm{CD} 133^{+}$cells $(67 \pm 16$ to $275.9 \pm 108$ cells/uL; $\mathrm{p}=0.006)$. Especially, difference of CD34 ${ }^{+} / \mathrm{CD} 117^{+}$ cells correlated significantly with career best record (Pearson correlation coefficient $=0.587 ; p=0.001$ ) and final full course record (Pearson correlation coefficient $=0386 ; \mathrm{p}=0.035$ ). Baseline mean FMD value (16.6 $\pm 12 \%)$ is high compared with general population in other studies. However, there is a significant reduction of FMD after race $(16.6 \pm 12$ to $9.7 \pm 7.2 \%$, p < 0.024).

Conclusion: The results of our study suggest that finishing a marathon race will lead to an upregulation of EPCs in trained healthy marathoners. However, stressful vigorous exercise induced reduction of FMD immediately after marathon. And, the faster runners ran the race, the less $\mathrm{CD} 34^{+} / \mathrm{CD} 117^{+} \mathrm{EPC}$ increased after race.

\section{M-66 \\ Influence of Salt Loading and Potassium Supplement on Short Term Blood Pressure Variability in Salt-Sensitivity Adults}

Mu Jian-Jun, Wang Yang, Ren Ke-Yu, Liu Fu-Qiang, Lian Qiu-Fang, Zheng Shu-Hui

Department of Cardiology, the First Affiliated Hospital of Medical College, Xi'an Jiao Tong University, Xi'an 710061, China

Background: To observe the change of short term blood pressure variability in salt-sensitive normotensive people after sodium-potassium diet, and explore the ralationship between blood pressure variability and salt-sensitivity.

Methods: A baseline survey was carried out in 93 normotensive adults (age $>18$ ) in Mei county, Shaanxi province from April to October, 2004. All subjects were recruitedand sequentially maintained on a protocol with 7 days low salt diet (51.3 mmol/day sodium), 7 days high salt diet (307.7 mmol/ day sodium), and high salt diet with potassium supplementation (307.7 $\mathrm{mmol} /$ day sodium and $60 \mathrm{mmol} /$ day potassium) for another 7 days. We measured blood pressure 3 times on the last day of each stage, and computed the standard deviation and coefficient of variation as indicator of short term blood pressure variability.

Result: The detecting rate of the salt sensitivity was 32.26 percent of all normotensive adults. Compared with non-saltsensitive group, baseline short-term blood pressure variability of salt-sensitive group was larger $(3.70 \pm 1.67$ vs $2.75 \pm 0.91$, $\mathrm{p}<0.01$ ), and especially there was significant difference on systolic pressure $(4.37 \pm 1.13$ vs $2.49 \pm 0.54, \mathrm{p}<0.01)$. Salt restriction and potassium supplementation reduced short term blood pressure variability of salt-sensitivity $(3.24 \pm 1.60$ vs $3.08 \pm 1.18, p>0.05$ and $3.27 \pm 1.38$ vs $3.26 \pm 1.47, p>0.05$ ), while high-salt diet increased blood pressure variability $(3.70 \pm 1.89$ vs $2.97 \pm 1.10, p<0.05)$, Whereas there was no statistically significant difference in non-salt-sensitive group, although the same phenomenon were observed.

Conclusion: High salt intake may play an important role in increasing short-term blood pressure variability, especially salt-sensitivity adults. Salt restriction and potassium supplementation show a protective effect by decreasing short-term blood pressure variability.

\section{M-67 \\ Relationship Between Ambulatory Pulse Pressure Coherent Index and Hypertensive Patients}

Mu Jian-jun, Wang Yang, Ren Ke-yu

Department of Cardiology, the First Affiliated Hospital of Medical College, Xi'an Jiao Tong University, Xi'an 710061, China

Background: To study the predictive value in elderly patients with essential hypertention of the ambulatory pulse pressure coherent index derived from ambulatory blood pressure monitoring on the renal impairment.

Methods: One hundred and four patients (male 56 and female 48) with essential hypertention were divided according to $24 \mathrm{~h}-\mathrm{PP}(\geq 55$ or $<55)$, PPI $(\geq 0.36$ or $<0.36$ ) and AASI $(\geq 0.30$ or $<0.30$ ) respectively to compare the differences of glomerular filtration rate (GFR). We also investigated which is the sensitive predictor in predicting early renal impairment among 24h-PP, PPI and AASI and the relationship between ambulatory pulse pressure coherent index and GFR by drawing the ROC curve line. Above all, the estimated numbers regarding to prognosticate the early injury of kidney were easily obtained by establishing the regression equation.

Results: GFR were significantly lower in 24h-PP $\geq$ $55 \mathrm{mmHg}$, PPI $\geq 0.36$ and AASI $\geq 0.30$ groups than corresponding $24 \mathrm{~h}-\mathrm{PP}<55 \mathrm{mmHg}$, PPI $<0.36$ and AASI $<0.30$ $(\mathrm{P}<0.05)$. By depicting the ROC curve line, the underlying area of $24 \mathrm{~h}-\mathrm{PP} \geq \mathrm{PPI}$ and AASI were $0.636,0.644$ and 0.656 respectively. In addition, there were a inverse correlation in GFR with age $(\mathrm{r}=-0.367, \mathrm{P}=0.000$, course of hypertension $(\mathrm{r}=-0.371, \mathrm{P}=0.000,24 \mathrm{~h}-\mathrm{PP}(\mathrm{r}=-0.254, \mathrm{P}=0.009$, PPI $(\mathrm{r}=$ $-0.262, \mathrm{P}=0.007$ and AASI $(\mathrm{r}=-0.316, \mathrm{P}=0.001$. Moreover, the estimated numbers regarding to prognosticate the early injury of kidney were 57 years old in age, 7.1 years in course of hypertension, $50 \mathrm{mmHg}$ in 24h-PP, 0.36 in PPI and 0.30 in AASI respectively.

Conclusion: With the elevation of $24 \mathrm{~h}-\mathrm{PP}, \mathrm{PPI}$ and AASI, GFR is significantly decreased and the prevalence of renal injury is significantly increased in elderly patients with essential hypertention. It is suggested that $24 \mathrm{~h}-\mathrm{PP} \geq$ $55 \mathrm{mmHg}$, PPI $\geq 0.36$ and AASI $\geq 0.30$ and mild to moderate decrease of GFR may be risk factors for renal injury in elderly patients with Essential Hypertention. Especially AASI is the sensitive pridictor in predicting the early renal impairment, subsequently PPI and 24h-PP. 


\section{M-68 \\ The Relationship Between Pulse Wave Velocity and High Sensitivity C Reactive Protein Text}

Ying Zhang, Jinping Liu, Yunpeng Cheng, Ran Guo,

Wei Song, Ying Liu, Yan Lu, Yinong Jiang

Hospital of Dalian Medical University - Cardiology

Department

Objective: To investigate the relationship between PWV and highly sensitive $\mathrm{C}$ reactive protein (hs-CRP).

Methods and Results: 210 patients were enrolled and divided into three groups: essential hypertension (EH) was 77 cases, hypertension accompanied with impaired glucose tolerance (EH+IGT) was 60 cases, and hypertension accompanied with type 2 diabetes mellitus (EH+T2DM) was 73 cases. Vascular inflammation was measured by highly-sensitive C-reactive protein, and aortic stiffness was measured by carotid femoral pulse wave velocity (cfPWV). After adjusting age and PP, cfPWV, CAVI of EH+T2DM are higher than the other two groups $(\mathrm{P}<0.05)$, and no significant is found between $\mathrm{EH}$ and $\mathrm{EH}+\mathrm{IGR}$; the serum level of hs-CRP in $\mathrm{EH}+\mathrm{T} 2 \mathrm{DM}$ is significantly higher than the EH group $(\mathrm{P}<0.05)$, but not related to EH+IGT group ( $\mathrm{P}>0.05)$. Multiple stepwise regression analysis showed that age, hs-CRP and cfPWV are independently related to each other $(\mathrm{P}<0.05)$.

Conclusions: Hs-CRP is independently correlated with PWV, could be regarded as a probable marker for arterial stiffness.

\section{M-69 \\ Comparison of Aortic Stiffness and Central Blood Pressure Between Patients with Various Hypertensive Conditions: Form BP Behavior Study \\ Sangyeub Lee, Dong-won Kim, Korean Hypertension \\ Research Network investigators \\ Chungbuk national university hospital}

Background: Using ambulatory blood pressure (ABP) monitoring and home blood pressure (HBP) monitoring, we can identify various hypertensive conditions. White coat hypertension is defined as normal ABP or HBP blood pressure when office blood pressure is elevated. Masked hypertension is defined as opposite manner. White coat hypertension (WCH) is considered by some but not all investigators to be a benign condition without increased cardiovascular risk. Pulse wave analysis is a noninvasive method to measure how the reflected pressure wave interacts with central aortic blood pressure (BP) and to assess how it is related to vascular stiffness. Especially the augmentation index (AIx) is an index of wave reflection and a maker of systemic arterial stiffness. It also is associated with risk factors of cardiovascular disease.
Objective: We undertook this study to compare central blood pressure and aortic stiffness as determined by pulse wave analysis in patients with various hypertensive conditions.

Methods: The study is based on the subjects from Blood Pressure Behavior Study, a prospective registry of Korean Hypertension Network, which consist of the 12 cardiovascular centers in Korea. All subjects were naïve hypertensive patients and the radial augmentation index was measured in all patients. White coat hypertension, hypertension, masked hypertension participants were identified after ambulatory blood pressure and office blood pressure monitoring. Using radial artery applanation tonometry, aortic pulse wave analysis was performed.

Augmentation pressure and central aortic blood pressure were measured. Augmentation index (AI) and AI75 were calculated. We measured also epidemiologic, echocardiographic, hemodynamic and laboratory parameters for each hypertensive patients.

Results: We enrolled 515 naïve prehypertensive and hypertensive patients (mean age : $49+-11.7$ y ; $46 \%$ female). Patients with prehypertension, hypertension stage 1,2 were 128(24\%), 182(34\%), 197(37\%) respectively. Patients with normal blood pressure, white coat hypertension, true hypertension, masked hypertension were $40(8 \%), 65(12 \%)$, $314(60 \%)$, masked hypertension (18\%) respectively. In the epidemiologic parameter, body mass index and weight were significantly higher in patients with true hypertension and masked hypertension. In the echocardiographical parameter, there is no significant difference in each group. In the hemodynamic parameter, office blood pressure average, ABP average, maximal morning systolic blood pressure of WCH patients were significantly lower than which of true and masked hypertension. Central blood pressure was significant higher in masked and true hypertension, but results of augmentation index showed no signficant difference between the patients with WCH and MT. But the patients with masked hypertension have relatively low level of augmentation index. Pulse pressure in patients with WCH was higher than with true hypertension. But there was no stastical difference.

Conclusions: These results suggest that as compared to $\mathrm{WCH}$, aortic stiffness in patients with true hypertension and masked hypertension was significantly higher. But about augmentation index, patients with masked hypertension have relatively low level. Further study is needed. 


\section{M-70}

Increased Pulse Wave Velocity and Augmentation Index after Isometric Handgrip Exercise in Patients with Coronary Artery Disease

Seung-Jae Joo, Joon-Hyouk Choi, Song-Yi Kim, Ki-Seok Kim

Jeju National University Hospital

Background: Arterial stiffness of patients with coronary artery disease (CAD), which is expected to be increased due to a generalized atherosclerotic process of human body, may be more evident under the condition of increased cardiac afterload. Isometric handgrip exercise is a simple and easily applicable method to increase cardiac afterload. We investigated the changes of hemodynamic parameters and arterial stiffness indexes after handgrip exercise in patients with CAD.

Methods: Forty two subjects, who underwent coronary angiography (CAG), were enrolled. After CAG, baseline arterial waveforms were traced at the aortic root and external iliac artery using right coronary catheters. Arterial waveforms were recorded at 1, 2 and $3 \mathrm{~min}$ in the aortic root and at $3 \mathrm{~min}$ in the external iliac artery after the isometric handgrip exercise at $30 \sim 40 \%$ of the maximal handgrip power. Augmentation pressure (AP) and augmentation index (AI) were measured at the central aortic waveforms. Pulse wave velocity (PWV) was calculated using the ECG-gated time difference of the upstroke of the arterial waveforms and the distance between the aortic root and the external iliac artery.

Results: Thirty patients had a significant stenosis > $50 \%$ in at least one coronary artery. Their age $(63.4 \pm 8.8$ vs. $62.0 \pm 10.8$ years), sex ratio, and baseline hemodynamic parameters including $\mathrm{AI}$ and PWV were not different from those of subjects without CAD. After handgrip exercise, central systolic blood pressure (BP), diastolic BP, pulse pressure, peripheral systolic BP, and peripheral pulse pressure were increased in all patients. AP and AI inclined significantly from 1 min after exercise only in patients with CAD. PWV also increased significantly only in patients with CAD.

Conclusions: Arterial stiffness indexes at rest were not different between patients with CAD and without CAD. Increased arterial stiffness of patients with CAD became evident only after isometric handgrip exercise.

\section{M-71}

\section{Association between Ambulatory Systolic Blood Pressure at Different Time Window during a Day and Asymptomatic Intracranial Arterial Stenosis Diagnosed with CT Angiography}

\author{
Chaoting Chen ${ }^{1}$, Yan $\mathrm{Li}^{1}$, Jin Zhang ${ }^{1}$, Yan Wang ${ }^{1}$, \\ Huawei Lin ${ }^{2}$, Kemin Chen ${ }^{2}$, Pingjin Gao ${ }^{1}$, Dingliang Zhu ${ }^{1}$ \\ ${ }^{1}$ The State Key Laboratory of Medical Genomics, \\ Department of hypertension, Shanghai Key Laboratory \\ of Vascular Biology, Shanghai Institute of Hypertension; \\ 2 Department of radiology; Ruijin hospital, Shanghai Jiao \\ Tong University School of Medicine, Shanghai, China
}

Background: Ambulatory blood pressure (BP) monitoring provides diurnal BP readings over 24 hours. There is large amount of evidence that 24-hour mean BP, especially systolic, is a strong and independent predictor of cardiovascular disease. However, it remains under investigation that at which time window during 24 hours, BP was more predictive. The present cross-sectional study aimed to compare the size of the associations of cerebrovasulcar atherosclerosis with systolic BPs across 4 time windows of 24 hours.

Methods: In our outpatient clinic and inpatient ward of the Department of Hypertension, 757 consecutive hypertensive patients without history of transient ischemic attack or stroke were enrolled for an ongoing prospective study on intracranial cerebrovascular diseases. Intracranial arterial stenosis (ICAS) was diagnosed with CT angiography. 24-hour ambulatory BP monitoring was performed with SpaceLab monitors. We computed mean values of morning (5 AM to 8 $\mathrm{AM}$ ), daytime (8 $\mathrm{AM}$ to $6 \mathrm{PM}$ ), evening (6 PM to $11 \mathrm{PM}$ ) and nighttime (11 PM to 5 AM) systolic BPs.

Results: ICAS was present in 127 (16.8\%) patients, of whom 64 (50.4\%) patients had moderate-to-severe stenosis $(\geq 50 \%$ ). After multivariate adjustment for age, sex, hypertension duration, body mass index, diabetes mellitus, total and high-density lipoprotein cholesterol, current smoking, alcohol drinking, and antihypertensive treatment, all the four time-window systolic BPs (odds ratio for each $10-\mathrm{mm}$ Hg increment: 1.33 to $1.40, \mathrm{P}<0.001$ ) were significantly

Table 1. (for Abstract M-70)

\begin{tabular}{lllll}
\hline & Baseline & Exercise 1 min & Exercise 2 min & Exercise 3 min \\
\hline Patients with CAD (n=30) & & & \\
$\quad$ AP (mmHg) & $9.3 \pm 5.8$ & $13.8 \pm 7.0^{*}$ & $14.3 \pm 7.7^{*}$ & $14.7 \pm 8.3^{*}$ \\
AI (\%) & $17.7 \pm 9.7$ & $22.1 \pm 9.9^{*}$ & $22.0 \pm 10.5^{*}$ & $22.3 \pm 10.7^{*}$ \\
PWV (m/sec) & $10.03 \pm 1.99$ & & & $11.09 \pm 2.45$ \\
Patients without CAD (n=12) & & & $13.8 \pm 10.3$ & $13.0 \pm 9.3$ \\
AP (mmHg) & $10.0 \pm 8.1$ & $12.9 \pm 10.8$ & $20.4 \pm 13.6$ & $19.9 \pm 1.35$ \\
AI (\%) & $16.5 \pm 12.2$ & $19.3 \pm 14.3$ & & $10.12 \pm 1.79$ \\
PWV (m/sec) & $9.82 \pm 1.10$ & & \\
\hline$* \mathrm{p}<0.05$ vs. baseline & & & \\
\hline
\end{tabular}


associated with the presence of ICAS. However, when the four time-window systolic BPs were put into a single model, only morning systolic BP $(1.29,95 \% \mathrm{CI}, 1.05$ to $1.58, \mathrm{P}=$ 0.02 ) remained significantly associated with ICAS. In further ordinal logistic regression analysis, morning systolic BP was independently and prominently $(1.52,95 \% \mathrm{CI}, 1.18$ to 1.96 , $\mathrm{P}=0.001$ ) associated with the presence of ICAS at 2 vessels or more.

Conclusion: In hypertensive patients, systolic BP in the morning, compared to BP at other time windows, is more closely associated with asymptomatic ICAS. Our findings highlighted the importance of morning systolic BP and should be proved in prospective studies.

\section{M-72 \\ Common Carotid Artery Stiffness Associates with Left Ventricular Function Independently of Aortic Stiffness and Predicts First Hospitalization for Acute Heart Failure \\ Shih-Hsien Sung ${ }^{1,3,4},{ }^{*}$ o-Nan Liao ${ }^{1,3}$, Wen-Chung $\mathrm{Yu}^{1,3}$, Hao-Min Cheng 2,3, Chen-Huan Chen 2,3,4 \\ ${ }^{1}$ Division of Cardiology, Department of Medicine, and ${ }^{2}$ Department of Medical Research and Education, Taipei Veterans General Hospital; ${ }^{3}$ Cardiovascular Research Center, and ${ }^{4}$ Institute of Public Health and Community Medicine Research Center, National Yang-Ming University, Taipei, Taiwan}

Introduction: The role of common carotid arteries (CCA) in the pathogenesis of heart failure remains unclear. The present study investigated the associations of mechanical properties of CCA with left ventricular structure and function, and development of acute heart failure (AHF).

Methods: Outpatients presenting with dyspnea were enrolled for comprehensive noninvasive cardiovascular examinations. LV mass index (LVMi), LV ejection fraction (EF), and ratio of transmitral flow velocity to septal mitral annulus tissue velocity at early diastole (E/E') were measured by echocardiography. CCA mechanical properties, including incremental elastic modulus (Einc), carotid distensibility (CD), $\beta$ stiffness index, and circumferential strain (CS), were assessed by carotid artery ultrasonography. Aortic stiffness was indexed by carotid-femoral pulse wave velocity (cf-PWV) using tonometry.

Results: Among 114 study subjects (aged 63.5 \pm 17.5 years), 60 had normal (NF) and 54 had abnormal LV systolic or diastolic function. Patients with abnormal LV function had significantly greater aortic and CCA stiffness than NF. cf-PWV was significantly associated with EF but not LVMi or E/E', after accounting for age and mean blood pressure. In contrast, all CCA mechanical indices were significantly associated with $\mathrm{EF}$ and E/E', and CD was significantly associated with LVMi, independently of age, mean blood pressure, and cf-PWV. During a mean follow-up of $265 \pm 106$ days, 9 patients incurred AHFS. The highest quartiles of Einc [hazard ratio 6.82, 95\% confidence interval, 1.70-27.35], CS [6.82, 1.70-27.35], and $\beta$ stiffness index [3.91, 1.05-14.57] but not cf-PWV significantly predicted the events.

Conclusions: Mechanical properties of CCA may associate with LV structure and function and predict the first $\mathrm{AHF}$, independently of aortic stiffness.

\section{M-73 \\ The Separate Measurement of the Intima and Media Thickness using High - Frequency Transducer as a Promising Method for Evaluating the Different Effect by Risk Factors of Atherosclerosis

\author{
GeeHee Kim ${ }^{1}$, Ho-Joong Youn', Sang-Hyun IHM² \\ ${ }^{1}$ Department of Internal Medicine, St. Vincent's Hospital, \\ ${ }^{2}$ The Catholic University of Korea, Suwon, Republic of
} Korea}

Objective: Carotid intima-media thickness (CIMT) is associated with several risk factors for atherosclerosis and has been consistently linked to cardiovascular and cerebrovascular disease. This study aimed to investigate the clinical significance of separate measurement of CIMT, which is the sum of the intima (IT) and media thickness (MT), using HighFrequency transducer.

Methods: Among patients who underwent coronary angiography in the Medical Department of St. Mary's Hospital from September 2003 to March 2009, 415 subjects (M:F = 197:218; mean age, 55.1 \pm 10.9 years) who were diagnosed with normal coronary arteries were enrolled in this study. MT and CIMT of the enrolled patients were manually measured using high-frequency ultrasonography.

Results: Age correlated with maximum CIMT ( $\mathrm{r}=0.432$, $\mathrm{p}<0.0001)$, mean CIMT ( $\mathrm{r}=0.488, \mathrm{p}<0.0001)$, and mean MT $(r=0.486, p<0.0001)$. In multivariate analysis, age $(\beta=$ $0.399, \mathrm{p}<0.0001)$, body mass index (BMI) $(\beta=0.077, \mathrm{p}=$ $0.014)$, and hypertension (HTN) ( $\beta=0.085, p=0.010)$ were associated with mean CIMT $(\mathrm{R} 2=0.255)$. Age $(ß=0.442, \mathrm{p}<$ $0.0001)$, BMI $(\beta=0.088, p=0.008)$, and HTN $(\beta=0.107, p=$ $0.002)$ correlated with mean MT $(R 2=0.262)$. There was no significant difference between IT and risk factors.

Conclusions: We noted that separate measurements of IT and MT might be a useful method to evaluate different remodeling processes by multiple risk factors of cardiovascular and cerebrovascular disease in arteries. 


\section{M-74 \\ Relationship Among Branchial Pulse Pressure, 24-h Blood Pressure Variability and Center Augmentation Index in Community-Based Population}

\section{Lifang Liu, Xiongjing Jiang, Jinxiang Xie, Lisheng Liu}

Hypertension center, Dept. of Cardiology, Fuwai Hospital, Peking Union medical college, Beijing 100037, China

Background: To analyze the relationship among branchial pulse pressure (PP), 24-h blood pressure variability (BPV) and center augmentation index (AIx) in communitybased population

Methods: A cross-sectional study was carried out in 164 subjects (aged16-60, means 39.8 \pm 13.1 years). Branchial pulse pressure (SBP-DBP), the mean values of 10 readings was measured at home by trained nurses. BPV was estimated with the SD over 24-h, both awake and sleep, by ambulatory blood pressure monitoring using Spacelabs 90207. Pulse waveforms were recorded from the radial artery by SphygmoCor. Central augmentation index (AIx) was generated using general transfer function.

Results: The systolic blood pressure (SBP) variability was greater than the diastolic blood pressure (DBP) variability using the SD $(\mathrm{P}<0.001)$. SBP variability showed a positive correlation with the branchial pulse pressure and AIx, whereas the SD of the DBP showed a negative correlation with the branchial pulse pressure and AIx. In the multiple regression analysis, after adjustment of age gender and body mass index, the systolic BPV measure maintained their associations with the branchial pulse pressure and the AIx.

Conclusion: BPV in community-based population is associated with arterial stiffness as evaluated with brachial pulse pressure and the AIx. The assessment of BPV (24-h) may be useful for assessment of arterial stiffness.

\section{M-75 \\ Effect of Carotid Baroreceptor Activation on the Dynamic Relation Between Arterial Pressure and Pulse Transit Time in the Spontaneously Hypertensive Rat}

Zahra Kouchaki, George Lindesay, Mark Butlin, Alberto P. Avolio

Australian School of Advanced Medicine, Macquarie University, Sydney, Australia

Baroreceptor activation (BRA) is used for treatment of resistant hypertension, with possible additional effect on the vasculature beyond the associated decrease in arterialpressure. This study assesses the effects of carotid BRA on the dynamic relationship between mean arterial pressure (MAP) and pulse transit time (PTT).
Table 1. (for Abstract M-75)

\begin{tabular}{llll}
\hline $\begin{array}{l}\text { Frequency } \\
\text { range }(\mathrm{Hz})\end{array}$ & $\begin{array}{l}\text { Magnitude }\left(^{*}\right) \\
\text { Stim-ON }\end{array}$ & $\begin{array}{l}\text { Magnitude }\left(^{*}\right) \\
\text { Stim-OFF }\end{array}$ & $\mathrm{P}\left({ }^{* *}\right)$ \\
\hline $0-1$ & $7.3 \pm 3.8$ & $3.6 \pm 1.4$ & 0.042 \\
$1-2$ & $12.2 \pm 5.1$ & $4.6 \pm 1.3$ & 0.007 \\
$2-3$ & $12.3 \pm 5.1$ & $6.9 \pm 2.8$ & 0.045 \\
\hline
\end{tabular}

(*) Units: $10-5 \mathrm{~ms} / \mathrm{mmHg}$; (**) paired t-test.

Anaesthetised, spontaneously hypertensive rats (SHR) ( $\mathrm{n}=3,13$ weeks) were implanted with a field stimulation electrodes surrounding the common carotid artery, immediately proximal to the carotid bifurcation. MAP and aortic PTT, a measure of vessel stiffness, were measured using two pressure sensors located in the proximal and distal descending aorta. Field stimulation was conducted at 1000 $\mathrm{Hz}$ with a pulse width of $0.53 \mathrm{~ms}$. Threshold voltage (TV) was classified as the voltage which produced a $5 \mathrm{mmHg}$ drop in MAP. Measurements were then taken at 2 (x2TV), 3 (x3TV), and 3.5 (x3.5TV) times TV with a stimulationperiod of 20 seconds. The frequency transfer function (TF) between MAP and PTT was determined as the ratio of the magnitudes of the frequency components of PTT and MAP from beat-to-beat values of the time series of PTT (ms) and MAP (mmHg) calculated during (Stim-ON) and following stimulation (Stim-OFF) in the frequency ranges of $0-1,1-2$, and $2-3 \mathrm{~Hz}$. The x2TV did not show any significant difference between Stim-ON and Stim-OFF. However, x3TV and 3.5TV showed consistently higher and similar TF magnitudes for Stim-ON in all rats and all stimulations. Table shows the pooled results for x3TV and x3.5TV $(n=6)$. The spectra for the TF of Stim-ON and Stim-OFF spectra are significantly different with the greatest different being at $1-2 \mathrm{~Hz}$

Field stimulation of carotid baroreceptors in the SHR reduces MAP and also changes the dynamic characteristics of the relationships between PTT and MPA. Future analysis will be aimed to determine whether the effect is mediated by the effect of BRA or due to concomitant changes in MAP.

\section{M-76 \\ Aortic Stiffness Is Not Associated with Acute Elevation of Circulating Interleukin- 6 in a Novel Rodent Model of Inflammation}

Mark Butlin, Ying-Yi Liu, Alberto P. Avolio

Australian School of Advanced Medicine, Macquarie University, Sydney, Australia

Acute inflammation is associated with endothelial dysfunction. However, understanding of the mechanism is limited. This study investigated the effect of acute inflammation on aortic stiffness in the rat using a novel model of induced inflammation. Rats (Sprague-Dawley) were injected 
Table 1. (for Abstract M-76)

\begin{tabular}{|c|c|c|c|}
\hline & Control & $\begin{array}{l}\text { TNF- } \alpha \\
\text { injected }\end{array}$ & $\mathrm{p}$ \\
\hline Interleukin-6 (pg/ml) & $488 \pm 13$ & $1258 \pm 159$ & $<0.01$ \\
\hline c-reactive protein (ng/ml) & $4.7 \pm 1.2$ & $2.1 \pm 0.2$ & $<0.05$ \\
\hline e-selectin (pg/ml) & $319 \pm 74$ & $234 \pm 28$ & NS \\
\hline $\begin{array}{l}\text { PWV (m/s at MAP of } \\
120 \mathrm{mmHg})\end{array}$ & $4.4 \pm 0.3$ & $4.4 \pm 0.3$ & NS \\
\hline $\begin{array}{l}\text { Breaking stress, thoracic } \\
(\mathrm{N} / \mathrm{mm} / \mathrm{mg})\end{array}$ & $0.19 \pm 0.07$ & $0.14 \pm 0.11$ & NS \\
\hline $\begin{array}{l}\text { Breaking stress, abdominal } \\
(\mathrm{N} / \mathrm{mm} / \mathrm{mg})\end{array}$ & $0.41 \pm 0.29$ & $0.32 \pm 0.14$ & NS \\
\hline $\begin{array}{l}\text { Breaking strain, } \\
\text { thoracic }(\%)\end{array}$ & $106 \pm 26$ & $138 \pm 31$ & NS \\
\hline $\begin{array}{l}\text { Breaking strain, } \\
\text { abdominal (\%) }\end{array}$ & $98 \pm 20$ & $133 \pm 32$ & NS \\
\hline
\end{tabular}

with recombinant tumour necrosis factor $\alpha$ (TNF- $\alpha, 8 \mu \mathrm{g} /$ $\mathrm{kg}$ viatail-vein, $\mathrm{n}=5$ ) or vehicle $(\mathrm{n}=5)$ at 11 weeks of age. At 12 weeks, rats were anaesthetisedand theaortic pulse wave velocity (PWV), a measure of stiffness, measured in the descending aorta. PWV was assessed across a full physiological range of mean arterial pressure (MAP) byalternate infusion of sodium nitroprusside and phenylephrine (both $50 \mu \mathrm{g} / \mathrm{kg} / \mathrm{min}$ ). Blood plasma was analysed for circulating levels of interleukin-6, c-reactive protein (CRP) and e-selectin. Thoracic and abdominal aortic rings were dissected and submitted to tensile testing.

Circulating levels of interleukin-6 were raised, CRP lowered, with no change in e-selectin (Table). PWV was not different at any MAP (120 mmHg shown in Table). There was a trend toward greater aortic wall stiffness with TNF- $\alpha$ injection as measured by tensile testing, but this did not result in significant differences in aortic breaking stress or strain. Recombinant TNF- $\alpha$ caused a 2.6-fold increase in interleukin- 6 and a trend toward greater aortic wall material stiffness but no observable acute changes in in-vivo functional aortic stiffness.

Functional changes in aortic stiffness may require higher levels of circulating inflammatory markers to induce changes in endothelial response, and longer exposure to evoke greater changes in structural wall components.

\section{M-77 \\ Effect of Diameter Reduction on Aortic Wall Shear Stresses: A Pilot Computational Fluid Dynamic Study of a Model of Aortic Wrap}

Francesca Giudici, Michael O'Rourke, Yi Qian, Azadeh Farnoush, Alberto Avolio

ASAM, Macquarie University, Sydney, Australia

Age-related stiffening of the ascending aorta (AA) is a major determinant of isolated systolic hypertension. The aortic wrap $(\mathrm{AW})$ is a technique aimed at functionally destiffening the AA by wrapping it with a more distensible elastic material and reducing the diameter; the pulsatile load is transferred from the AA to the AW, thus increasing distensibility of the vascular segment. The diameter reduction, though necessary for wall unloading, can create flow disturbances and increase arterial wall shear stresses (WSS). This study aimed at a preliminary computational fluid dynamic (CFD) investigation of the effects of AWon aortic WSS.

A 3D model of a straight tube with a diameter comparable to that of an AA was used to create 16 wrapped geometrical configurations (4 different wrap lengths of 5, 10 15, $20 \mathrm{~cm}$ and 4 different wrap diameters: $10 \%, 20 \%, 30 \%, 40 \%$ reduction of a nominal diameterof $22 \mathrm{~mm}$ ). A CFD investigation was performed (ANSYS Fluent), applying a steady flow velocity $(0.22 \mathrm{~m} / \mathrm{s})$ at the inlet and imposing no-slip and traction-free boundary conditions on the walls and at the outlet respectively. The fluid was considered an incompressible Newtonian fluid with blood density and viscosity, and the arterial wall deformation was neglected. The narrowing due to the wrap diameter reduction increases the maximum WSS values (range 1.01 and 3.1 fold), which are located along the distal edge of the AW; it increasesthe maximum velocity (range 1.13 and 2.74 fold); it amplifies the pressure drop along the model (range 1.43 and 13.22 fold) and it creates areas of stagnating flow and very lowWSS (range 0.001 and 0.53 times the unwrapped value) in the end of the tube distal to the AW. The maximum WSS increase, pressure drop amplification, and maximum velocity change are all dependent on the area of wrapped surface and the amount of diameter reduction.

This preliminary CFD study shows that the diameter reduction associated with AW leads todistal increased WSS and disturbed flow. To minimize these drawbacks, a compromise between unloading efficacy and lumen reduction has to be pursued. Further studies considering a time-varying velocity input profile, realistic geometries, and deformable walls will improve the characterization of AW effects on $\mathrm{AA}$ fluid dynamics. 